Internet Engineering Task Force (IETF)

Request for Comments: 6956

Category: Standards Track

ISSN : $2070-1721$
W. Wang

Zhejiang Gongshang University

E. Haleplidis

University of Patras

K. Ogawa

NTT Corporation

C. Li

Hangzhou DPtech

J. Halpern

Ericsson

June 2013

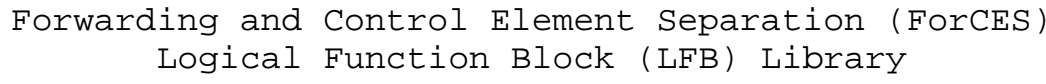

Abstract

This document defines basic classes of Logical Function Blocks (LFBs) used in Forwarding and Control Element Separation (ForCES). The basic LFB classes are defined according to the ForCES Forwarding Element (FE) model and ForCES protocol specifications; they are scoped to meet requirements of typical router functions and are considered the basic LFB library for ForCES. The library includes the descriptions of the LFBs and the XML definitions.

Status of This Memo

This is an Internet Standards Track document.

This document is a product of the Internet Engineering Task Force (IETF). It represents the consensus of the IETF community. It has received public review and has been approved for publication by the Internet Engineering Steering Group (IESG). Further information on Internet standards is available in section 2 of RFC 5741.

Information about the current status of this document, any errata, and how to provide feedback on it may be obtained at http://www.rfc-editor.org/info/rfc6956. 
Copyright Notice

Copyright (c) 2013 IETF Trust and the persons identified as the document authors. All rights reserved.

This document is subject to BCP 78 and the IETF Trust's Legal Provisions Relating to IETF Documents

(http://trustee.ietf.org/license-info) in effect on the date of publication of this document. Please review these documents carefully, as they describe your rights and restrictions with respect to this document. Code Components extracted from this document must include Simplified BSD License text as described in Section 4.e of the Trust Legal Provisions and are provided without warranty as described in the Simplified BSD License.

Table of Contents

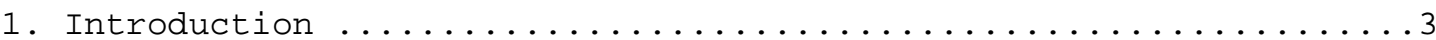

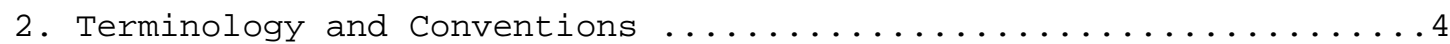

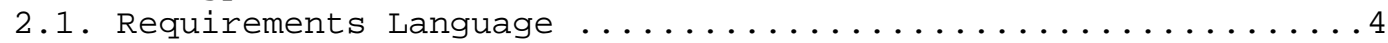

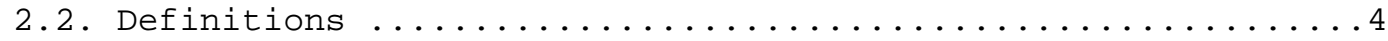

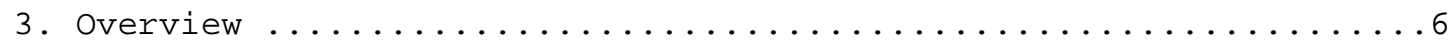

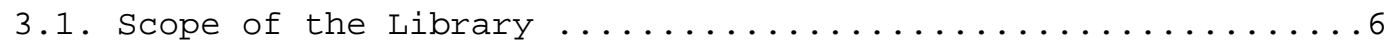

3.2. Overview of LFB Classes in the Library ................

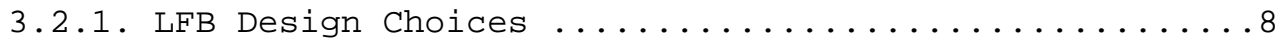

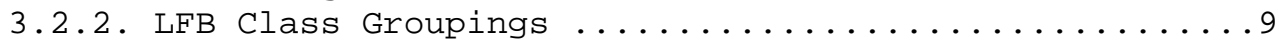

3.2.3. Sample LFB Class Application .................. 10

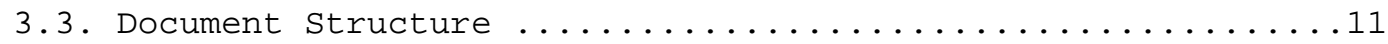

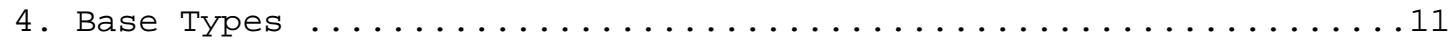

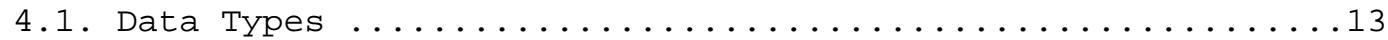

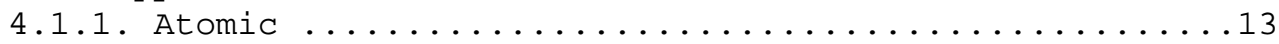

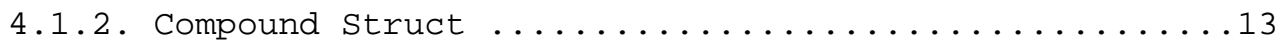

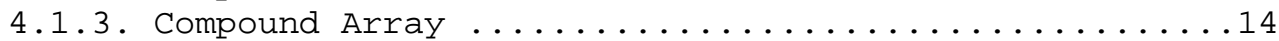

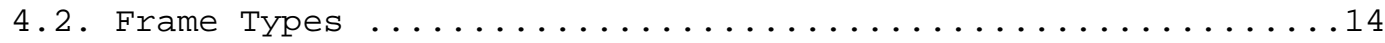

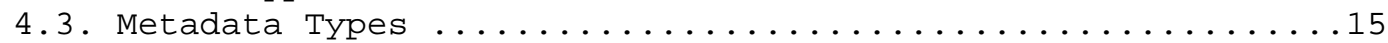

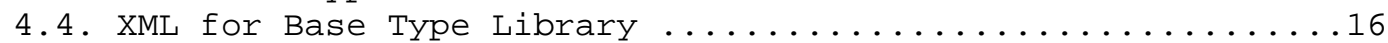

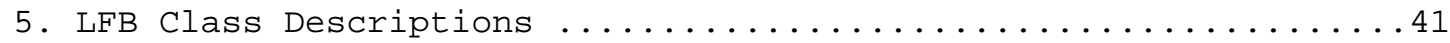

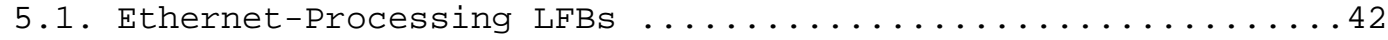

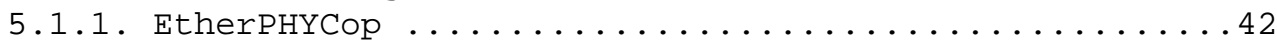

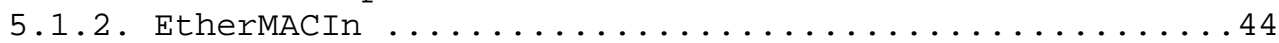

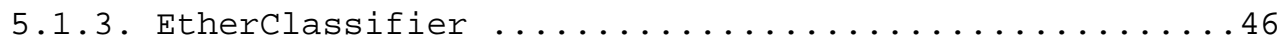

5.1 .4 . EtherEncap .........................

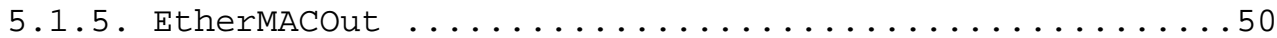

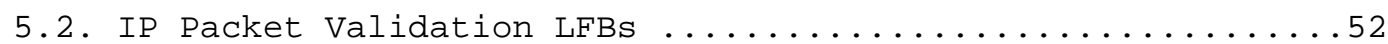

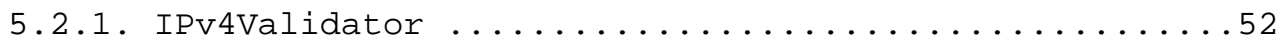

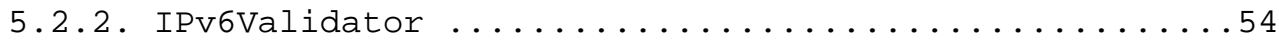




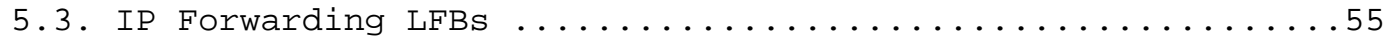

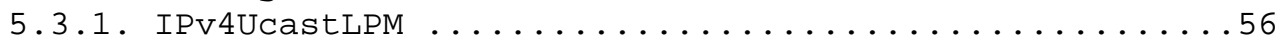

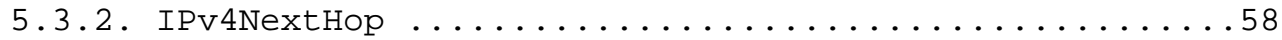

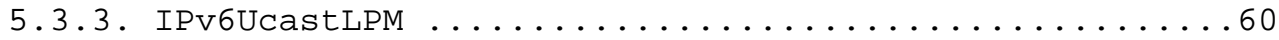

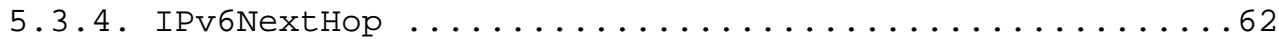

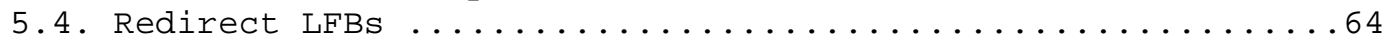

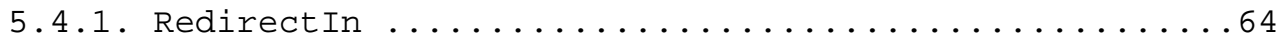

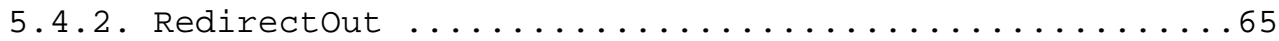

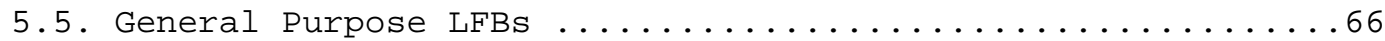

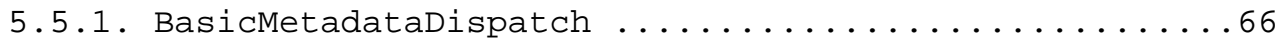

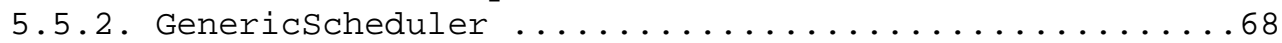

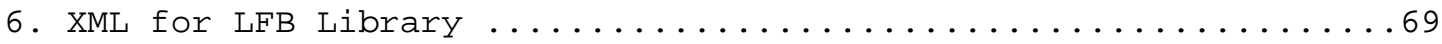

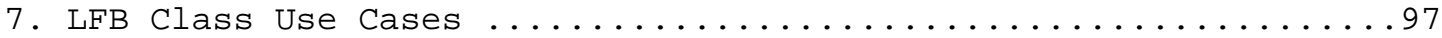

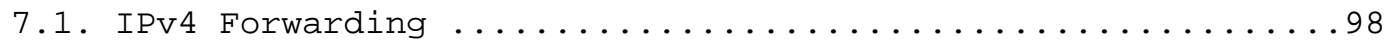

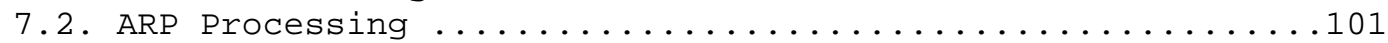

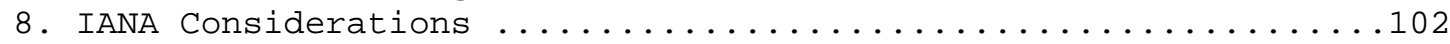

8.1. LFB Class Names and LFB Class Identifiers ............103

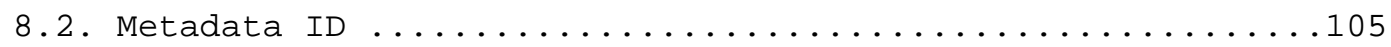

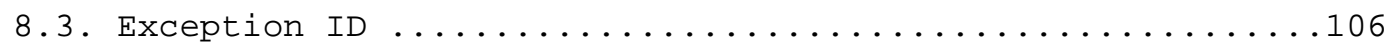

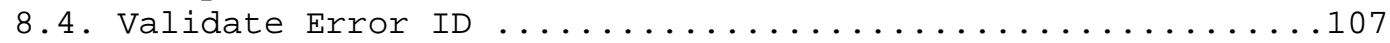

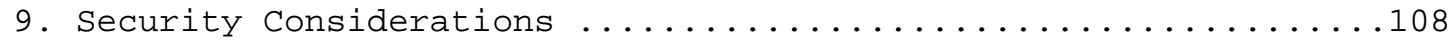

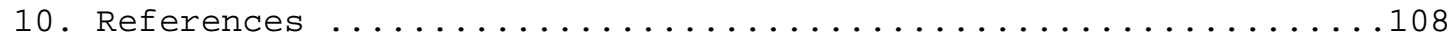

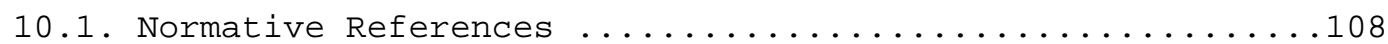

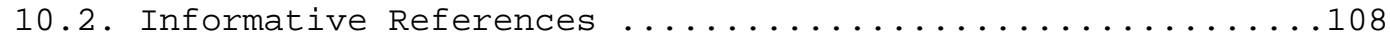

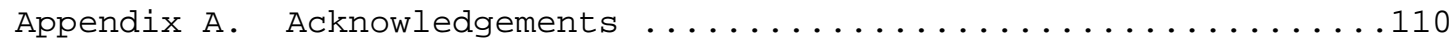

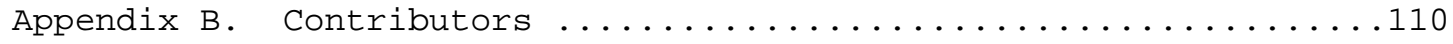

1. Introduction

[RFC3746] specifies the Forwarding and Control Element Separation

(ForCES) framework. In the framework, Control Elements (CES)

configure and manage one or more separate Forwarding Elements (FEs) within a Network Element (NE) by use of a ForCES protocol. [RFC5810] specifies the ForCES protocol. [RFC5812] specifies the Forwarding Element (FE) model. In the model, resources in FEs are described by classes of Logical Function Blocks (LFBs). The FE model defines the structure and abstract semantics of LFBs and provides XML schema for the definitions of LFBs.

This document conforms to the specifications of the FE model [RFC5812] and specifies detailed definitions of classes of LFBs, including detailed XML definitions of LFBs. These LFBs form a base LFB library for ForCES. LFBs in the base library are expected to be combined to form an LFB topology for a typical router to implement IP forwarding. It should be emphasized that an LFB is an abstraction of functions rather than implementation details. The purpose of the LFB definitions is to represent functions so as to provide interoperability between separate CEs and FEs. 
More LFB classes with more functions may be developed in the future and documented by the IETF. Vendors may also develop proprietary LFB classes as described in the FE model [RFC5812].

\section{Terminology and Conventions}

\subsection{Requirements Language}

The key words "MUST", "MUST NOT", "REQUIRED", "SHALL", "SHALL NOT", "SHOULD", "SHOULD NOT", "RECOMMENDED", "MAY", and "OPTIONAL" in this document are to be interpreted as described in [RFC2119].

\subsection{Definitions}

This document follows the terminology defined by the ForCES protocol in [RFC5810] and by the ForCES FE model in [RFC5812]. The definitions below are repeated for clarity.

Control Element (CE) - A logical entity that implements the ForCES protocol and uses it to instruct one or more FEs on how to process packets. CEs handle functionality such as the execution of control and signaling protocols.

Forwarding Element (FE) - A logical entity that implements the ForCES protocol. FEs use the underlying hardware to provide perpacket processing and handling as directed/controlled by one or more CEs via the ForCES protocol.

ForCES Network Element (NE) - An entity composed of one or more $\mathrm{CES}$ and one or more FEs. To entities outside an NE, the $\mathrm{NE}$ represents a single point of management. Similarly, an NE usually hides its internal organization from external entities.

Logical Function Block (LFB) - The basic building block that is operated on by the ForCES protocol. The LFB is a well-defined, logically separable functional block that resides in an $\mathrm{FE}$ and is controlled by the CE via the ForCES protocol. The LFB may reside at the FE's data path and process packets or may be purely an FE control or configuration entity that is operated on by the CE. Note that the LFB is a functionally accurate abstraction of the FE's processing capabilities but not a hardware-accurate representation of the $\mathrm{FE}$ implementation.

FE Model - The FE model is designed to model the logical processing functions of an FE, which is defined by the ForCES FE model document [RFC5812]. The FE model proposed in this document includes three components: the LFB modeling of individual Logical Functional Blocks (LFB model), the logical interconnection between 
LFBs (LFB topology), and the FE-level attributes, including FE capabilities. The FE model provides the basis to define the information elements exchanged between the $\mathrm{CE}$ and the $\mathrm{FE}$ in the ForCES protocol [RFC5810].

FE Topology - A representation of how the multiple FEs within a single $\mathrm{NE}$ are interconnected. Sometimes this is called inter-FE topology, to be distinguished from intra-FE topology (i.e., LFB topology).

LFB Class and LFB Instance - LFBs are categorized by LFB classes. An LFB instance represents an LFB class (or type) existence. There may be multiple instances of the same LFB class (or type) in an FE. An LFB class is represented by an LFB class ID, and an LFB instance is represented by an LFB instance ID. As a result, an LFB class ID associated with an LFB instance ID uniquely specifies an LFB existence.

LFB Metadata - Metadata is used to communicate per-packet state from one LFB to another but is not sent across the network. The FE model defines how such metadata is identified, produced, and consumed by the LFBS. It defines the functionality but not how metadata is encoded within an implementation.

LFB Component - Operational parameters of the LFBs that must be visible to the CEs are conceptualized in the FE model as the LFB components. The LFB components include, for example, flags, single parameter arguments, complex arguments, and tables that the $\mathrm{CE}$ can read and/or write via the ForCES protocol (see below).

LFB Topology - Representation of how the LFB instances are logically interconnected and placed along the data path within one FE. Sometimes it is also called intra-FE topology, to be distinguished from inter-FE topology.

Data Path - A conceptual path taken by packets within the forwarding plane inside an FE. Note that more than one data path can exist within an FE.

ForCES Protocol - While there may be multiple protocols used within the overall ForCES architecture, the term "ForCES protocol" and "protocol" refer to the Fp reference points in the ForCES framework in [RFC3746]. This protocol does not apply to CE-to-CE communication, FE-to-FE communication, or to communication between $\mathrm{FE}$ and $\mathrm{CE}$ managers. Basically, the ForCES protocol works in a master-slave mode in which FEs are slaves and CEs are masters. 
Physical Port - A port refers to a physical media input port or output port of an FE. A physical port is usually assigned with a physical port ID, abbreviated with a PHYPortID. This document mainly deals with physical ports with Ethernet media.

Logical Port - A conceptually virtual port at the data link layer (L2) or network layer (L3). A logical port is usually assigned with a logical port ID, abbreviated with a LogicalPortID. The logical ports can be further categorized with an L2 logical port or an L3 logical port. An L2 logical port can be assigned with an L2 logical port ID, abbreviated with an L2PortID. An L3 logical port can be assigned with an L3 logical port ID, abbreviated with an L3PortID. MAC-layer VLAN ports belong to logical ports, and they belong to L2 logical ports.

LFB Port - The connection points where one LFB can be connected to another within an FE. As described in [RFC5812], the CE can connect LFBs together by establishing connections between an output port of one LFB instance and an input port of another LFB instance. Also see section 3.2 of [RFC5812] for more details.

Singleton Port - A named input or output port of an LFB. This port is referred to by a name. When the context is clear, the term "singleton" by itself is used to refer to a singleton port.

Group Port - A named collection of input or output ports of an LFB. A group port is referred to by a name. A group port consists of a number of port instances, which are referred to by a combination of a name and an index.

LFB Class Library - The LFB class library is a set of LFB classes that has been identified as the most common functions found in most FEs and hence should be defined first by the ForCES Working Group. The LFB class library is defined by this document.

3. Overview

\subsection{Scope of the Library}

It is intended that the LFB classes described in this document are designed to provide the functions of a typical router. [RFC1812] specifies that a typical router is expected to provide functions to: 
(1) Interface to packet networks and implement the functions required by that network. These functions typically include:

* Encapsulating and decapsulating the IP datagrams with the connected network framing (e.g., an Ethernet header and checksum),

* Sending and receiving IP datagrams up to the maximum size supported by that network (this size is the network's Maximum Transmission Unit or MTU),

* Translating the IP destination address into an appropriate network-level address for the connected network (e.g., an Ethernet hardware address), if needed, and

* Responding to network flow control and error indications, if any •

(2) Conform to specific Internet protocols including the Internet Protocol (IPv4 and/or IPv6), Internet Control Message Protocol (ICMP), and others as necessary.

(3) Receive and forward Internet datagrams. Important issues in this process are buffer management, congestion control, and fairness.

* Recognize error conditions and generate ICMP error and information messages as required.

* Drop datagrams whose time-to-live fields have reached zero.

* Fragment datagrams when necessary to fit into the MTU of the next link or interface.

(4) Choose a next-hop destination for each IP datagram, based on the information in its routing database.

(5) Usually support an interior gateway protocol (IGP) to carry out distributed routing and reachability algorithms with the other routers in the same autonomous system. In addition, some routers will need to support an exterior gateway protocol to exchange topological information with other autonomous systems. For all routers, it is essential to provide the ability to manage static routing items.

(6) Provide network management and system support facilities, including loading, debugging, status reporting, statistics query, exception reporting, and control. 
The classical IP router utilizing the ForCES framework constitutes a CE running some controlling IGP and/or EGP function or static route setup and FEs implemented by use of Logical Function Blocks (LFBs) conforming to the FE model [RFC5812] specification. The $\mathrm{CE}$, in conformance to the ForCES protocol [RFC5810] and the FE model [RFC5812] specifications, instructs the LFBs on the FE how to treat received/sent packets.

Packets in an IP router are received and transmitted on physical media typically referred to as "ports". Different physical media will have different ways for encapsulating outgoing frames and decapsulating incoming frames. The different physical media will also have different attributes that influence its behavior and how frames get encapsulated or decapsulated. This document will only deal with Ethernet physical media. Future documents may deal with other types of media. This document will also interchangeably refer to a port as an abstraction that constitutes a physical layer (PHY) and a Media Access Control (MAC) layer, as described by LFBs like EtherPHYCop, EtherMACIn, and EtherMACOut.

IP packets emanating from port LFBs are then processed by a validation LFB before being further forwarded to the next LFB. After the validation process, the packet is passed to an LFB where an IP forwarding decision is made. In the IP Forwarding LFBs, a Longest Prefix Match LFB is used to look up the destination information in a packet and select a next-hop index for sending the packet onward. A next-hop LFB uses the next-hop index metadata to apply the proper headers to the IP packets and direct them to the proper egress. Note that in the process of IP packet processing, in this document, we are adhering to the weak-host model [RFC1122] since that is the most usable model for a packet processing a Network Element.

\subsection{Overview of LFB Classes in the Library}

It is critical to classify functional requirements into various classes of LFBs and construct a typical but also flexible enough base LFB library for various IP forwarding equipments.

\subsubsection{LFB Design Choices}

A few design principles were factored into choosing what the base LFBs look like:

- If a function can be designed by either one LFB or two or more LFBs with the same cost, the choice is to go with two or more LFBs so as to provide more flexibility for implementers. 
- An LFB should take advantage of its independence as much as possible and have minimal coupling with other LFBs. The coupling may be from LFB attributes definitions as well as physical implementations.

- Unless there is a clear difference in functionality, similar packet processing in the base LFB library should not be represented simultaneously as two or more LFBs. For instance, it should not be simultaneously defined with two different LFBs for the same next-hop processing. Otherwise, it may add extra burden on implementation to achieve interoperability.

\subsubsection{LFB Class Groupings}

This document defines groups of LFBs for typical router function requirements:

(1) A group of Ethernet-processing LFBs are defined to abstract the packet processing for Ethernet as the port media type. As Ethernet is the most popular media type with rich processing features, Ethernet media processing LFBs were a natural choice. Definitions for processing of other port media types like Packet over SONET (POS) or Asynchronous Transfer Mode (ATM) may be incorporated in the library in future versions of this document or in a separate document. The following LFBs are defined for Ethernet processing:

* EtherPHYCop (Section 5.1.1)

* EtherMACIn (Section 5.1.2)

* Etherclassifier (Section 5.1.3)

* EtherEncap (Section 5.1.4)

* EthermACOut (Section 5.1.5)

(2) A group of LFBs are defined for IP packet validation process. The following LFBs are defined for IP validation processing:

* IPv4Validator (Section 5.2.1)

* IPv6Validator (Section 5.2.2)

(3) A group of LFBs are defined to abstract IP forwarding process. The following LFBs are defined for IP forwarding processing:

* IPv4Ucastlpm (Section 5.3.1) 
* IPv4Nexthop (Section 5.3.2)

* IPv6Ucastlpm (Section 5.3.3)

* IPv6NextHop (Section 5.3.4)

(4) A group of LFBs are defined to abstract the process for redirect operation, i.e., data packet transmission between $\mathrm{CE}$ and FEs. The following LFBs are defined for redirect processing:

* RedirectIn (Section 5.4.1)

* Redirectout (Section 5.4.2)

(5) A group of LFBs are defined for abstracting some general purpose packet processing. These processing processes are usually general to many processing locations in an FE LFB topology. The following LFBs are defined for redirect processing:

* BasicMetadataDispatch (Section 5.5.1)

* GenericScheduler (Section 5.5.2)

\subsubsection{Sample LFB Class Application}

Although Section 7 will present use cases for the LFBs defined in this document, this section shows a simple sample LFB class application in advance so that readers can get a quick overlook of the LFB classes with the usage.

Figure 1 shows a simple LFB processing path for Ethernet packets entered from Ethernet physical ports.

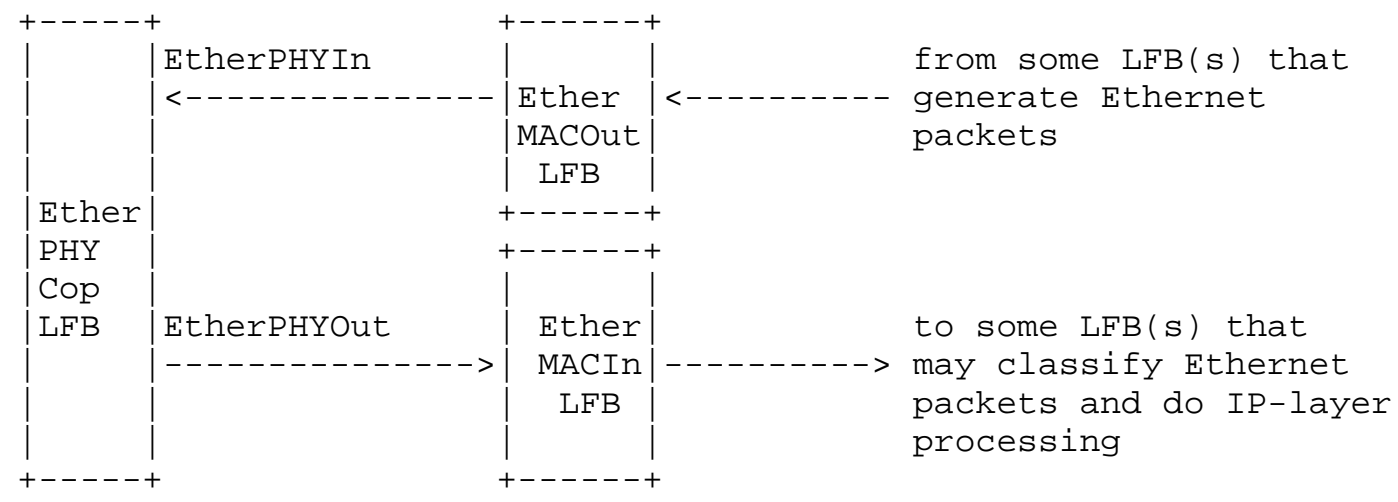

Figure 1: A Simple Sample LFB Use Case 
In the figure, Ethernet packets from outer networks enter via the EtherPHYCop LFB (Section 5.1.1), which describes Ethernet copper interface properties (like the link speed) at the physical layer. After physical-layer processing, Ethernet packets are delivered to the EtherMACIn LFB (Section 5.1.2) to describe its MAC-layer processing functions (like locality check). The packets after the EtherMACIn LFB may require further processing to implement various functions (like IP-layer forwarding); therefore, some LFBs may follow the EtherMACIn LFB in topology to describe followed processing functions.

Meanwhile, packets generated by some LFB(s) may need to be submitted to outer physical networks. The process is described in the figure by an EtherMACOut LFB (Section 5.1.5) at the MAC layer and the EtherPHYCop LFB at the physical layer.

\subsection{Document structure}

Base type definitions, including data types, packet frame types, and metadata types, are presented in advance for definitions of various LFB classes. Section 4 ("Base Types") provides a description on the base types used by this LFB library. To enable extensive use of these base types by other LFB class definitions, the base type definitions are provided as a separate library.

Within every group of LFB classes, a set of LFBs are defined for individual function purposes. Section 5 ("LFB Class Descriptions") provides text descriptions on the individual LFBs. Note that for a complete definition of an LFB, a text description and an XML definition are required.

LFB classes are finally defined by XML with specifications and schema defined in the ForCES FE model [RFC5812]. Section 6 ("XML for LFB Library") provides the complete XML definitions of the base LFB classes library.

Section 7 provides several use cases on how some typical router functions can be implemented using the base LFB library defined in this document.

4. Base Types

The FE model [RFC5812] has specified predefined (built-in) atomic data types: char, uchar, int16, uint16, int32, uint32, int64, uint64, string[N], string, byte[N], boolean, octetstring[N], floatl6, float 32 , and float 64 . 
Note that, unlike the Simple Network Management Protocol (SNMP) information model, called the structure of Management Information (SMI) [RFC2578], the FE model has not defined specific atomic data types for counting purposes. This document also does not define specific counter types. To describe LFB elements for packet statistics, which actually requires counters on packets, an unsigned integer, like an uint32 or an uint64, is adopted. This document states that any LFB element defined for counting purposes is specified to monotonically increase until it reaches a maximum value, when it wraps around and starts increasing again from zero. This document also states that how the unsigned integer element might be maintained to cope with issues like counter discontinuities when a counter wraps or is reset for any reason is an implementation's issue. If a $\mathrm{CE}$ is expected to understand more meanings of the counter element than stated above, a private definition on the element between the $\mathrm{CE}$ and $\mathrm{FE}$ may be required.

Based on the atomic data types and with the use of type definition elements in the FE model XML schema, new data types, packet frame types, and metadata types can be defined.

To define a base LFB library for typical router functions, a set of base data types, frame types, and metadata types should be defined. This section provides a brief description of the base types and a full XML definition of them as well.

The base type XML definitions are provided with a separate XML library file named "BaseTypeLibrary". Users can refer to this library by the statement:

<load library="BaseTypeLibrary" location="..."/> 


\subsection{Data Types}

Data types defined in the base type library are categorized by the following types: atomic, compound struct, and compound array.

4.1.1. Atomic

The following data types are defined as atomic data types and put in the base type library:

$\begin{array}{ll}\text { Data Type Name } & \text { Brief Description } \\ --------- & ----------- \\ \text { IPv4Addr } & \text { IPv4 address } \\ \text { IPv6Addr } & \text { IPv6 address } \\ \text { IEEEMAC } & \text { IEEE MAC address } \\ \text { LANSpeedType } & \text { LAN speed by value types } \\ \text { DuplexType } & \text { Duplex types } \\ \text { PortStatusType } & \text { The possible types of port status, used for } \\ & \text { both administrative and operative status } \\ \text { VlanIDType } & \text { The type of VLAN ID } \\ \text { VlanPriorityType } & \text { The type of VLAN priority } \\ \text { SchdDisciplineType } & \text { Scheduling discipline type }\end{array}$

4.1.2. Compound Struct

The following compound struct types are defined in the base type library:

Data Type Name
EtherDispatchEntryType
VlanInputTableEntryType
EncapTableEntryType
MACInStatsType
MACOutStatsType
EtherClassifyStatsType
IPv4PrefixInfoType
IPv6PrefixInfoType
IPv4NextHopInfoType
IPv6NextHopInfoType
IPv4ValidatorStatsType
IPv6ValidatorStatsType
IPv4UcastLPMStatsType
IPv6UcastLPMStatsType
QueueStatsType
MetadataDispatchType

Wang, et al.

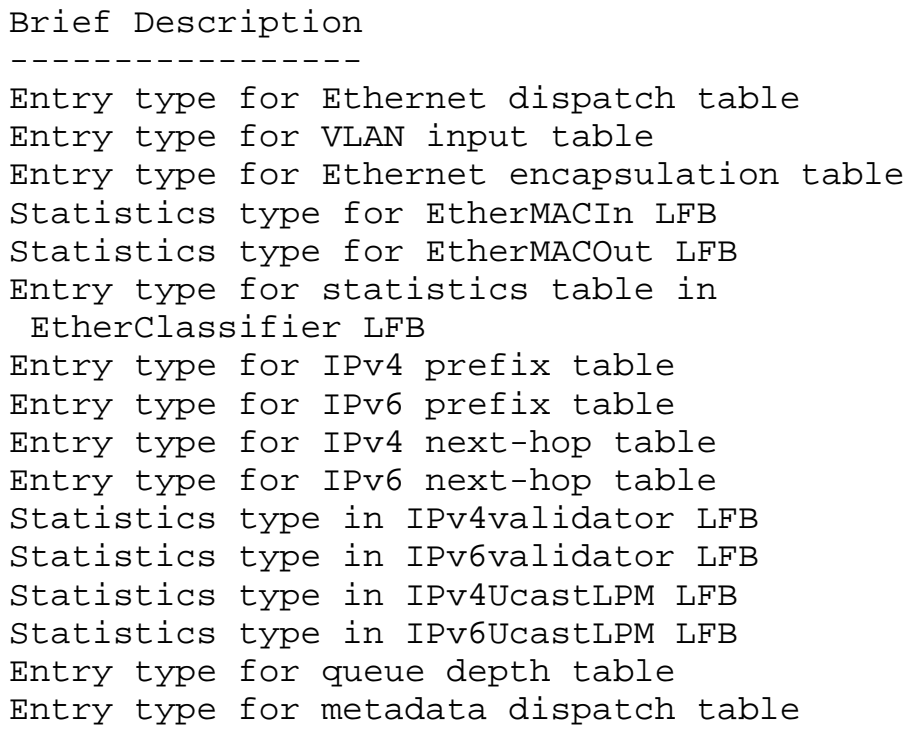

Standards Track

[Page 13] 


\subsubsection{Compound Array}

Compound array types are mostly created based on compound struct types for LFB table components. The following compound array types are defined in this base type library:

$\begin{array}{ll}\text { Data Type Name } & \text { Brief Description } \\ \text { EtherClassifyStatsTableType } & \text { Type for Ethernet classifier statistics } \\ & \text { information table } \\ \text { EtherDispatchTableType } & \text { Type for Ethernet dispatch table } \\ \text { VlanInputTableType } & \text { Type for VLAN input table } \\ \text { EncapTableType } & \text { Type for Ethernet encapsulation table } \\ \text { IPv4PrefixTableType } & \text { Type for IPv4 prefix table } \\ \text { IPv6PrefixTableType } & \text { Type for IPv6 prefix table } \\ \text { IPv4NextHopTableType } & \text { Type for IPv4 next-hop table } \\ \text { IPv6NextHopTableType } & \text { Type for IPv6 next-hop table } \\ \text { MetadataDispatchTableType } & \text { Type for Metadata dispatch table } \\ \text { QueueStatsTableType } & \text { Type for Queue depth table }\end{array}$

\subsection{Frame Types}

According to the FE model [RFC5812], frame types are used in LFB definitions to define packet frame types that an LFB expects at its input port and that the LFB emits at its output port. The <frameDef> element in the FE model is used to define a new frame type.

The following frame types are defined in the base type library:

Frame Name
EthernetII
ARP
IPv4
IPv6
IPv4Unicast
IPv4Multicast
IPv6Unicast
IPv6Multicast
Arbitrary

Brief Description

An Ethernet II frame

An ARP packet frame

An IPv4 packet frame

An IPv6 packet frame

An IPv4 unicast packet frame

An IPv4 multicast packet frame

An IPv6 unicast packet frame

An IPv6 multicast packet frame

Any type of packet frames 


\subsection{Metadata Types}

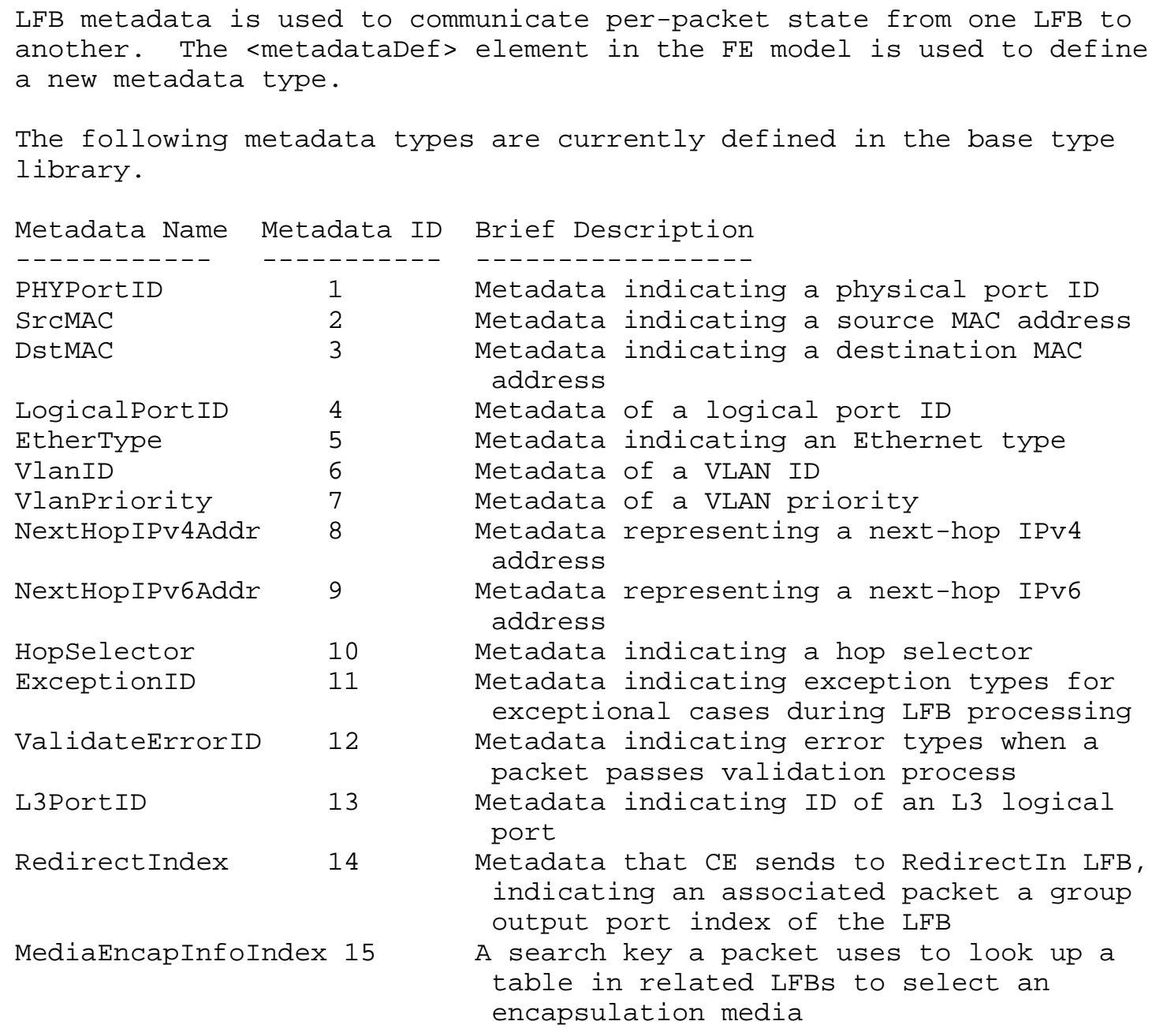




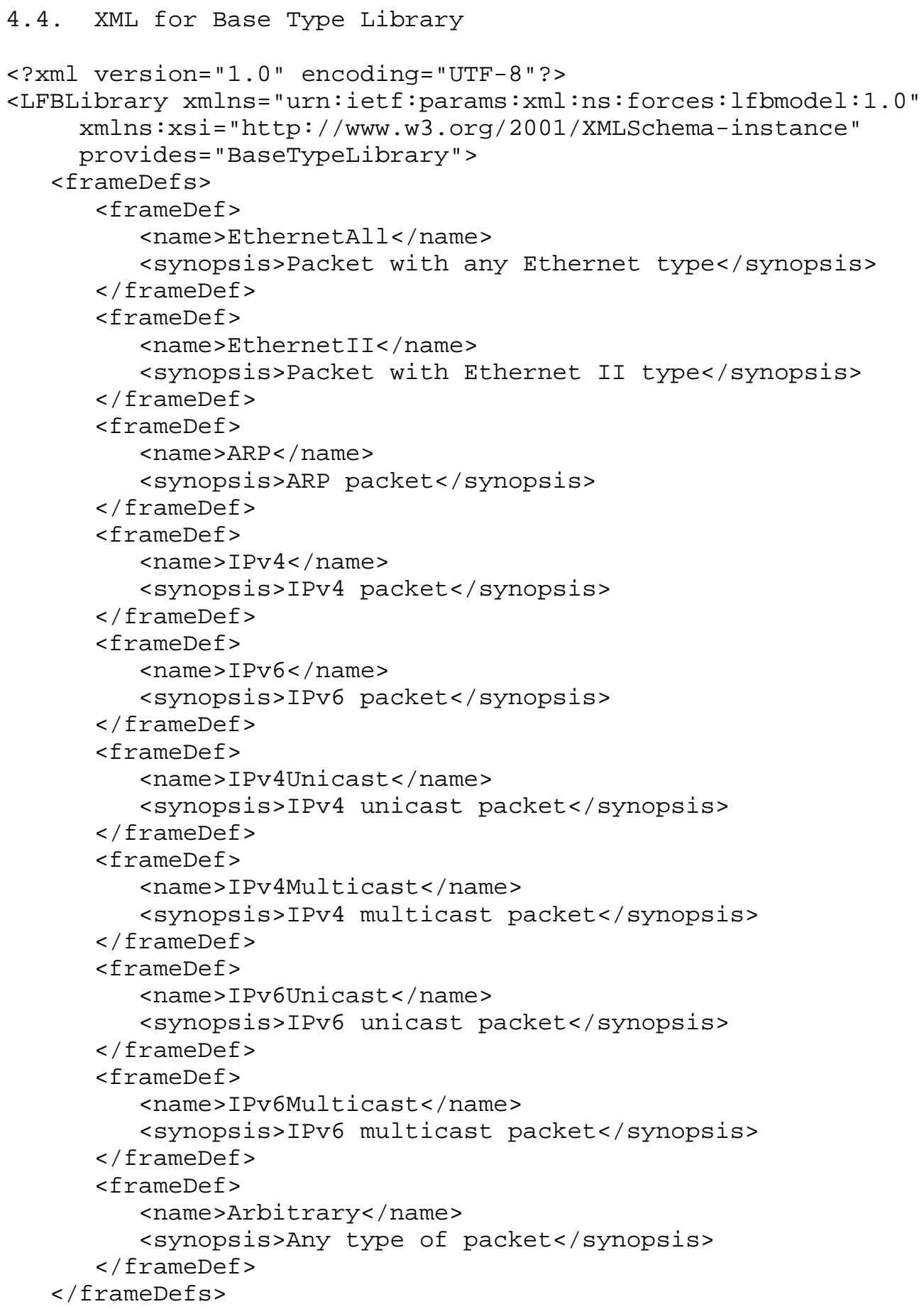




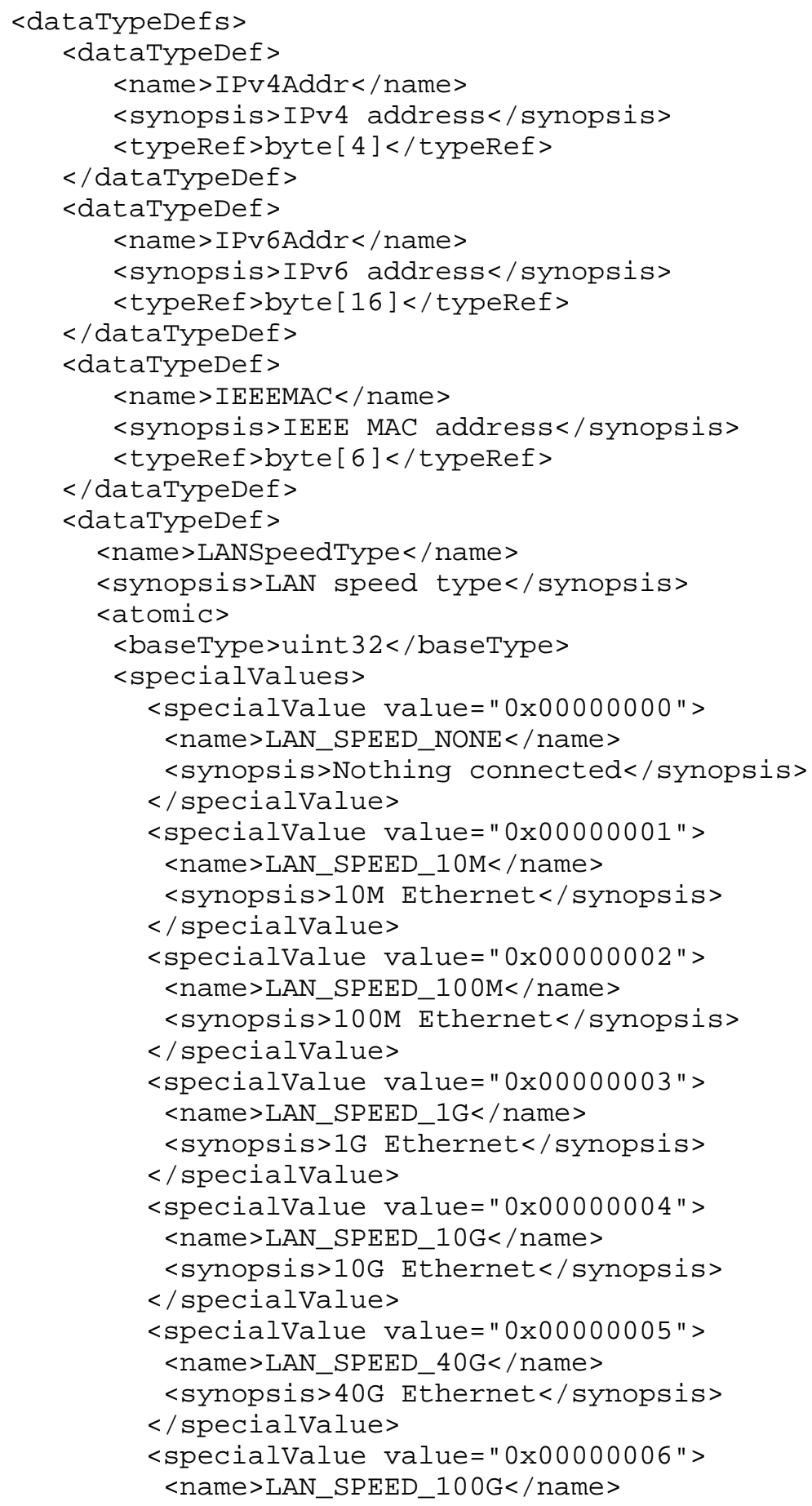




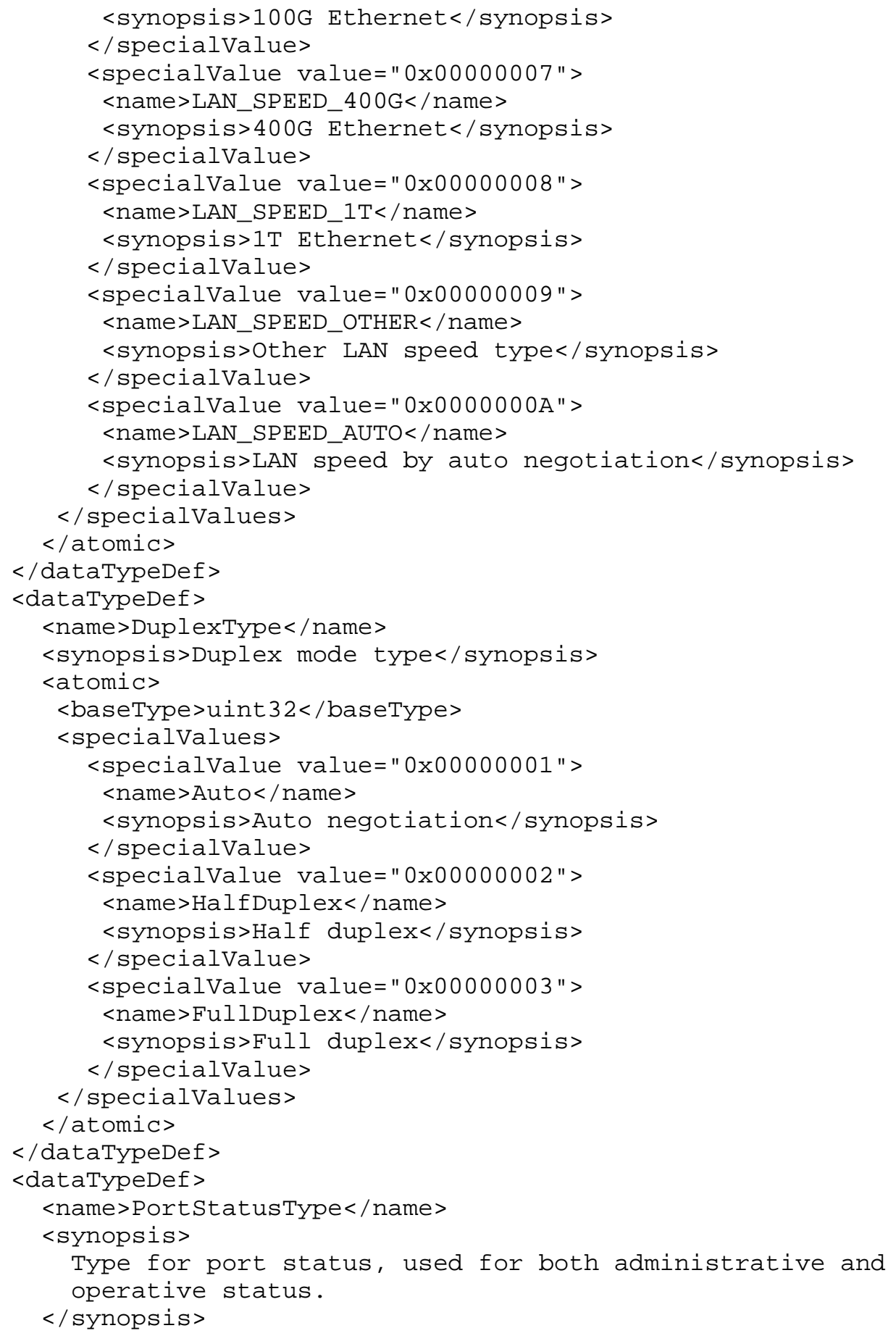




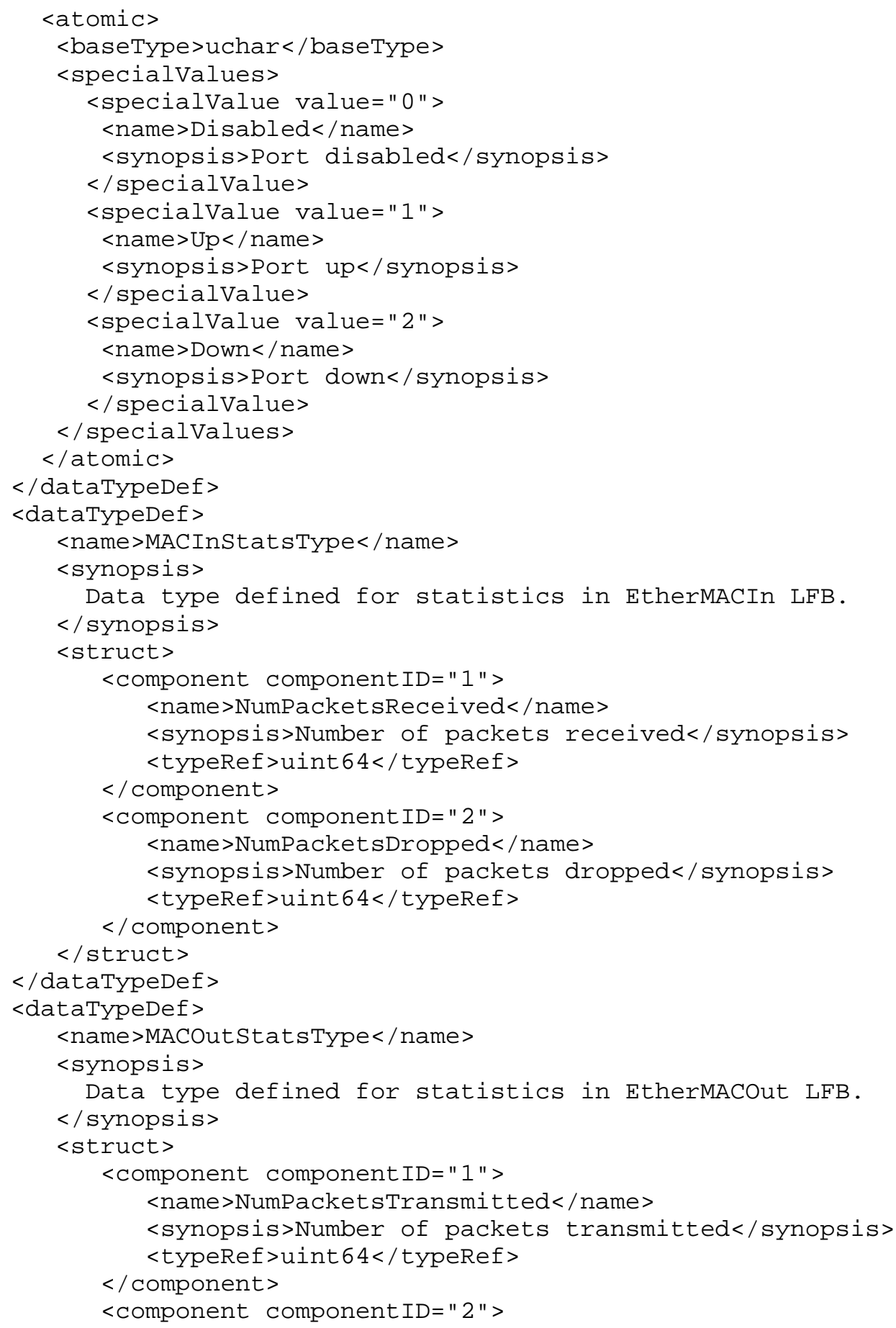




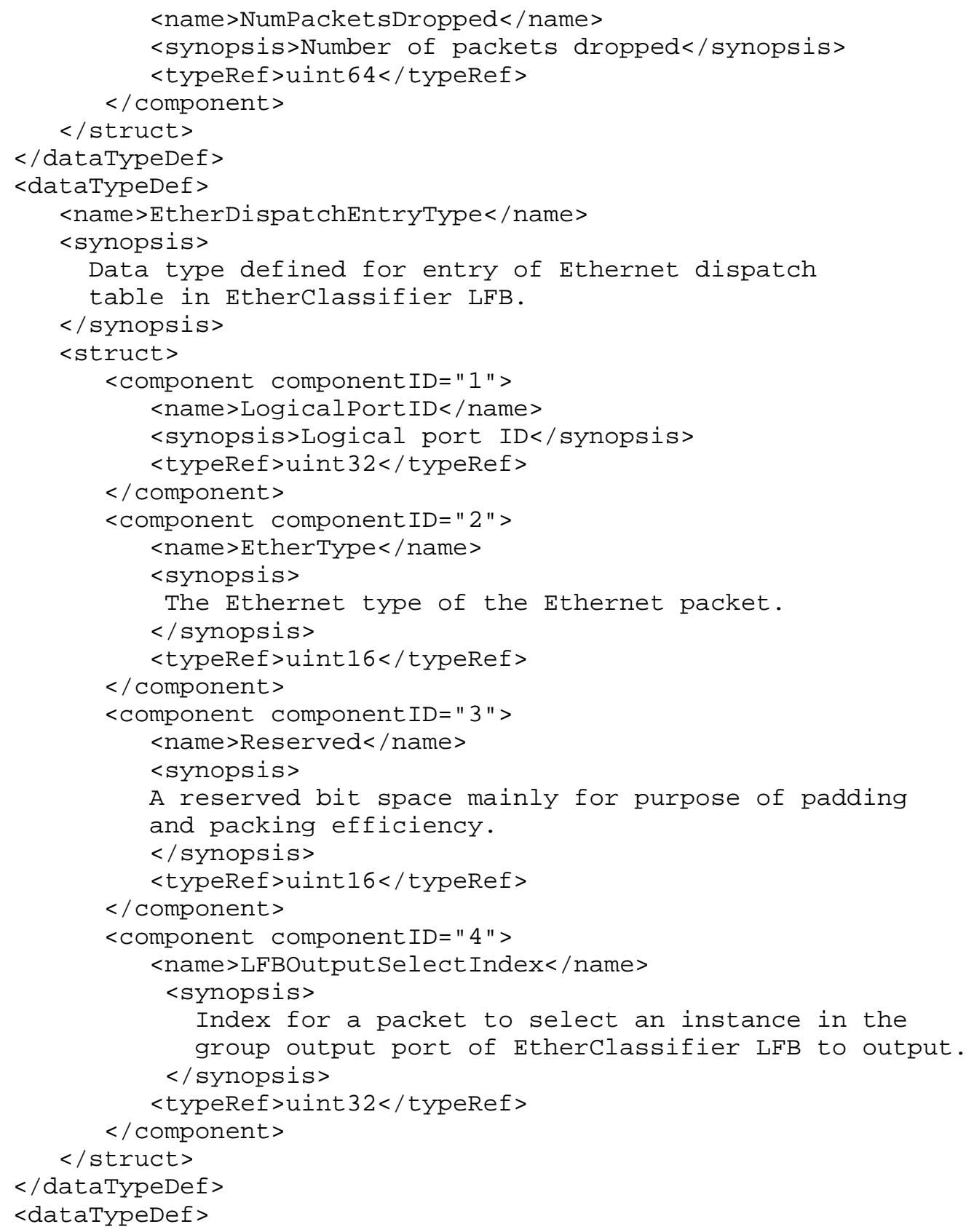




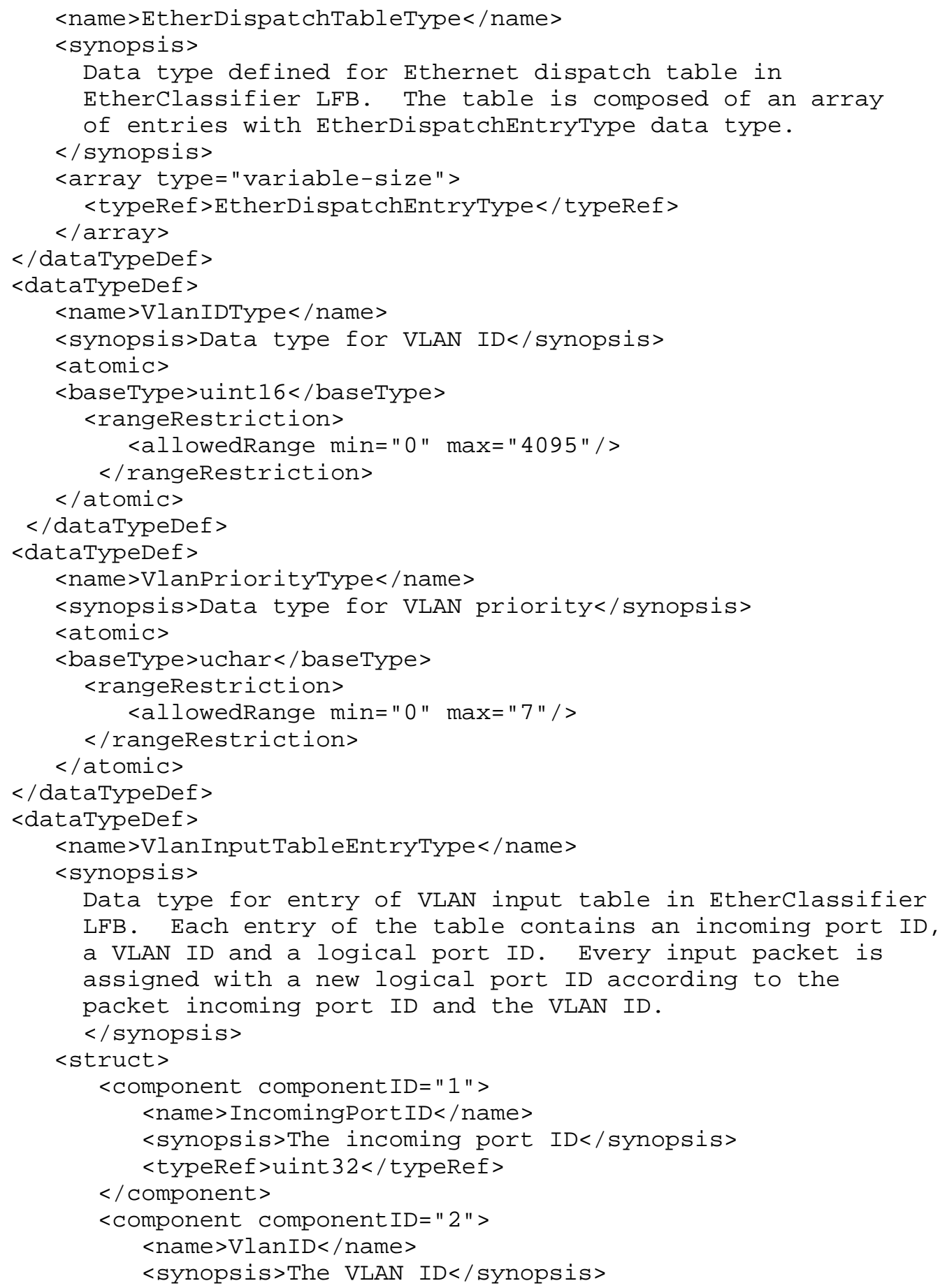




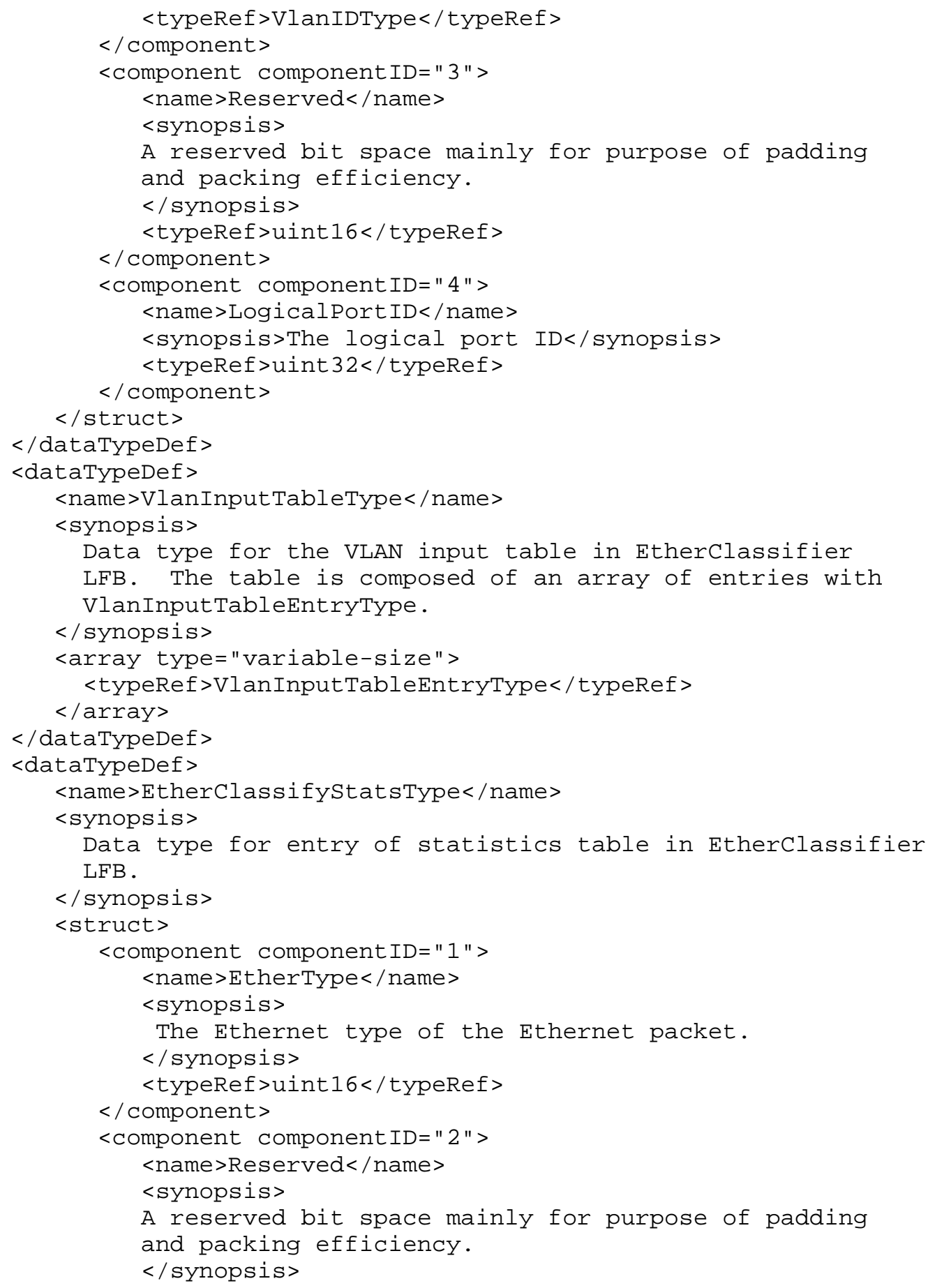




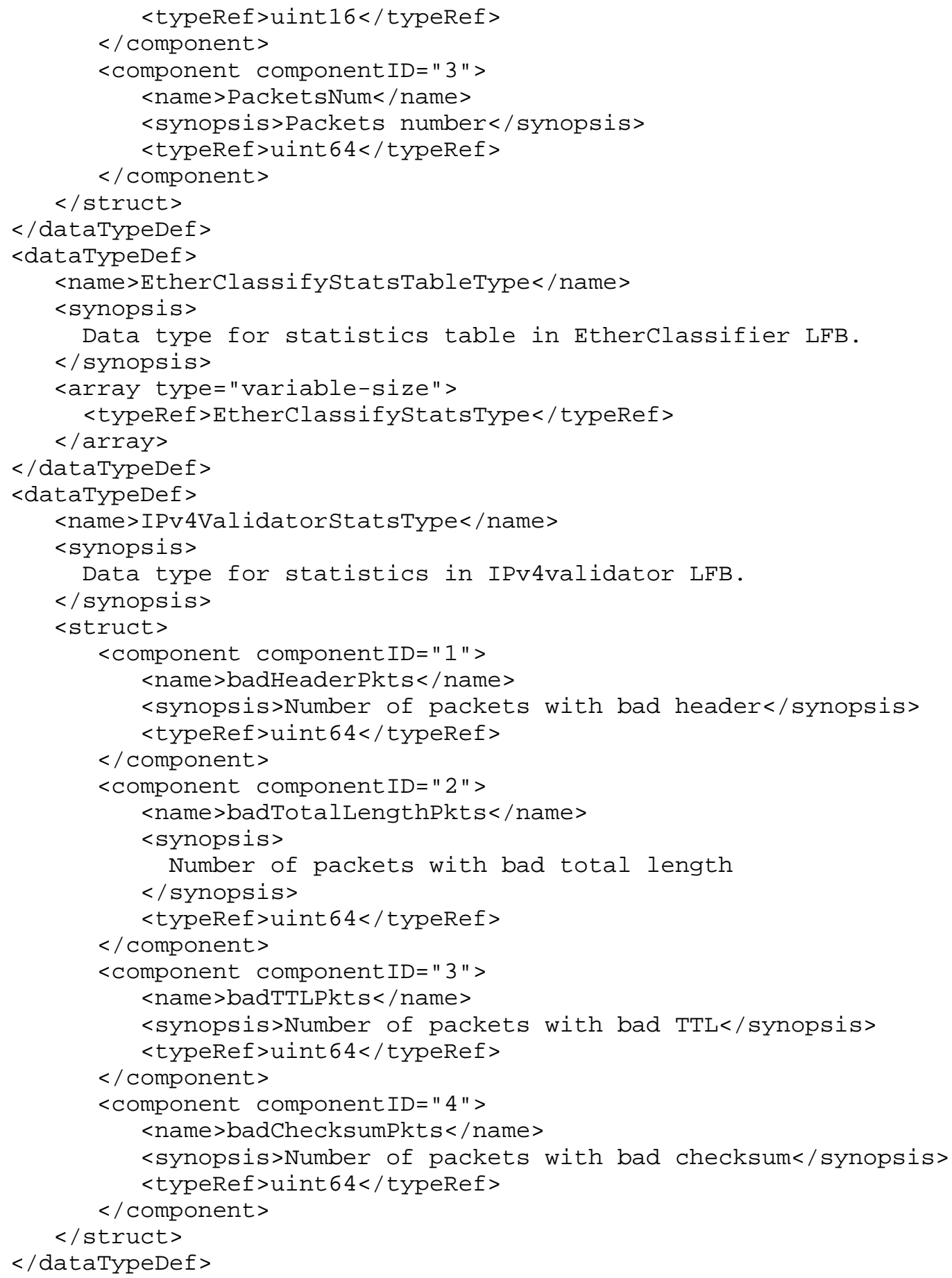




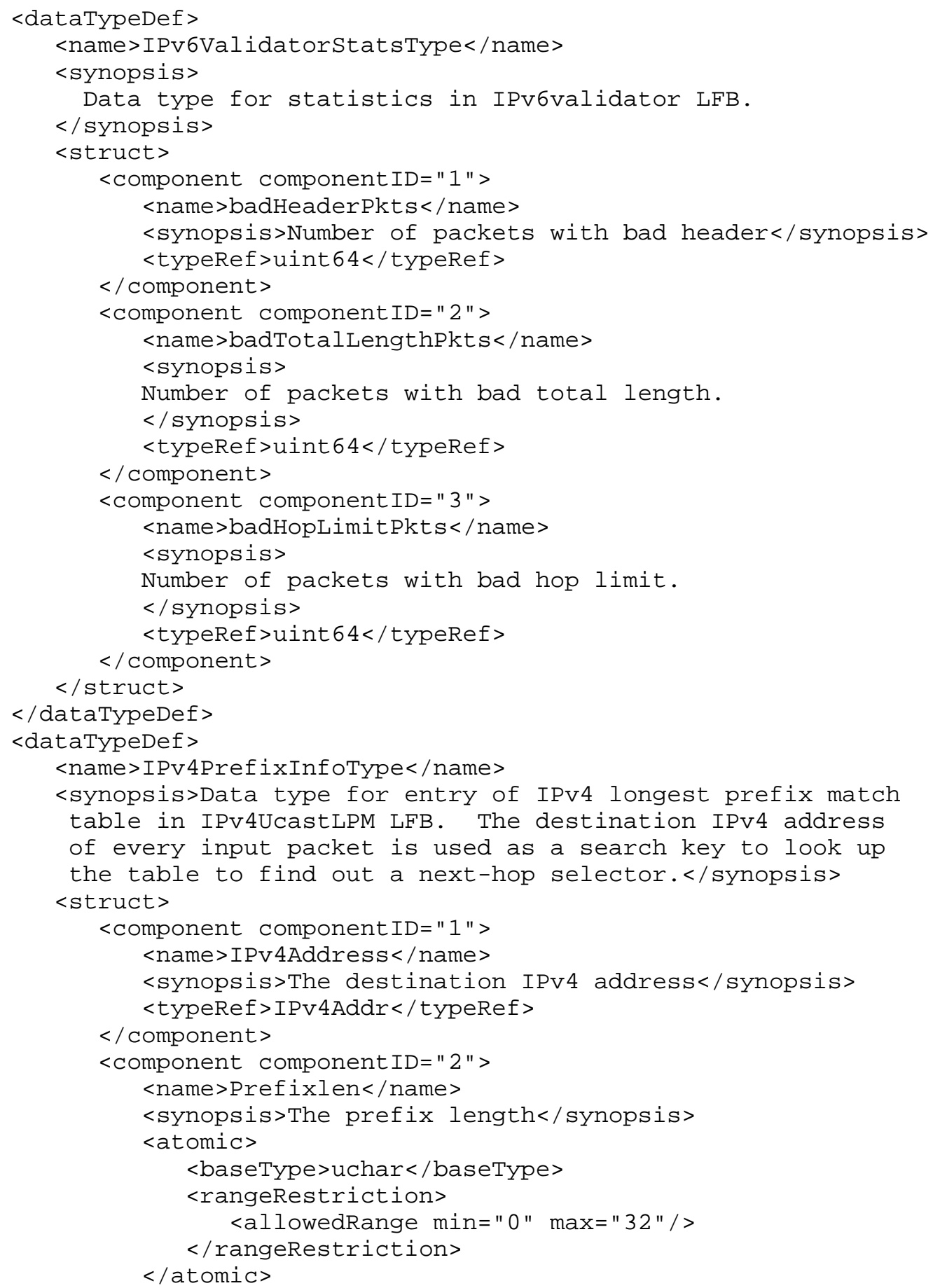

Wang, et al. 


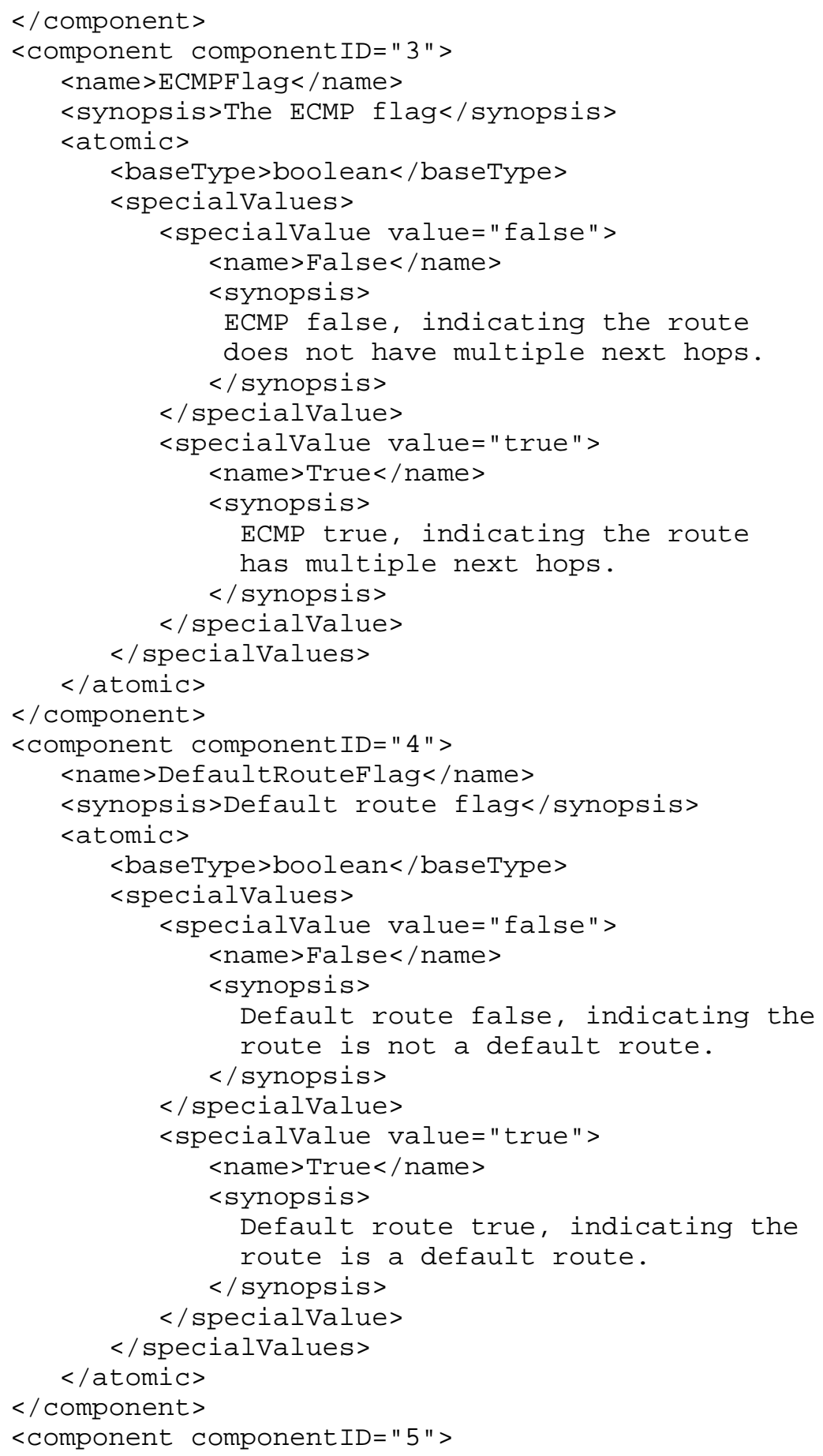

Wang, et al. 


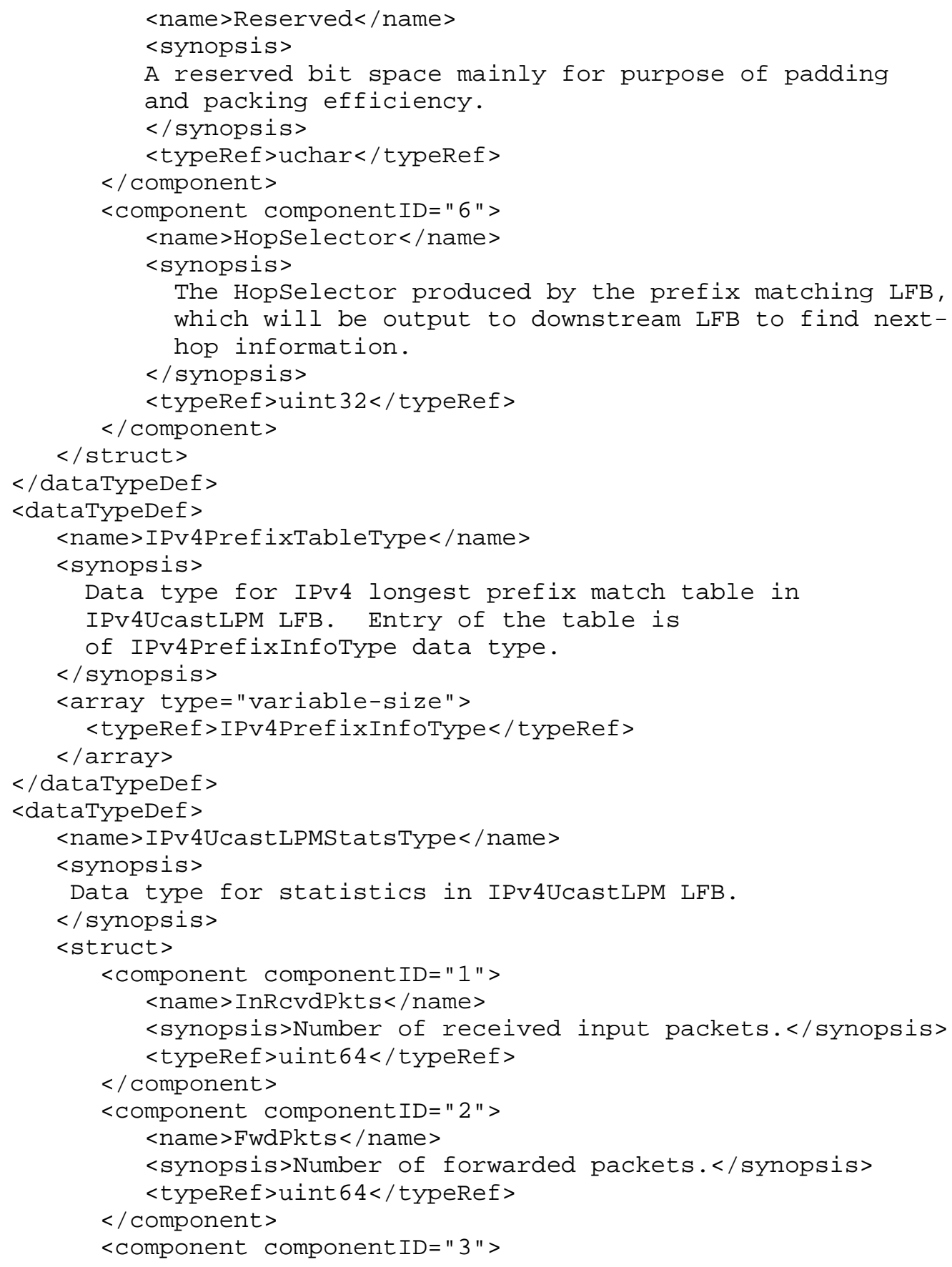




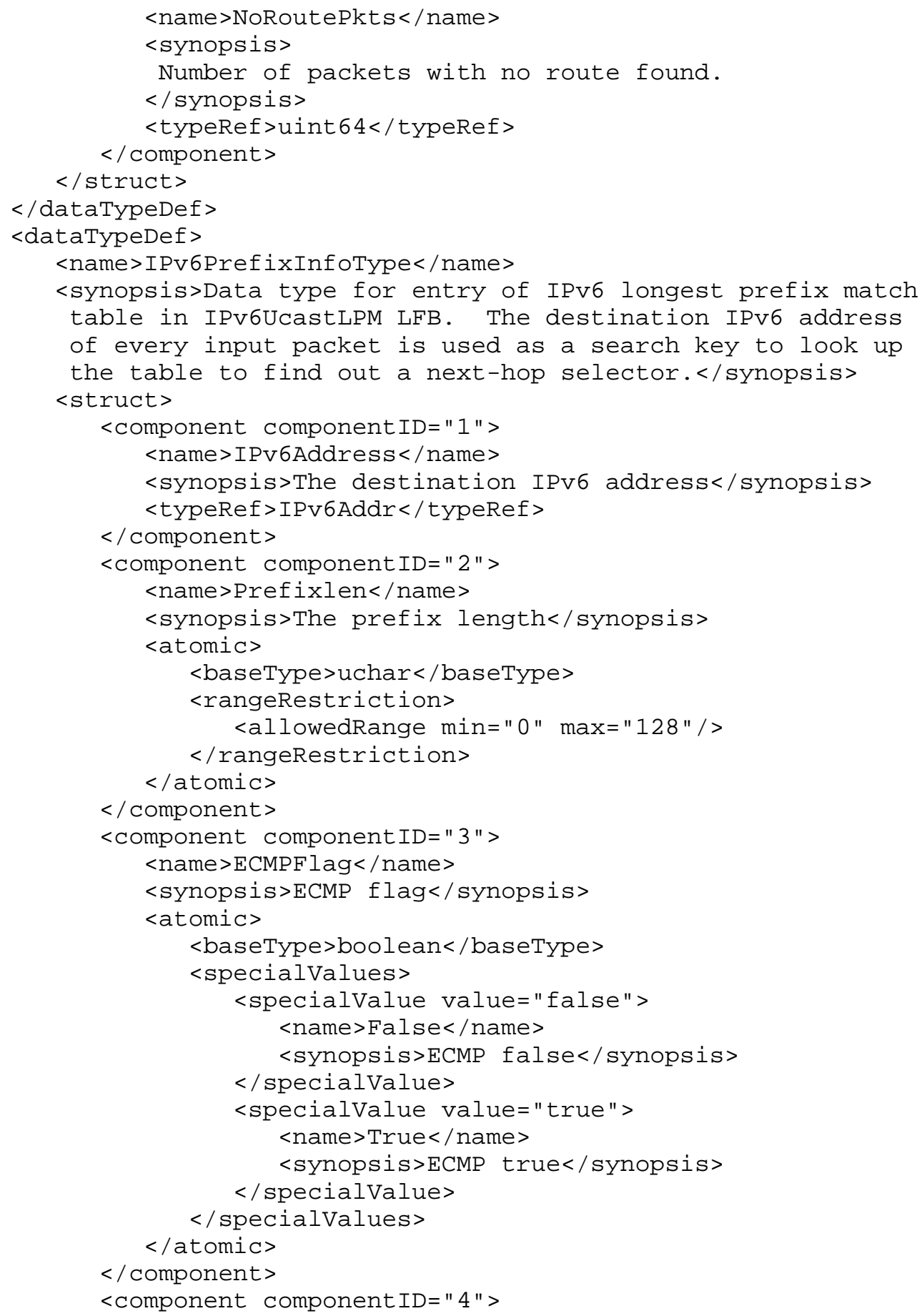

Wang, et al. 


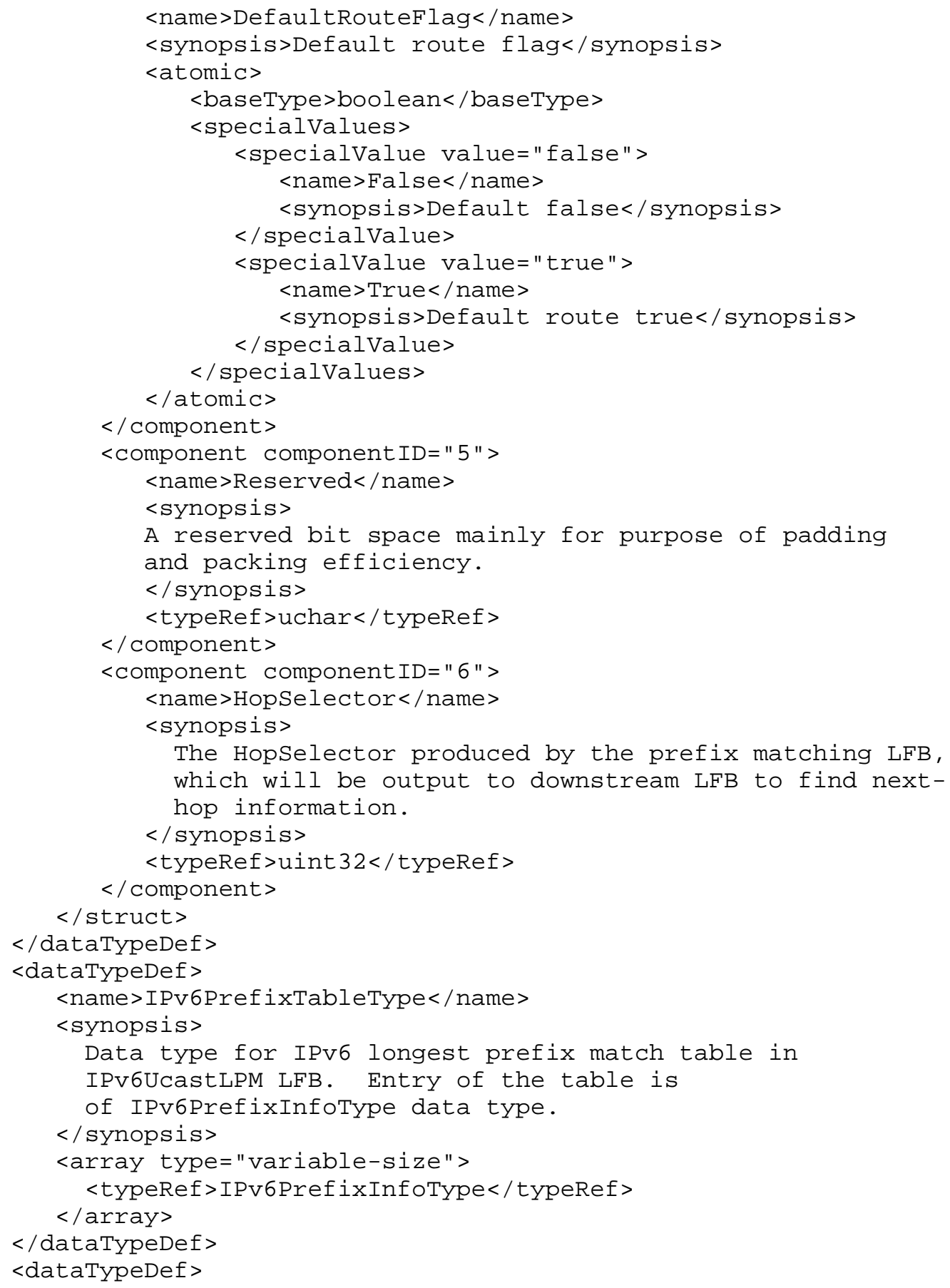




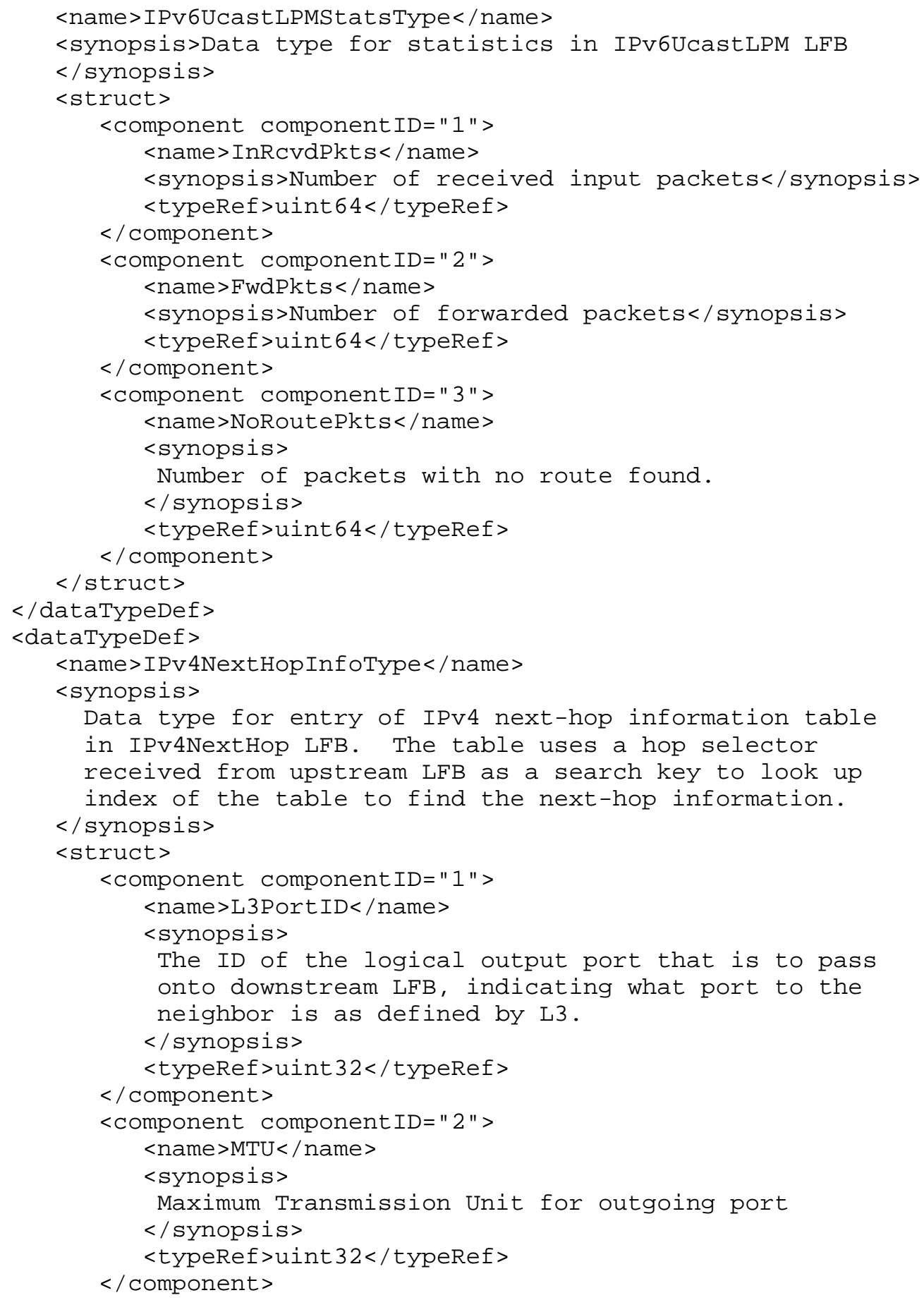

Wang, et al. 


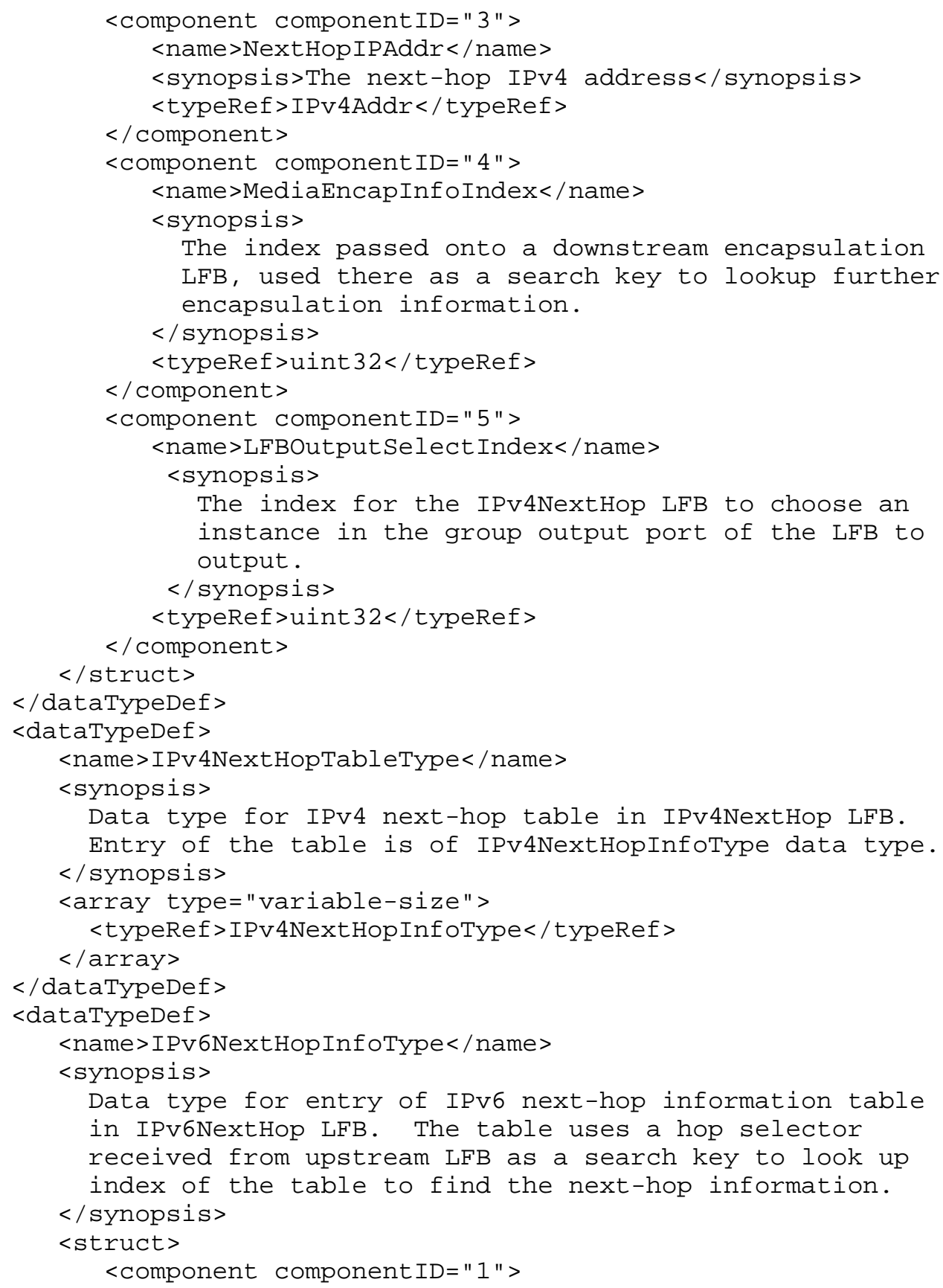




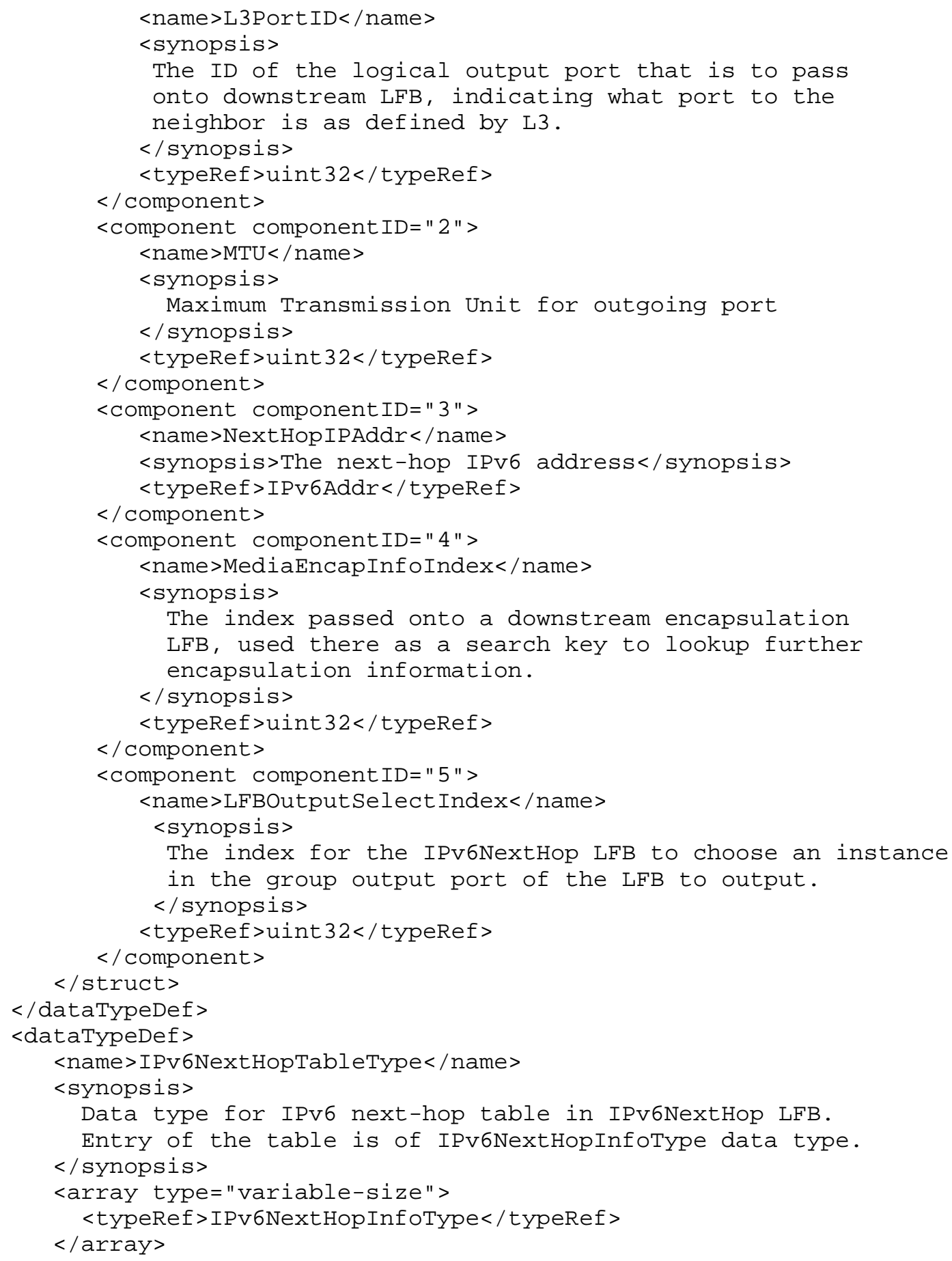




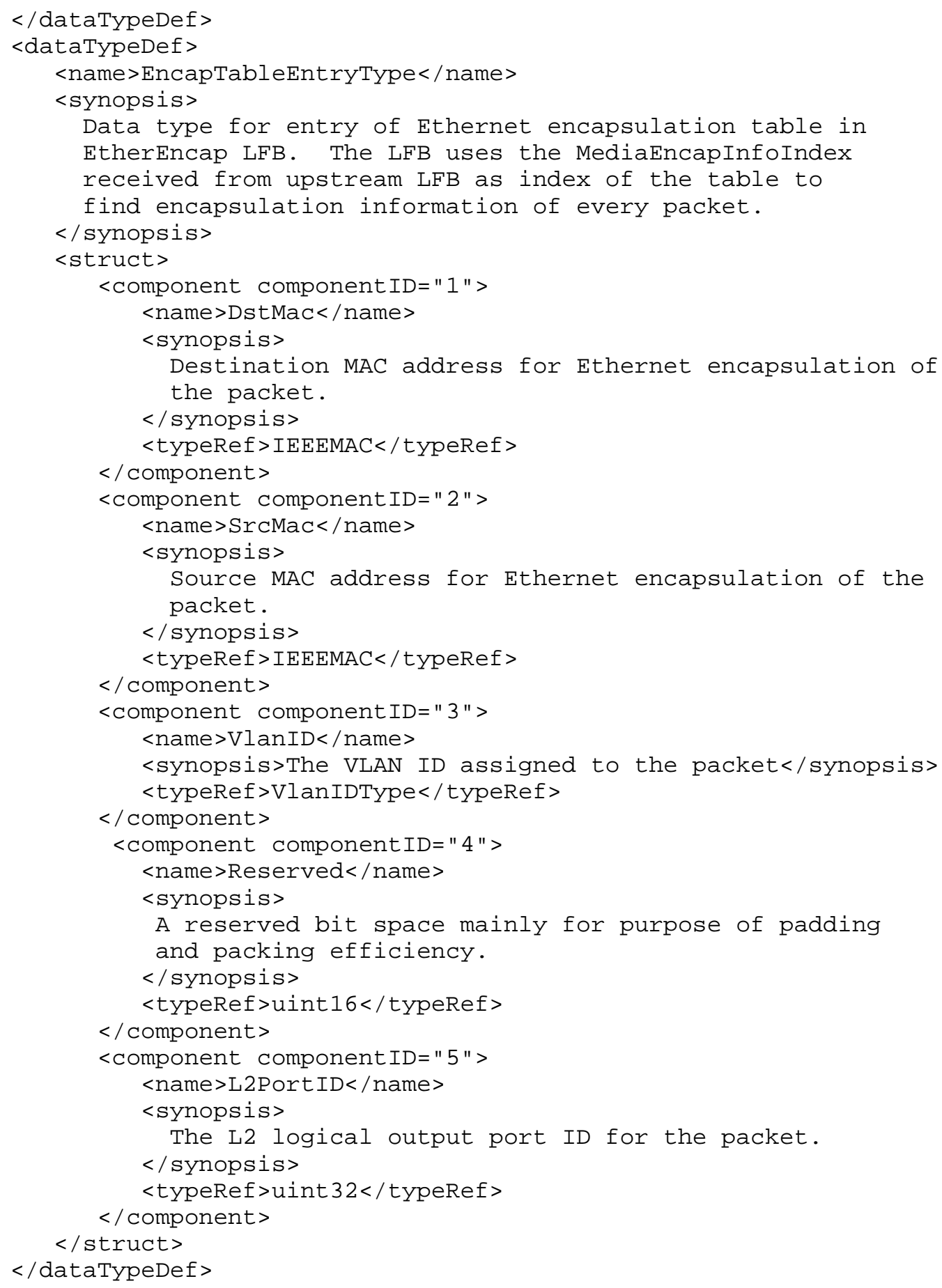

Wang, et al. 


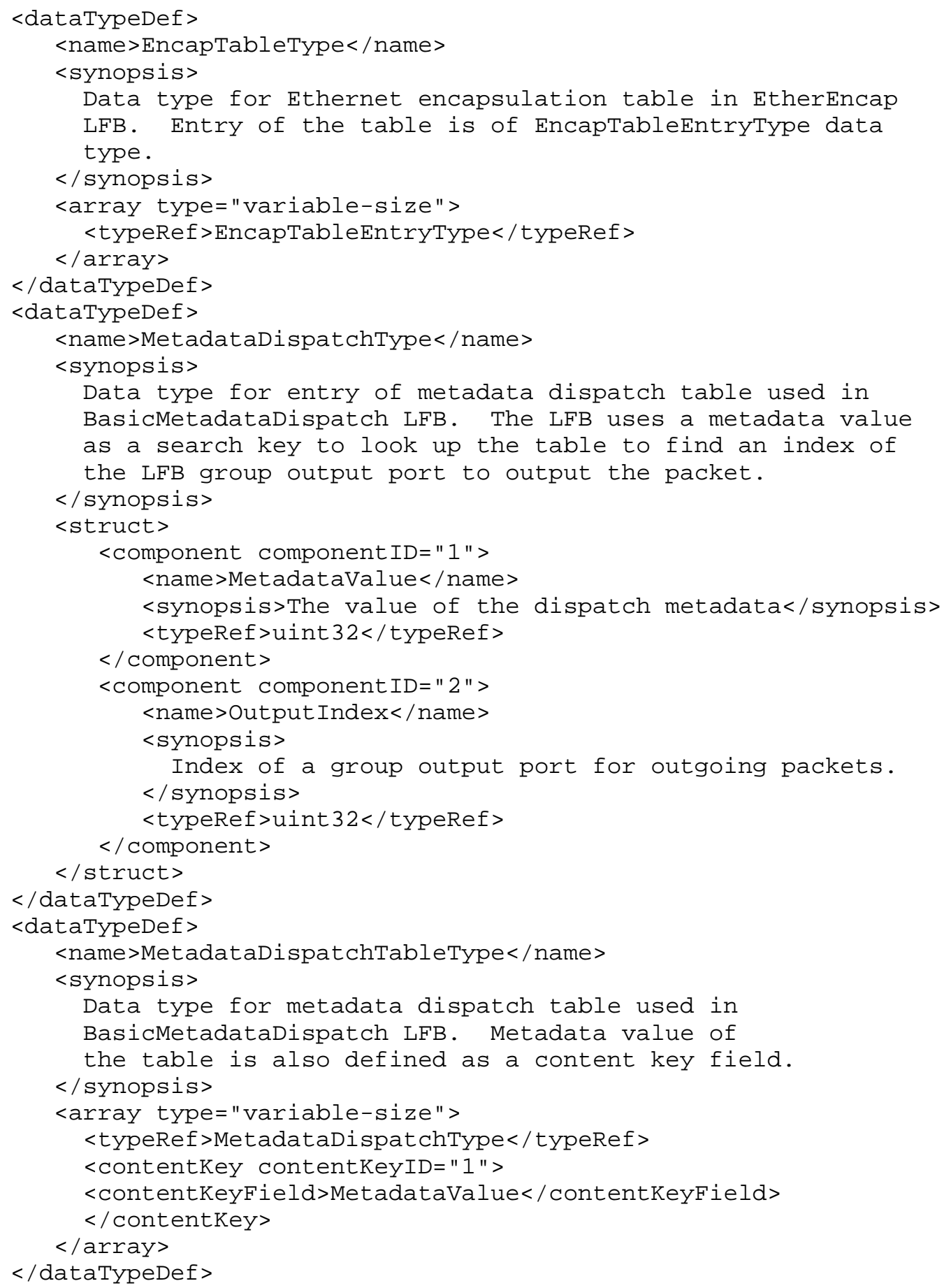

Wang, et al. 


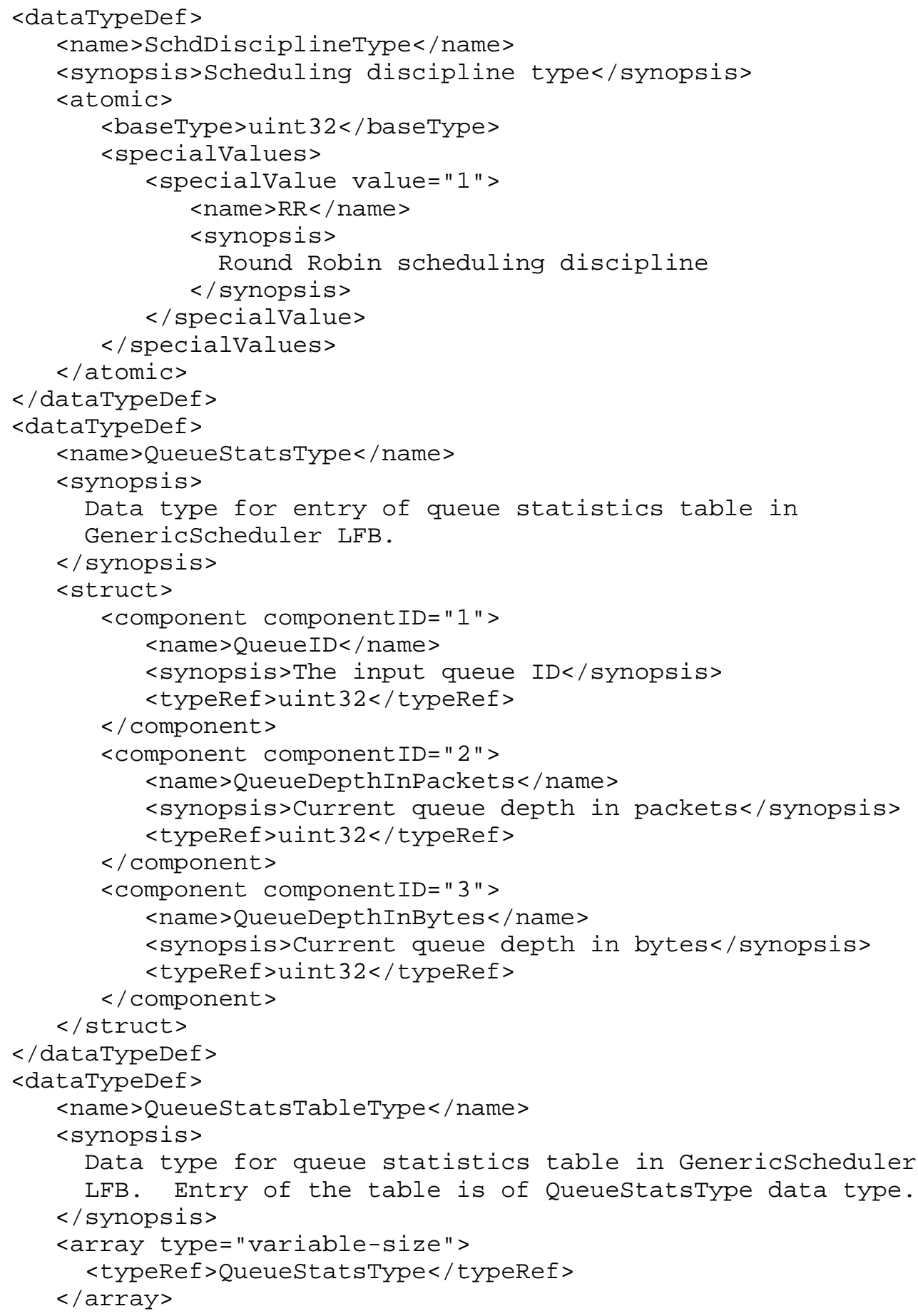




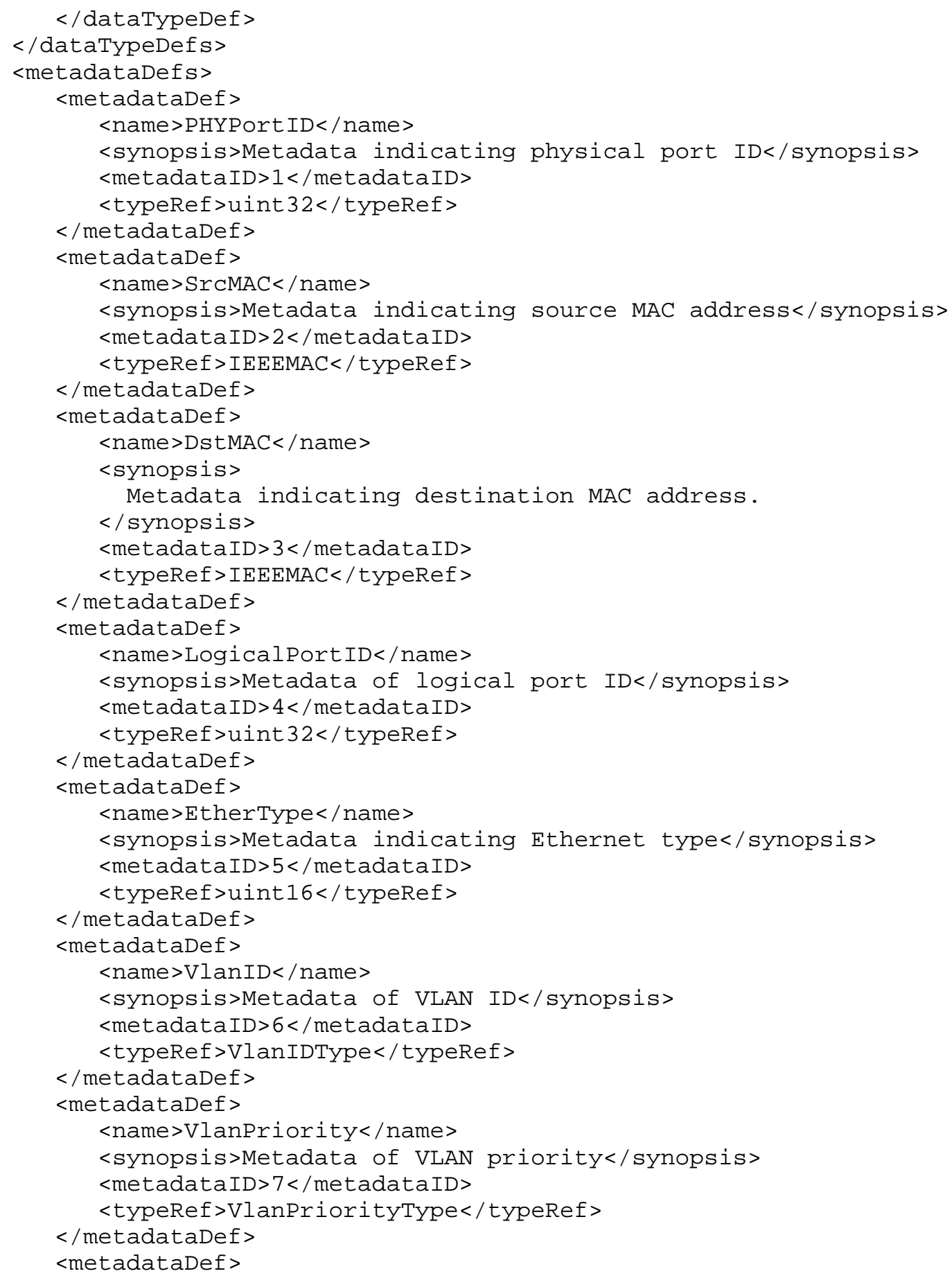




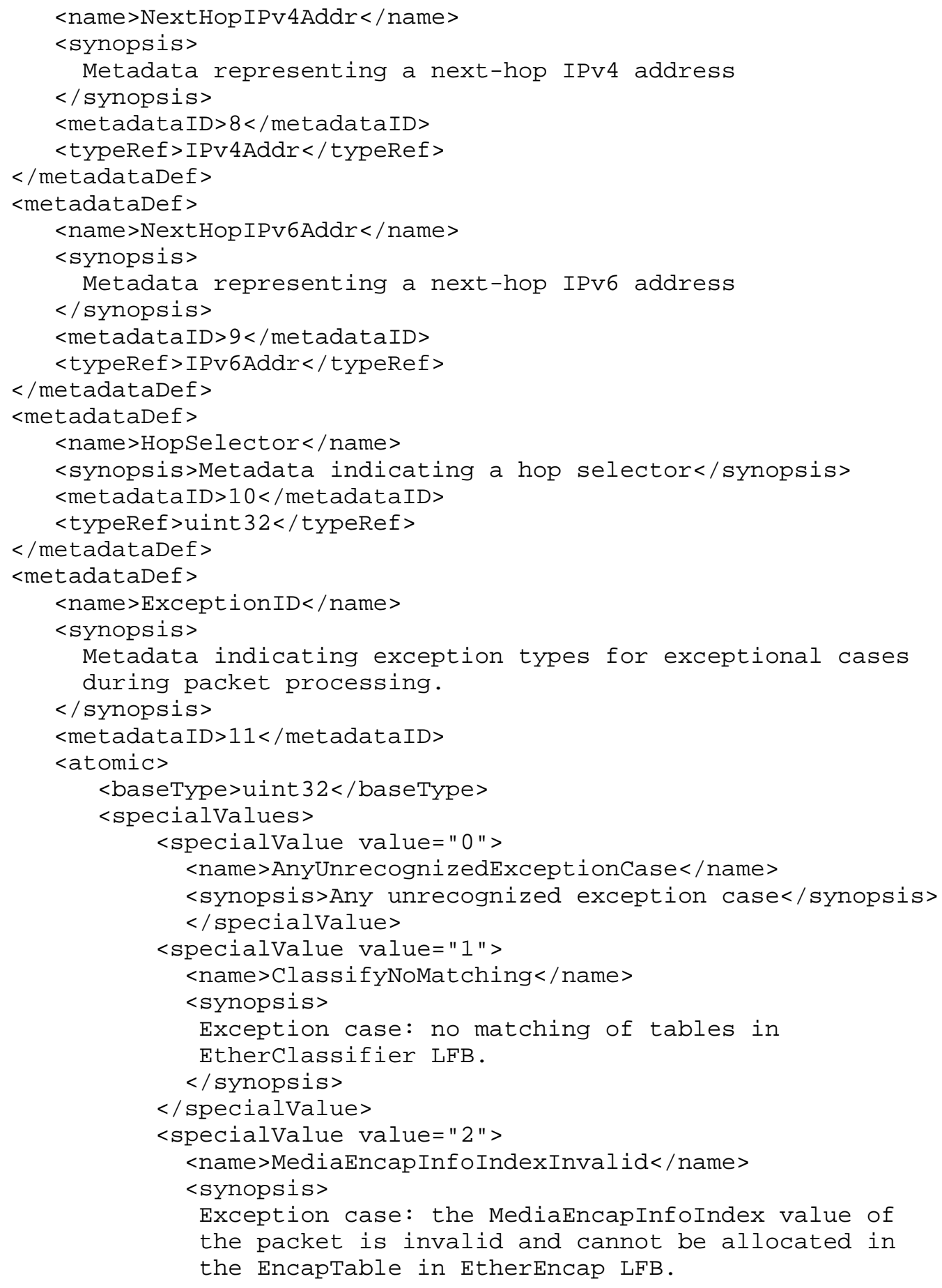




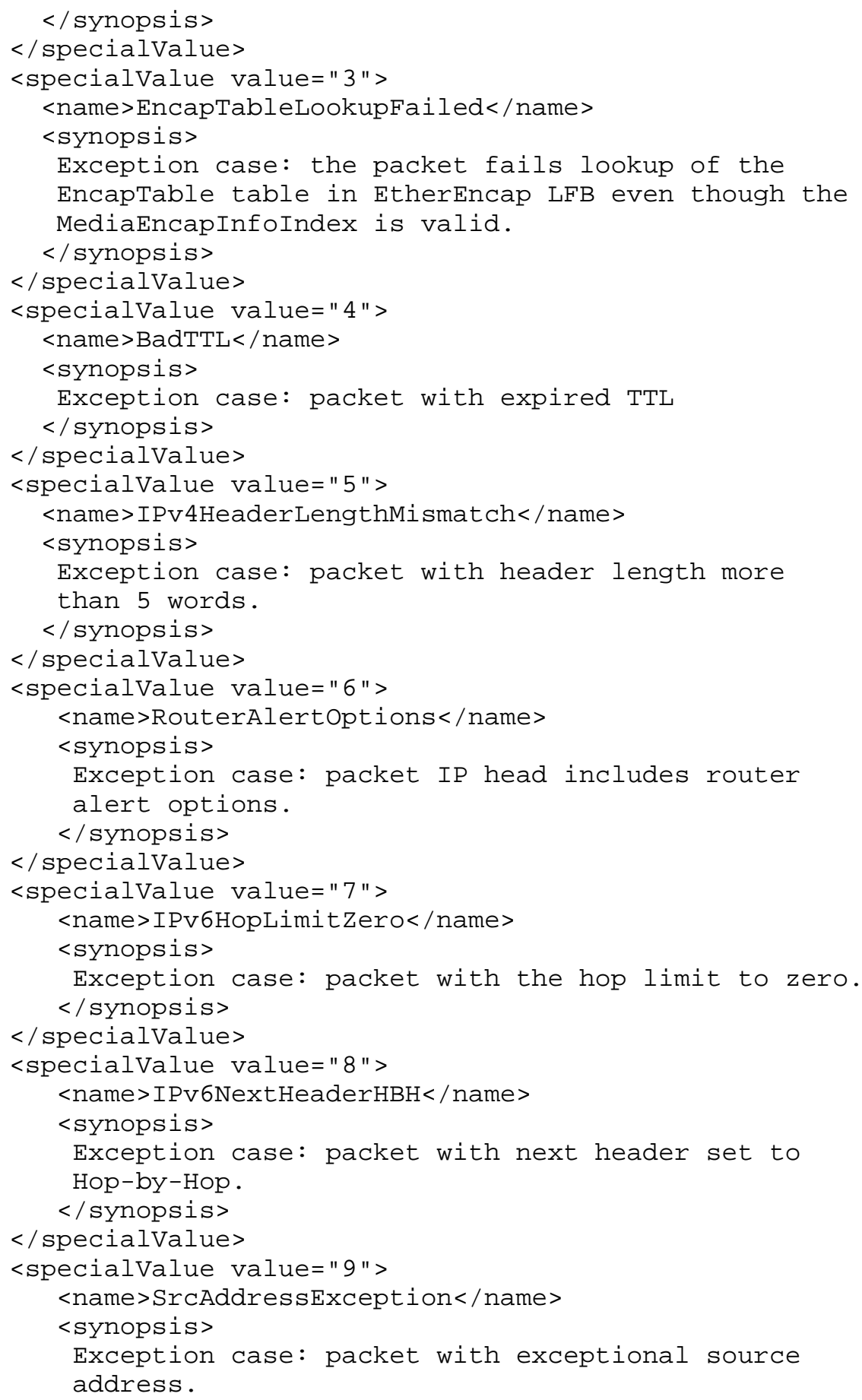




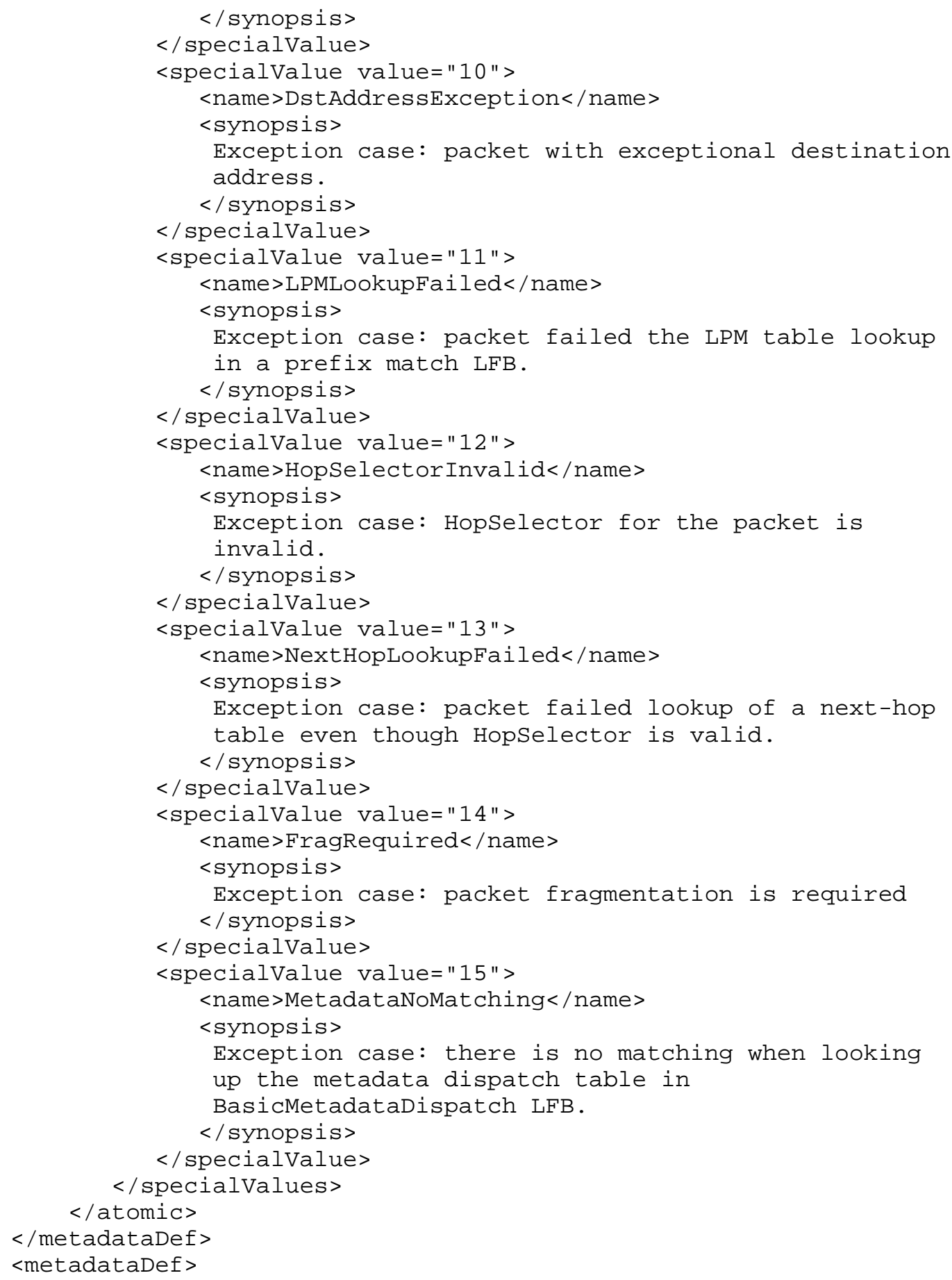




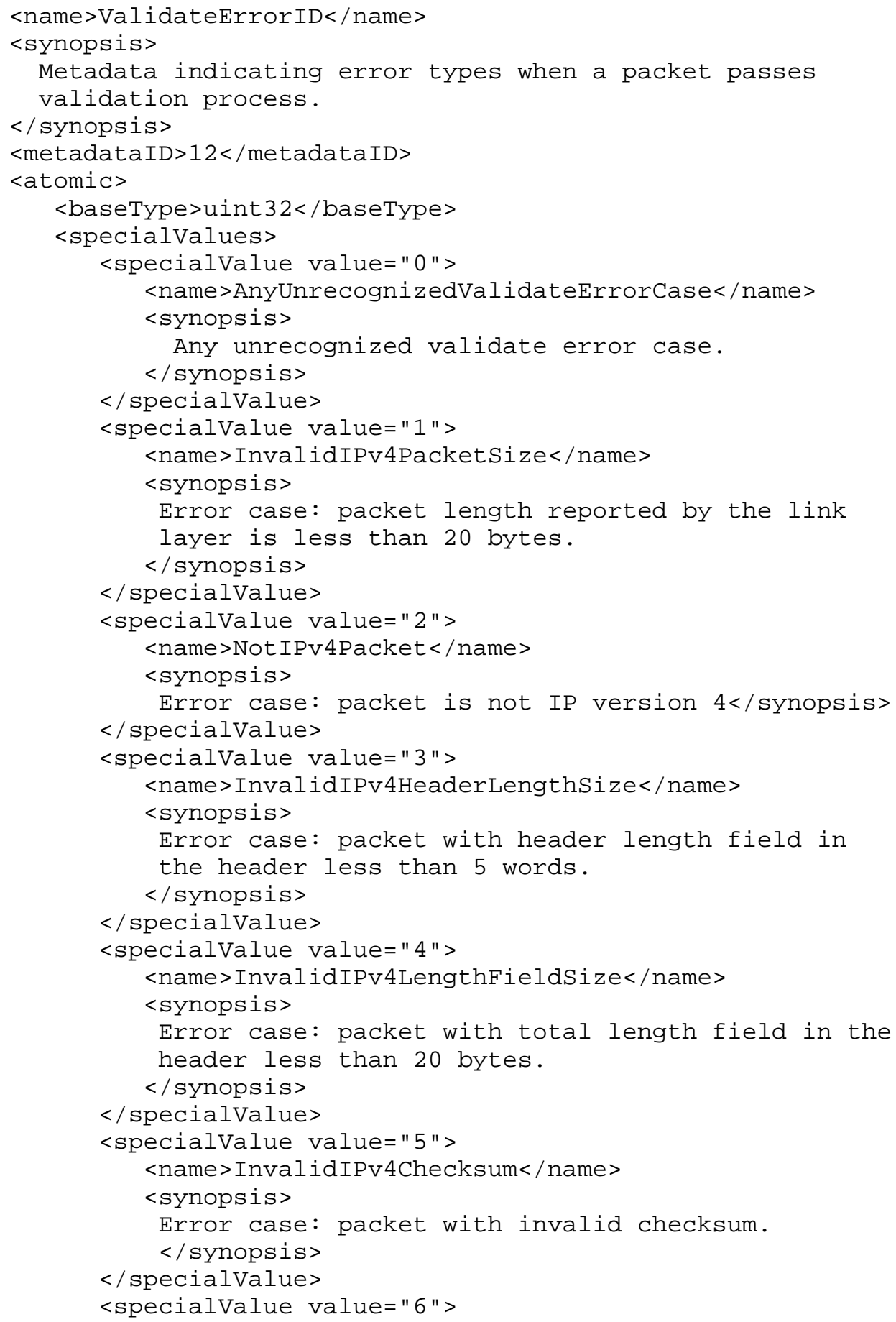

Wang, et al. 


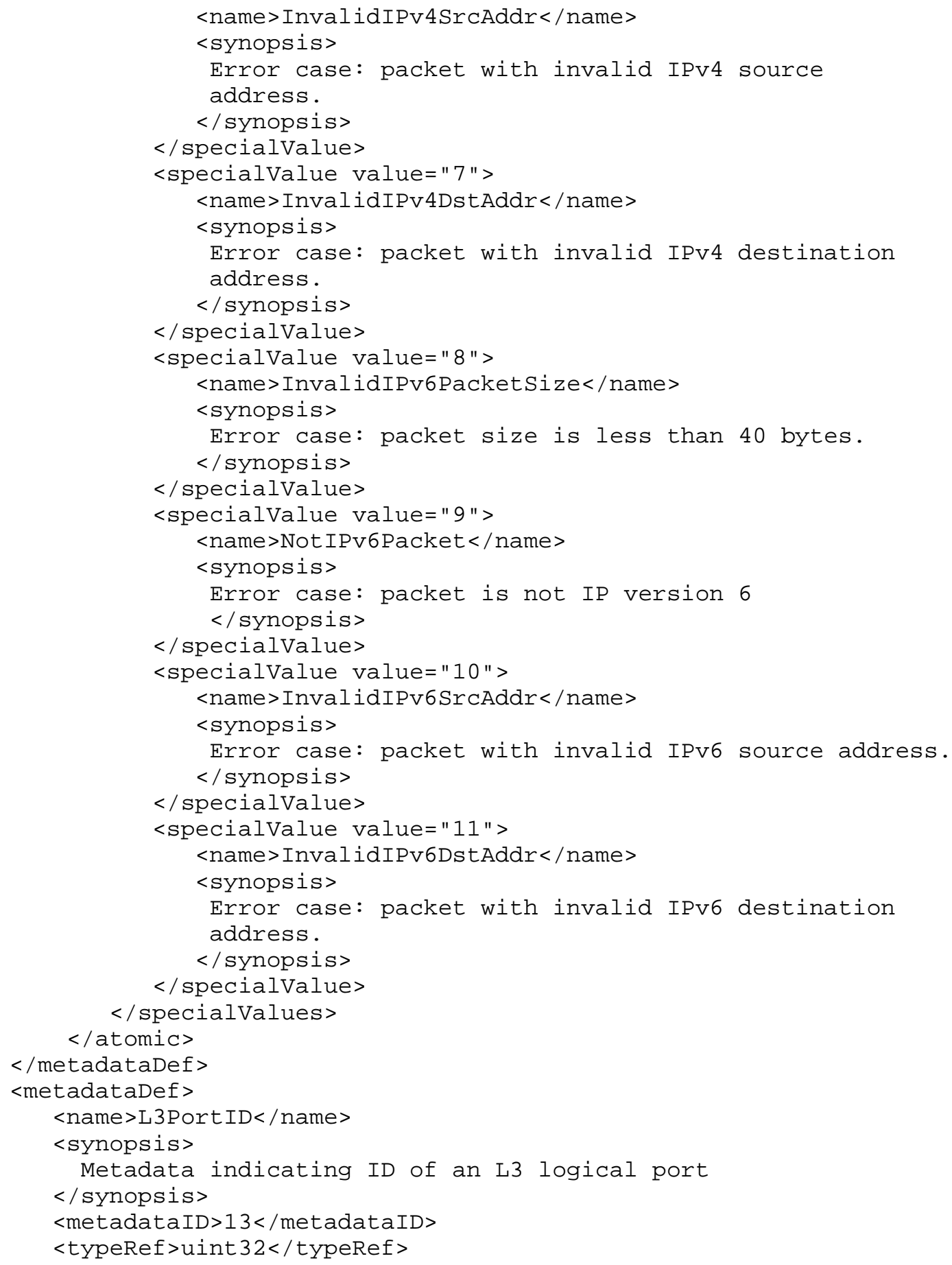




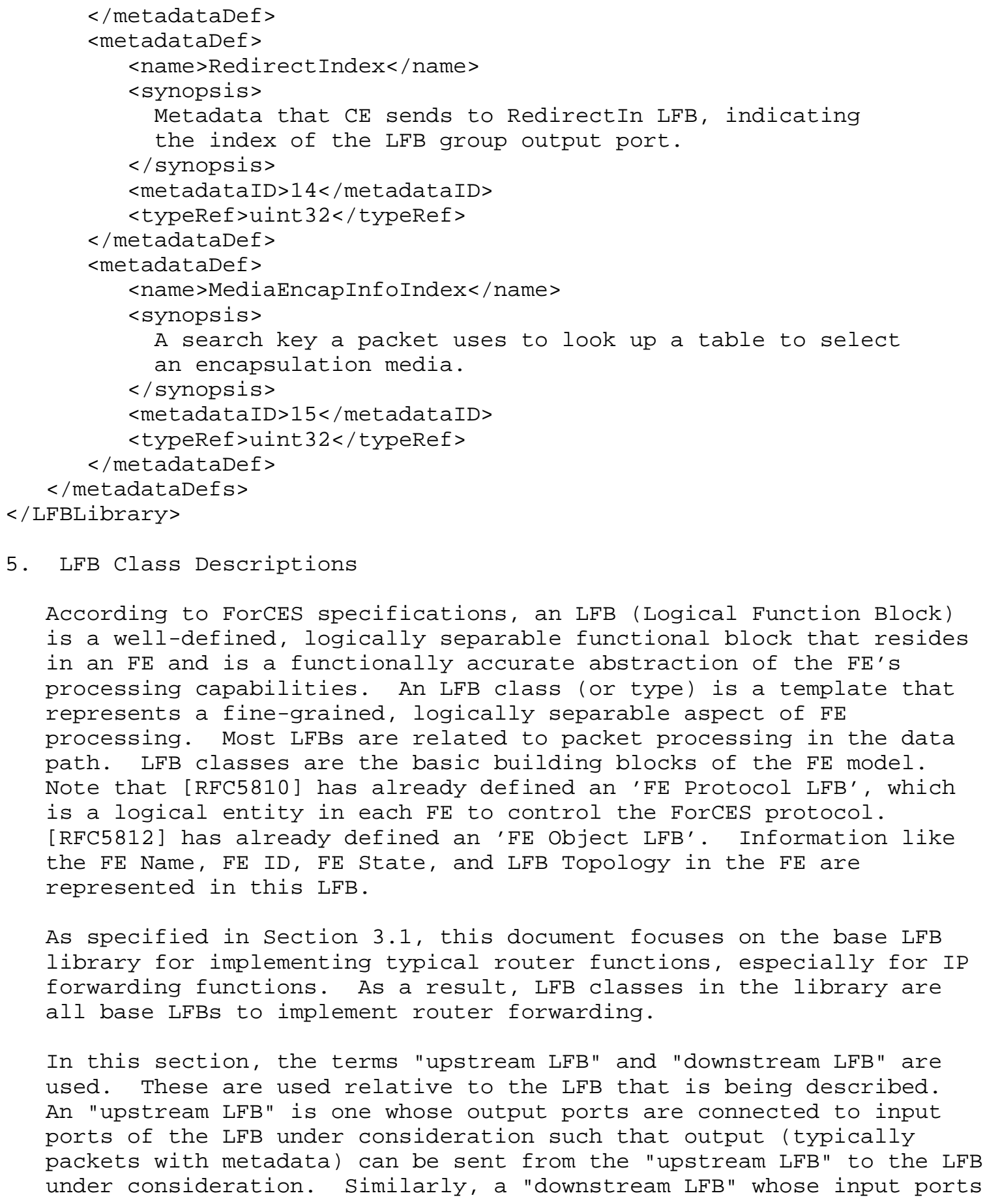


are connected to output ports of the LFB under consideration such that the LFB under consideration can send information to the "downstream LFB". Note that in some rare topologies, an LFB may be both upstream and downstream relative to another LFB.

Also note that, as a default provision of [RFC5812], in the FE model, all metadata produced by upstream LFBs will pass through all downstream LFBs by default without being specified by input port or output port. Only those metadata that will be used (consumed) by an LFB will be explicitly marked in the input of the LFB as expected metadata. For instance, in downstream LFBs of a physical-layer LFB, even if there is no specific metadata expected, metadata like PHYPortID produced by the physical-layer LFB will always pass through all downstream LFBs regardless of whether or not the metadata has been expected by the LFBs.

\subsection{Ethernet-Processing LFBs}

As the most popular physical- and data-link-layer protocol, Ethernet is widely deployed. It becomes a basic requirement for a router to be able to process various Ethernet data packets.

Note that different versions of Ethernet formats exist, like Ethernet V2, 802.3 RAW, IEEE 802.3/802.2, and IEEE 802.3/802.2 SNAP. Varieties of LAN techniques based on Ethernet also exist, like various VLANs, MACinMAC, etc. Ethernet-processing LFBs defined here are intended to be able to cope with all these variations of Ethernet technology.

There are also various types of Ethernet physical interface media. Among them, copper and fiber media may be the most popular ones. As a base LFB definition and a starting point, this document only defines an Ethernet physical LFB with copper media. For other media interfaces, specific LFBs may be defined in future versions of the library.

\subsubsection{EtherPHYCop}

EtherPHYCOP LFB abstracts an Ethernet interface physical layer with media limited to copper.

\subsubsection{Data Handling}

This LFB is the interface to the Ethernet physical media. The LFB handles Ethernet frames coming in from or going out of the FE. Ethernet frames sent and received cover all packets encapsulated with different versions of Ethernet protocols, like Ethernet V2, 802.3 RAW, IEEE 802.3/802.2, and IEEE 802.3/802.2 SNAP, including packets 
encapsulated with varieties of LAN techniques based on Ethernet, like various VLANs, MACinMAC, etc. Therefore, in the XML, an EthernetAll frame type has been introduced.

Ethernet frames are received from the physical media port and passed downstream to LFBs, such as EtherMACIn LFBs, via a singleton output known as "EtherPHYOut". A PHYPortID metadata, which indicates the physical port from which the frame came in from the external world, is passed along with the frame.

Ethernet packets are received by this LFB from upstream LFBs, such as EtherMacOut LFBs, via the singleton input known as "EtherPHYIn" before being sent out to the external world.

\subsubsection{Components}

The Adminstatus component is defined for the CE to administratively manage the status of the LFB. The CE may administratively start up or shut down the LFB by changing the value of Adminstatus. The default value is set to 'Down'.

An OperStatus component captures the physical port operational status. A PHYPortstatuschanged event is defined so the LFB can report to the $\mathrm{CE}$ whenever there is an operational status change of the physical port.

The PHYPortID component is a unique identification for a physical port. It is defined as 'read-only' by the CE. Its value is enumerated by FE. The component will be used to produce a PHYPortID metadata at the LFB output and to associate it to every Ethernet packet this LFB receives. The metadata will be handed to downstream LFBs for them to use the PHYPortID.

A group of components are defined for link speed management. The Adminlinkspeed is for the CE to configure link speed for the port, and the operLinkspeed is for the CE to query the actual link speed in operation. The default value for the AdminLinkspeed is set to autonegotiation mode.

A group of components are defined for duplex mode management. The AdminDuplexMode is for the CE to configure proper duplex mode for the port, and the OperDuplexMode is for $\mathrm{CE}$ to query the actual duplex mode in operation. The default value for the AdminDuplexMode is set to auto-negotiation mode.

A Carrierstatus component captures the status of the carrier and specifies whether the port link is operationally up. The default value for the Carrierstatus is 'false'. 


\subsubsection{Capabilities}

The capability information for this LFB includes the link speeds that are supported by the FE (SupportedLinkSpeed) as well as the supported duplex modes (SupportedDuplexMode).

\subsubsection{Events}

Several events are generated. There is an event for changes in the status of the physical port (Phyportstatuschanged). Such an event will notify that the physical port status has been changed, and the report will include the new status of the physical port.

Another event captures changes in the operational link speed (LinkSpeedChanged). Such an event will notify the CE that the operational speed has been changed, and the report will include the new negotiated operational speed.

A final event captures changes in the duplex mode (DuplexModeChanged). Such an event will notify the CE that the duplex mode has been changed and the report will include the new negotiated duplex mode.

\subsubsection{EtherMACIn}

EtherMACIn LFB abstracts an Ethernet port at the MAC data link layer. This LFB describes Ethernet processing functions like checking MAC address locality, deciding if the Ethernet packets should be bridged, providing Ethernet-layer flow control, etc.

\subsubsection{Data Handling}

The LFB is expected to receive all types of Ethernet packets (via a singleton input known as "EtherPktsIn"), which are usually output from some Ethernet physical-layer LFB, like an EtherPHYCop LFB, along with a metadata indicating the physical port ID of the port on which the packet arrived.

The LFB is defined with two separate singleton outputs. All output packets are emitted in the original Ethernet format received at the physical port, unchanged, and cover all Ethernet types.

The first singleton output is known as "Normalpathout". It usually outputs Ethernet packets to some LFB, like an EtherClassifier LFB, for further L3 forwarding process along with a PHYPortID metadata indicating the physical port from which the packet came. 
The second singleton output is known as "L2BridgingPathout" . Although the LFB library this document defines is basically to meet typical router functions, it will attempt to be forward compatible with future router functions. The L2BridgingPathout is defined to meet the requirement that L2 bridging functions may be optionally supported simultaneously with L3 processing and some L2 bridging LFBs that may be defined in the future. If the FE supports L2 bridging, the CE can enable or disable it by means of a "L2BridgingPathEnable" component in the $\mathrm{FE}$. If it is enabled, by also instantiating some L2 bridging LFB instances following the L2BridgingPathout, FEs are expected to fulfill L2 bridging functions. L2BridgingPathout will output packets exactly the same as in the Normalpathout output.

This LFB can be set to work in a promiscuous mode, allowing all packets to pass through the LFB without being dropped. Otherwise, a locality check will be performed based on the local MAC addresses. All packets that do not pass through the locality check will be dropped.

This LFB can optionally participate in Ethernet flow control in cooperation with EtherMACOut LFB. This document does not go into the details of how this is implemented. This document also does not describe how the buffers that induce the flow control messages behave -- it is assumed that such artifacts exist, and describing them is out of scope in this document.

\subsubsection{Components}

The Adminstatus component is defined for the CE to administratively manage the status of the LFB. The CE may administratively start up or shut down the LFB by changing the value of Adminstatus. The default value is set to 'Down'.

The LocalMACAddresses component specifies the local MAC addresses based on which locality checks will be made. This component is an array of MAC addresses and of 'read-write' access permission.

An L2BridgingPathEnable component captures whether the LFB is set to work as an L2 bridge. An FE that does not support bridging will internally set this flag to false and additionally set the flag property as read-only. The default value for the component is 'false'.

The PromiscuousMode component specifies whether the LFB is set to work in a promiscuous mode. The default value for the component is 'false'. 
The TxFlowControl component defines whether the LFB is performing flow control on sending packets. The default value is 'false'. Note that the component is defined as "optional". If an FE does not implement the component while a CE tries to configure the component to that $\mathrm{FE}$, an error from the $\mathrm{FE}$ may be responded to the $\mathrm{CE}$ with an error code like 0x09 (E_COMPONENT_DOES_NOT_EXIST) or 0x15 (E_NOT_SUPPORTED), depending on the FE processing. See [RFC5810] for details.

The RxFlowControl component defines whether the LFB is performing flow control on receiving packets. The default value is 'false'. The component is defined as "optional".

A struct component, MACInStats, defines a set of statistics for this LFB, including the number of received packets and the number of dropped packets. Note that this statistics component is optional to implementers. If a CE tries to query the component while it is not implemented in an $\mathrm{FE}$, an error code will be responded to the $\mathrm{CE}$ indicating the error type like 0x09 (E_COMPONENT_DOES_NOT_EXIST) or Ox15 (E_NOT_SUPPORTED), depending on the FE implementation.

\subsubsection{Capabilities}

This LFB does not have a list of capabilities.

\subsubsection{Events}

This LFB does not have any events specified.

\subsubsection{Etherclassifier}

The EtherClassifier LFB abstracts the process to decapsulate Ethernet packets and then classify them.

\subsubsection{Data Handling}

This LFB describes the process of decapsulating Ethernet packets and classifying them into various network-layer data packets according to information included in the Ethernet packets headers.

The LFB is expected to receive all types of Ethernet packets (via a singleton input known as "EtherPktsIn"), which are usually output from an upstream LFB like EtherMACIn LFB. This input is also capable of multiplexing to allow for multiple upstream LFBs to be connected. For instance, when an L2 bridging function is enabled in the EtherMACIn LFB, some L2 bridging LFBs may be applied. In this case, after L2 processing, some Ethernet packets may have to be input to the EtherClassifier LFB for classification, while simultaneously, 
packets directly output from EtherMACIn may also need to input to this LFB. This input is capable of handling such a case. Usually, all expected Ethernet packets will be associated with a PHYPortID metadata, indicating the physical port from which the packet comes. In some cases, for instance, in a MACinMAC case, a LogicalPortID metadata may be expected to associate with the Ethernet packet to further indicate the logical port to which the Ethernet packet belongs. Note that PHYPortID metadata is always expected while LogicalPortID metadata is optionally expected.

Two output LFB ports are defined.

The first output is a group output port known as "ClassifyOut". Types of network-layer protocol packets are output to instances of the port group. Because there may be various types of protocol packets at the output ports, the produced output frame is defined as arbitrary for the purpose of wide extensibility in the future. Metadata to be carried along with the packet data is produced at this LFB for consumption by downstream LFBs. The metadata passed downstream includes PHYPortID, as well as information on Ethernet type, source MAC address, destination MAC address, and the logical port ID. If the original packet is a VLAN packet and contains a VLAN ID and a VLAN priority value, then the VLAN ID and the VLAN priority value are also carried downstream as metadata. As a result, the VLAN ID and priority metadata are defined with the availability of "conditional".

The second output is a singleton output port known as "Exceptionout", which will output packets for which the data processing failed, along with an additional ExceptionID metadata to indicate what caused the exception. Currently defined exception types include:

o There is no matching when classifying the packet.

Usually, the Exceptionout port may point to nowhere, indicating packets with exceptions are dropped, while in some cases, the output may be pointed to the path to the CE for further processing, depending on individual implementations.

\subsubsection{Components}

An EtherDispatchTable array component is defined in the LFB to dispatch every Ethernet packet to the output group according to the logical port ID assigned by the VlanInputTable to the packet and the Ethernet type in the Ethernet packet header. Each row of the array is a struct containing a logical port ID, an EtherType and an output index. With the CE configuring the dispatch table, the LFB can be expected to classify various network-layer protocol type packets and 
output them at different output ports. It is expected that the LFB classify packets according to protocols like IPv4, IPv6, MPLS, Address Resolution Protocol (ARP), Neighbor Discovery (ND), etc.

A VlanInputTable array component is defined in the LFB to classify VLAN Ethernet packets. Each row of the array is a struct containing an incoming port ID, a VLAN ID, and a logical port ID. According to IEEE VLAN specifications, all Ethernet packets can be recognized as VLAN types by defining that if there is no VLAN encapsulation in a packet, a case with VLAN tag 0 is considered. Every input packet is assigned with a new LogicalPortID according to the packet's incoming port ID and the VLAN ID. A packet's incoming port ID is defined as a logical port ID if a logical port ID is associated with the packet or a physical port ID if no logical port ID is associated. The VLAN ID is exactly the VLAN ID in the packet if it is a VLAN packet, or 0 if it is not. Note that a logical port ID of a packet may be rewritten with a new one by the VlanInputTable processing.

Note that the logical port ID and physical port ID mentioned above are all originally configured by the $\mathrm{CE}$, and are globally effective within a ForCES NE (Network Element). To distinguish a physical port ID from a logical port ID in the incoming port ID field of the VlanInputTable, physical port ID and logical port ID must be assigned with separate number spaces.

An array component, Etherclassifystats, defines a set of statistics for this LFB, measuring the number of packets per EtherType. Each row of the array is a struct containing an EtherType and a packet number. Note that this statistics component is optional to implementers.

\subsubsection{Capabilities}

This LFB does not have a list of capabilities.

5.1.3.4. Events

This LFB has no events specified.

5.1.4. EtherEncap

The EtherEncap LFB abstracts the process to replace or attach appropriate Ethernet headers to the packet.

5.1.4.1. Data Handling

This LFB abstracts the process of encapsulating Ethernet headers onto received packets. The encapsulation is based on passed metadata. 
The LFB is expected to receive IPV4 and IPV6 packets (via a singleton input port known as "EncapIn"), which may be connected to an upstream LFB like IPv4NextHop, IPv6NextHop, BasicMetadataDispatch, or any LFB that requires output packets for Ethernet encapsulation. The LFB always expects from upstream LFBs the MediaEncapInfoIndex metadata, which is used as a search key to look up the encapsulation table Encaptable by the search key matching the table index. An input packet may also optionally receive a VLAN priority metadata, indicating that the packet originally had a priority value. The priority value will be loaded back to the packet when encapsulating. The optional VLAN priority metadata is defined with a default value of 0 .

Two singleton output LFB ports are defined.

The first singleton output is known as "Successout". Upon a successful table lookup, the destination and source MAC addresses and the logical media port (L2PortID) are found in the matching table entry. The CE may set the VlanID in case VLANs are used. By default, the table entry for VlanID of 0 is used as per IEEE rules [IEEE.802-1Q]. Whatever the value of VlanID, if the input metadata VlanPriority is non-zero, the packet will have a VLAN tag. If the VlanPriority and the VlanID are all zero, there is no VLAN tag for this packet. After replacing or attaching the appropriate Ethernet headers to the packet is complete, the packet is passed out on the "Successout" LFB port to a downstream LFB instance along with the L2PortID.

The second singleton output is known as "Exceptionout" and will output packets for which the table lookup fails, along with an additional ExceptionID metadata. Currently defined exception types only include the following cases:

- The MediaEncapInfoIndex value of the packet is invalid and can not be allocated in the EncapTable.

- The packet failed lookup of the EncapTable table even though the MediaEncapInfoIndex is valid.

The upstream LFB may be programmed by the CE to pass along a MediaEncapInfoIndex that does not exist in the Encaptable. This allows for resolution of the $L 2$ headers, if needed, to be made at the L2 encapsulation level, in this case, Ethernet via ARP or ND (or other methods depending on the link-layer technology), when a table miss occurs.

For neighbor L2 header resolution (table miss exception), the processing LFB may pass this packet to the CE via the redirect LFB or 
FE software or another LFB instance for further resolution. In such a case, the metadata NextHopIPv4Addr or NextHopIPv6Addr generated by the next-hop LFB is also passed to the exception handling. Such an IP address could be used to do activities such as ARP or ND by the handler to which it is passed.

The result of the L2 resolution is to update the EncapTable as well as the next-hop LFB so subsequent packets do not fail Encaptable lookup. The EtherEncap LFB does not make any assumptions of how the EncapTable is updated by the CE (or whether ARP/ND is used dynamically or static maps exist).

Downstream LFB instances could be either an EtherMACOut type or a BasicMetadataDispatch type. If the final packet L2 processing is on a per-media-port basis, resides on a different FE, or needs L2 header resolution, then it makes sense for the model to use a BasicMetadataDispatch LFB to fan out to different LFB instances. If there is a direct egress port point, then it makes sense for the model to have a downstream LFB instance be an EtherMACOut.

\subsubsection{Components}

This LFB has only one component named Encaptable, which is defined as an array. Each row of the array is a struct containing the destination MAC address, the source MAC address, the VLAN ID with a default value of zero, and the output logical L2 port ID.

\subsubsection{Capabilities}

This LFB does not have a list of capabilities.

5.1.4.4. Events

This LFB does not have any events specified.

\subsubsection{EtherMACOut}

The EtherMACOut LFB abstracts an Ethernet port at the MAC data link layer. This LFB describes Ethernet packet output process. Ethernet output functions are closely related to Ethernet input functions; therefore, many components defined in this LFB are aliases of EtherMACIn LFB components. 


\subsubsection{Data Handling}

The LFB is expected to receive all types of Ethernet packets (via a singleton input known as "EtherPktsIn"), which are usually output from an Ethernet encapsulation LFB along with a metadata indicating the ID of the physical port that the packet will go through.

The LFB is defined with a singleton output port known as "EtherPktsOut". All output packets are in Ethernet format, possibly with various Ethernet types, along with a metadata indicating the ID of the physical port that the packet is to go through. This output links to a downstream LFB that is usually an Ethernet physical LFB like the EtherPHYCop LFB.

This LFB can optionally participate in Ethernet flow control in cooperation with the EtherMACIn LFB. This document does not go into the details of how this is implemented. This document also does not describe how the buffers that induce the flow control messages behave -- it is assumed that such artifacts exist, but describing them is out of the scope of this document.

Note that as a base definition, functions like multiple virtual MAC layers are not supported in this LFB version. It may be supported in the future by defining a subclass or a new version of this LFB.

\subsubsection{Components}

The Adminstatus component is defined for the CE to administratively manage the status of the LFB. The CE may administratively start up or shut down the LFB by changing the value of Adminstatus. The default value is set to 'Down'. Note that this component is defined as an alias of the Adminstatus component in the EtherMACIn LFB. This infers that an EtherMACOut LFB usually coexists with an EtherMACIn LFB, both of which share the same administrative status management by the CE. Alias properties, as defined in the ForCES FE model [RFC5812], will be used by the CE to declare the target component to which the alias refers, which includes the target LFB class and instance IDs as well as the path to the target component.

The MTU component defines the maximum transmission unit.

The optional TxFlowControl component defines whether or not the LFB is performing flow control on sending packets. The default value is 'false'. Note that this component is defined as an alias of the TxFlowControl component in the EtherMACIn LFB.

The optional RxFlowControl component defines whether or not the LFB is performing flow control on receiving packets. The default value 
is 'false'. Note that this component is defined as an alias of the RxFlowControl component in the EtherMACIn LFB.

A struct component, MACOutstats, defines a set of statistics for this LFB, including the number of transmitted packets and the number of dropped packets. This statistics component is optional to implementers.

\subsubsection{Capabilities}

This LFB does not have a list of capabilities.

5.1.5.4. Events

This LFB does not have any events specified.

\subsection{IP Packet Validation LFBS}

The LFBs are defined to abstract the IP packet validation process. An IPv4Validator LFB is specifically for IPv4 protocol validation, and an IPv6Validator LFB is specifically for IPv6.

5.2.1. IPv4Validator

The IPv4Validator LFB performs IPv4 packet validation.

\subsubsection{Data Handling}

This LFB performs IPv4 validation according to [RFC1812] and its updates. The IPv4 packet will be output to the corresponding LFB port, indicating whether the packet is unicast or multicast or whether an exception has occurred or the validation failed.

This LFB always expects, as input, packets that have been indicated as IPv4 packets by an upstream LFB, like an EtherClassifier LFB. There is no specific metadata expected by the input of the LFB.

Four output LFB ports are defined.

All validated IPv4 unicast packets will be output at the singleton port known as "IPv4Unicastout". All validated IPv4 multicast packets will be output at the singleton port known as "IPv4Multicastout" port.

A singleton port known as "Exceptionout" is defined to output packets that have been validated as exception packets. An exception ID metadata is produced to indicate what has caused the exception. An exception case is the case when a packet needs further processing 
before being normally forwarded. Currently defined exception types include:

- Packet with expired TTL

- Packet with header length more than 5 words

- Packet IP head including router alert options

- Packet with exceptional source address

- Packet with exceptional destination address

Note that although Time to Live (TTL) is checked in this LFB for validity, operations like TTL decrement are made by the downstream forwarding LFB.

The final singleton port known as "Failout" is defined for all packets that have errors and failed the validation process. An error case is when a packet is unable to be further processed or forwarded without being dropped. An error ID is associated with a packet to indicate the failure reason. Currently defined failure reasons include:

- Packet with size reported less than 20 bytes

- Packet with version not IPv4

o Packet with header length less than 5 words

- Packet with total length field less than 20 bytes

- Packet with invalid checksum

- Packet with invalid source address

- Packet with invalid destination address

\subsubsection{Components}

This LFB has only one struct component, the IPv4ValidatorStatisticsType, which defines a set of statistics for validation process, including the number of bad header packets, the number of bad total length packets, the number of bad TTL packets, and the number of bad checksum packets. This statistics component is optional to implementers. 


\subsubsection{Capabilities}

This LFB does not have a list of capabilities

\subsubsection{Events}

This LFB does not have any events specified.

\subsubsection{IPv6Validator}

The IPv6Validator LFB performs IPv6 packet validation.

\subsubsection{Data Handling}

This LFB performs IPv6 validation according to [RFC2460] and its updates. Then the IPv6 packet will be output to the corresponding port regarding of the validation result, indicating whether the packet is a unicast or a multicast one, an exception has occurred or the validation failed.

This LFB always expects, as input, packets that have been indicated as IPv6 packets by an upstream LFB, like an EtherClassifier LFB. There is no specific metadata expected by the input of the LFB.

Similar to the IPv4validator LFB, the IPv6Validator LFB has also defined four output ports to emit packets with various validation results.

All validated IPv6 unicast packets will be output at the singleton port known as "IPv6Unicastout". All validated IPv6 multicast packets will be output at the singleton port known as "IPv6Multicastout". There is no metadata produced at this LFB.

A singleton port known as "Exceptionout" is defined to output packets that have been validated as exception packets. An exception case is when a packet needs further processing before being normally forwarded. An exception ID metadata is produced to indicate what caused the exception. Currently defined exception types include:

- Packet with hop limit to zero

o Packet with next header set to hop-by-hop

- Packet with exceptional source address

- Packet with exceptional destination address 
The final singleton port known as "Failout" is defined for all packets that have errors and failed the validation process. An error case when a packet is unable to be further processed or forwarded without being dropped. A validate error ID is associated to every failed packet to indicate the reason. Currently defined reasons include:

- Packet with size reported less than 40 bytes

- Packet with version not IPv6

- Packet with invalid source address

- Packet with invalid destination address

Note that in the base type library, definitions for exception ID and validate error ID metadata are applied to both IPv4Validator and IPv6Validator LFBs, i.e., the two LFBs share the same metadata definition, with different ID assignment inside.

\subsubsection{Components}

This LFB has only one struct component, the IPv6ValidatorstatisticsType, which defines a set of statistics for the validation process, including the number of bad header packets, the number of bad total length packets, and the number of bad hop limit packets. Note that this component is optional to implementers.

\subsubsection{Capabilities}

This LFB does not have a list of capabilities.

\subsubsection{Events}

This LFB does not have any events specified.

\subsection{IP Forwarding LFBS}

IP Forwarding LFBs are specifically defined to abstract the IP forwarding processes. As definitions for a base LFB library, this document restricts its LFB definition scope only to IP unicast forwarding. IP multicast may be defined in future documents.

The two fundamental tasks performed in IP unicast forwarding constitute looking up the forwarding information table to find nexthop information and then using the resulting next-hop details to forward packets out on specific physical output ports. This document models the forwarding processes by abstracting out the described two 
steps. Whereas this document describes functional LFB models that are modular, there may be multiple ways to implement the abstracted models. It is not intended or expected that the provided LFB models constrain implementations.

Based on the IP forwarding abstraction, two kinds of typical IP unicast forwarding LFBS are defined: unicast LPM lookup LFB and nexthop application LFB. They are further distinguished by IPV4 and IPV6 protocols.

\subsubsection{IPv4UcastLPM}

The IPv4UcastLPM LFB abstracts the IPv4 unicast Longest Prefix Match (LPM) process.

This LFB also provides facilities to support users to implement equal-cost multipath (ECMP) routing or reverse path forwarding (RPF). However, this LFB itself does not provide ECMP or RPF. To fully implement ECMP or RPF, additional specific LFBs, like a specific ECMP LFB or an RPF LFB, will have to be defined.

\subsubsection{Data Handling}

This LFB performs the IPv4 unicast LPM table lookup. It always expects as input IPv4 unicast packets from one singleton input known as "PktsIn". Then, the LFB uses the destination IPv4 address of every packet as a search key to look up the IPv4 prefix table and generate a hop selector as the matching result. The hop selector is passed as packet metadata to downstream LFBs and will usually be used there as a search index to find more next-hop information.

Three singleton output LFB ports are defined.

The first singleton output is known as "Normalout" and outputs IPv4 unicast packets that succeed the LPM lookup (and got a hop selector). The hop selector is associated with the packet as a metadata. Downstream from the LPM LFB is usually a next-hop application LFB, like an IPv4NextHop LFB.

The second singleton output is known as "ECMPOut" and is defined to provide support for users wishing to implement ECMP.

An ECMP flag is defined in the LPM table to enable the LFB to support ECMP. When a table entry is created with the flag set to true, it indicates this table entry is for ECMP only. A packet that has passed through this prefix lookup will always output from the "ECMPOut" output port, with the hop selector being its lookup result. The output will usually go directly to a downstream ECMP processing 
LFB, where the hop selector can usually further generate optimized one or multiple next-hop routes by use of ECMP algorithms.

A default route flag is defined in the LPM table to enable the LFB to support a default route as well as loose RPF. When this flag is set to true, the table entry is identified as a default route, which also implies that the route is forbidden for RPF. If a user wants to implement RPF on FE, a specific RPF LFB will have to be defined. In such an RPF LFB, a component can be defined as an alias of the prefix table component of this LFB, as described below.

The final singleton output is known as "Exceptionout" of the IPv4UcastLPM LFB and is defined to output exception packets after the LFB processing, along with an ExceptionID metadata to indicate what caused the exception. Currently defined exception types include:

- The packet failed the LPM lookup of the prefix table.

The upstream LFB of this LFB is usually an IPv4Validator LFB. If RPF is to be adopted, the upstream can be an RPF LFB, when defined.

The downstream LFB is usually an IPv4NextHop LFB. If ECMP is adopted, the downstream can be an ECMP LFB, when defined.

\subsubsection{Components}

This LFB has two components.

The IPv4PrefixTable component is defined as an array component of the LFB. Each row of the array contains an IPv4 address, a prefix length, a hop selector, an ECMP flag and a default route flag. The LFB uses the destination IPv4 address of every input packet as a search key to look up this table in order extract a next-hop selector. The ECMP flag is for the LFB to support ECMP. The default route flag is for the LFB to support a default route and for loose $\mathrm{RPF}$.

The IPv4UcastLPMStats component is a struct component that collects statistics information, including the total number of input packets received, the IPV4 packets forwarded by this LFB, and the number of IP datagrams discarded due to no route found. Note that this component is defined as optional to implementers.

\subsubsection{Capabilities}

This LFB does not have a list of capabilities. 


\subsubsection{Events}

This LFB does not have any events specified.

\subsubsection{IPv4Next Hop}

This LFB abstracts the process of selecting IPv4 next-hop action.

\subsubsection{Data Handling}

The LFB abstracts the process of next-hop information application to IPv4 packets. It receives an IPv4 packet with an associated next-hop identifier (Hopselector) and uses the identifier as a table index to look up a next-hop table to find an appropriate LFB output port.

The LFB is expected to receive unicast IPv4 packets, via a singleton input known as "PktsIn", along with a Hopselector metadata, which is used as a table index to look up the NextHop table. The data processing involves the forwarding TTL decrement and IP checksum recalculation.

Two output LFB ports are defined.

The first output is a group output port known as "SuccessOut". On successful data processing, the packet is sent out from an LFB port from within the LFB port group as selected by the LFBOutputselectIndex value of the matched table entry. The packet is sent to a downstream LFB along with the L3PortID and MediaEncapInfoIndex metadata.

The second output is a singleton output port known as "Exceptionout", which will output packets for which the data processing failed, along with an additional ExceptionID metadata to indicate what caused the exception. Currently defined exception types include:

- The Hopselector for the packet is invalid.

- The packet failed lookup of the next-hop table even though the Hopselector is valid.

- The MTU for outgoing interface is less than the packet size.

Downstream LFB instances could be either a BasicMetadataDispatch type (Section 5.5.1), used to fan out to different LFB instances or a media-encapsulation-related type, such as an EtherEncap type or a Redirectout type (Section 5.4.2). For example, if there are Ethernet and other tunnel encapsulation, then a BasicMetadataDispatch LFB can 
use the L3PortID metadata (Section 5.3.2.2) to dispatch packets to a different encapsulator.

\subsubsection{Components}

This LFB has only one component, IPv4NextHopTable, which is defined as an array. The Hopselector received is used to match the array index of IPv4NextHopTable to find out a row of the table as the nexthop information result. Each row of the array is a struct containing:

- The L3PortID, which is the ID of the logical output port that is passed on to the downstream LFB instance. This ID indicates what kind of encapsulating port the neighbor is to use. This is L3derived information that affects L2 processing and so needs to be based from one LFB to another as metadata. Usually, this ID is used for the next-hop LFB to distinguish packets that need different L2 encapsulating. For instance, some packets may require general Ethernet encapsulation while others may require various types of tunnel encapsulations. In such a case, different L3PortIDs are assigned to the packets and are passed as metadata to a downstream LFB. A BasicMetadataDispatch LFB (Section 5.5.1) may have to be applied as the downstream LFB so as to dispatch packets to different encapsulation LFB instances according to the L3Portids.

- MTU, the Maximum Transmission Unit for the outgoing port.

o NextHopIPAddr, the IPv4 next-hop address.

- MediaEncapInfoIndex, the index that passes on to the downstream encapsulation LFB instance and that is used there as a search key to look up a table (typically media-encapsulation-related) for further encapsulation information. The search key looks up the table by matching the table index. Note that the encapsulation LFB instance that uses this metadata may not be the LFB instance that immediately follows this LFB instance in the processing. The MediaEncapInfoIndex metadata is attached here and is passed through intermediate LFBs until it is used by the encapsulation LFB instance. In some cases, depending on implementation, the CE may set the MediaEncapInfoIndex passed downstream to a value that will fail lookup when it gets to a target encapsulation LFB; such a lookup failure at that point is an indication that further resolution is needed. For an example of this approach, refer to Section 7.2, which discusses ARP and mentions this approach. 
- LFBOutputselectIndex, the LFB group output port index to select the downstream LFB port. This value identifies the specific port within the successout port group out of which packets that successfully use this next-hop entry are to be sent.

\subsubsection{Capabilities}

This LFB does not have a list of capabilities.

5.3.2.4. Events

This LFB does not have any events specified.

5.3.3. IPv6UcastLPM

The IPv6UcastLPM LFB abstracts the IPv6 unicast Longest Prefix Match (LPM) process. The definition of this LFB is similar to the IPV4UcastLPM LFB except that all IP addresses refer to IPV6 addresses.

This LFB also provides facilities to support users to implement equal-cost multipath (ECMP) routing or reverse path forwarding (RPF). However, this LFB itself does not provide ECMP or RPF. To fully implement ECMP or RPF, additional specific LFBs, like a specific ECMP LFB or an RPF LFB, will have to be defined. This work may be done in future versions of this document.

5.3.3.1. Data Handling

This LFB performs the IPv6 unicast LPM table lookup. It always expects as input IPv6 unicast packets from one singleton input known as "PktsIn". The destination IPV6 address of an incoming packet is used as a search key to look up the IPv6 prefix table and generate a hop selector. This hop selector result is associated to the packet as a metadata and sent to downstream LFBs; it will usually be used in downstream LFBs as a search key to find more next-hop information.

Three singleton output LFB ports are defined.

The first singleton output is known as "Normalout" and outputs IPv6 unicast packets that succeed the LPM lookup (and got a hop selector). The hop selector is associated with the packet as a metadata. Downstream from the LPM LFB is usually a next-hop application LFB, like an IPv6NextHop LFB.

The second singleton output is known as "ECMPOut" and is defined to provide support for users wishing to implement ECMP. 
An ECMP flag is defined in the LPM table to enable the LFB to support ECMP. When a table entry is created with the flag set to true, it indicates this table entry is for ECMP only. A packet that has passed through this prefix lookup will always output from the "ECMPOut" output port, with the hop selector being its lookup result. The output will usually go directly to a downstream ECMP processing LFB, where the hop selector can usually further generate optimized one or multiple next-hop routes by use of ECMP algorithms.

A default route flag is defined in the LPM table to enable the LFB to support a default route as well as loose RPF. When this flag is set to true, the table entry is identified as a default route, which also implies that the route is forbidden for RPF.

If a user wants to implement RPF on FE, a specific RPF LFB will have to be defined. In such an RPF LFB, a component can be defined as an alias of the prefix table component of this LFB, as described below.

The final singleton output is known as "Exceptionout" of the IPV6UcastLPM LFB and is defined to output exception packets after the LFB processing, along with an ExceptionID metadata to indicate what caused the exception. Currently defined exception types include:

o The packet failed the LPM lookup of the prefix table.

The upstream LFB of this LFB is usually an IPv6Validator LFB. If RPF is to be adopted, the upstream can be an RPF LFB, when defined.

The downstream LFB is usually an IPv6NextHop LFB. If ECMP is adopted, the downstream can be an ECMP LFB, when defined.

\subsubsection{Components}

This LFB has two components.

The IPv6PrefixTable component is defined as an array component of the LFB. Each row of the array contains an IPv6 address, a prefix length, a hop selector, an ECMP flag, and a default route flag. The ECMP flag is so the LFB can support ECMP. The default route flag is for the LFB to support a default route and for loose RPF, as described earlier.

The IPv6UcastLPMStats component is a struct component that collects statistics information, including the total number of input packets received, the IPv6 packets forwarded by this LFB and the number of IP datagrams discarded due to no route found. Note that the component is defined as optional to implementers. 


\subsubsection{Capabilities}

This LFB does not have a list of capabilities.

\subsubsection{Events}

This LFB does not have any events specified.

\subsubsection{IPv6NextHop}

This LFB abstracts the process of selecting IPv6 next-hop action.

\subsubsection{Data Handling}

The LFB abstracts the process of next-hop information application to IPv6 packets. It receives an IPv6 packet with an associated next-hop identifier (Hopselector) and uses the identifier to look up a nexthop table to find an appropriate output port from the LFB.

The LFB is expected to receive unicast IPv6 packets, via a singleton input known as "PktsIn", along with a Hopselector metadata, which is used as a table index to look up the next-hop table.

Two output LFB ports are defined.

The first output is a group output port known as "Successout". On successful data processing, the packet is sent out from an LFB port from within the LFB port group as selected by the LFBOutputselectIndex value of the matched table entry. The packet is sent to a downstream LFB along with the L3PortID and MediaEncapInfoIndex metadata.

The second output is a singleton output port known as "Exceptionout", which will output packets for which the data processing failed, along with an additional ExceptionID metadata to indicate what caused the exception. Currently defined exception types include:

- The Hopselector for the packet is invalid.

- The packet failed lookup of the next-hop table even though the Hopselector is valid.

- The MTU for outgoing interface is less than the packet size.

Downstream LFB instances could be either a BasicMetadataDispatch type, used to fan out to different LFB instances, or a media encapsulation related type, such as an EtherEncap type or a Redirectout type. For example, when the downstream LFB is 
BasicMetadataDispatch and Ethernet and other tunnel encapsulation exist downstream from BasicMetadataDispatch, then the BasicMetadataDispatch LFB can use the L3PortID metadata (see section below) to dispatch packets to the different encapsulator LFBs.

\subsubsection{Components}

This LFB has only one component named IPv6NextHopTable, which is defined as an array. The array index of IPv6NextHopTable is used for a Hopselector to find out a row of the table as the next-hop information. Each row of the array is a struct containing:

- The L3PortID, which is the ID of the logical output port that is passed onto the downstream LFB instance. This ID indicates what kind of encapsulating port the neighbor is to use. This is L3derived information that affects L2 processing and so needs to be based from one LFB to another as metadata. Usually, this ID is used for the next-hop LFB to distinguish packets that need different L2 encapsulating. For instance, some packets may require general Ethernet encapsulation while others may require various types of tunnel encapsulations. In such a case, different L3PortIDs are assigned to the packets and are passed as metadata to a downstream LFB. A BasicMetadataDispatch LFB (Section 5.5.1) may have to be applied as the downstream LFB so as to dispatch packets to different encapsulation LFB instances according to the L3Portids.

- MTU, the Maximum Transmission Unit for the outgoing port.

o NextHopIPAddr, the IPv6 next-hop address.

- MediaEncapInfoIndex, the index that is passed on to the downstream encapsulation LFB instance and that is used there as a search key to look up a table (typically media-encapsulation-related) for further encapsulation information. The search key looks up the table by matching the table index. Note that the encapsulation LFB instance that uses this metadata may not be the LFB instance that immediately follows this LFB instance in the processing. The MediaEncapInfoIndex metadata is attached here and is passed through intermediate LFBs until it is used by the encapsulation LFB instance. In some cases, depending on implementation, the CE may set the MediaEncapInfoIndex passed downstream to a value that will fail lookup when it gets to a target encapsulation LFB; such a lookup failure at that point is an indication that further resolution is needed. For an example of this approach, refer to Section 7.2, which discusses ARP and mentions this approach. 
- LFBOutputselectIndex, the LFB group output port index to select the downstream LFB port. This value identifies the specific port within the successout port group out of which packets that successfully use this next-hop entry are to be sent.

\subsubsection{Capabilities}

This LFB does not have a list of capabilities.

5.3.4.4. Events

This LFB does not have any events specified.

5.4. Redirect LFBS

Redirect LFBs abstract the data packet transportation process between the $\mathrm{CE}$ and $\mathrm{FE}$. Some packets output from some LFBs may have to be delivered to the $\mathrm{CE}$ for further processing, and some packets generated by the CE may have to be delivered to the FE and further to some specific LFBs for data path processing. According to [RFC5810], data packets and their associated metadata are encapsulated in a ForCES redirect message for transportation between $\mathrm{CE}$ and $\mathrm{FE}$. We define two LFBS to abstract the process: a RedirectIn LFB and a Redirectout LFB. Usually, in an LFB topology of an FE, only one RedirectIn LFB instance and one RedirectOut LFB instance exist.

5.4.1. RedirectIn

The RedirectIn LFB abstracts the process for the CE to inject data packets into the FE data path.

5.4.1.1. Data Handling

A RedirectIn LFB abstracts the process for the CE to inject data packets into the FE LFB topology so as to input data packets into FE data paths. From the LFB topology's point of view, the RedirectIn LFB acts as a source point for data packets coming from the $\mathrm{CE}$; therefore, the RedirectIn LFB is defined with a single output LFB port (and no input LFB port).

The single output port of RedirectIn LFB is defined as a group output type with the name of "Pktsout". Packets produced by this output will have arbitrary frame types decided by the $\mathrm{CE}$ that generated the packets. Possible frames may include IPv4, IPv6, or ARP protocol packets. The $\mathrm{CE}$ may associate some metadata to indicate the frame types and may also associate other metadata to indicate various information on the packets. Among them, there MUST exist a RedirectIndex metadata, which is an integer acting as an index. When 
the CE transmits the metadata along with the packet to a RedirectIn LFB, the LFB will read the RedirectIndex metadata and output the packet to one of its group output port instances, whose port index is indicated by this metadata. Any other metadata, in addition to RedirectIndex, will be passed untouched along the packet delivered by the CE to the downstream LFB. This means the RedirectIndex metadata from CE will be "consumed" by the RedirectIn LFB and will not be passed to downstream LFB. Note that a packet from the CE without a RedirectIndex metadata associated will be dropped by the LFB. Note that all metadata visible to the LFB need to be global and IANA controlled. See Section 8 ("IANA Considerations") of this document for more details about a metadata ID space that can be used by vendors and is "Reserved for Private Use".

\subsubsection{Components}

An optional statistics component is defined to collect the number of packets received by the LFB from the CE. There are no other components defined for the current version of the LFB.

\subsubsection{Capabilities}

This LFB does not have a list of capabilities.

5.4.1.4. Events

This LFB does not have any events specified.

5.4.2. Redirectout

Redirectout LFB abstracts the process for LFBs in the FE to deliver data packets to the $\mathrm{CE}$.

\subsubsection{Data Handling}

A Redirectout LFB abstracts the process for LFBs in the FE to deliver data packets to the CE. From the LFB topology's point of view, the RedirectOut LFB acts as a sink point for data packets going to the CE; therefore, the RedirectOut LFB is defined with a single input LFB port (and no output LFB port).

The Redirectout LFB has only one singleton input, known as "PktsIn", but is capable of receiving packets from multiple LFBs by multiplexing this input. The input expects any kind of frame type; therefore, the frame type has been specified as arbitrary, and also all types of metadata are expected. All associated metadata produced (but not consumed) by previous processed LFBs should be delivered to the $\mathrm{CE}$ via the ForCES protocol redirect message [RFC5810]. The CE 
can decide how to process the redirected packet by referencing the associated metadata. As an example, a packet could be redirected by the $\mathrm{FE}$ to the $\mathrm{CE}$ because the EtherEncap LFB is not able to resolve L2 information. The metadata "ExceptionID" created by the EtherEncap LFB is passed along with the packet and should be sufficient for the $\mathrm{CE}$ to do the necessary processing and resolve the L2 entry required. Note that all metadata visible to the LFB need to be global and IANA controlled. See Section 8 ("IANA Considerations") of this document for more details about a metadata ID space that can be used by vendors and is "Reserved for Private Use".

\subsubsection{Components}

An optional statistics component is defined to collect the number of packets sent by the LFB to the CE. There are no other components defined for the current version of the LFB.

\subsubsection{Capabilities}

This LFB does not have a list of capabilities.

\subsubsection{Events}

This LFB does not have any events specified.

\subsection{General Purpose LFBs}

\subsubsection{BasicMetadataDispatch}

The BasicMetadataDispatch LFB is defined to abstract the process in which a packet is dispatched to some output path based on its associated metadata value.

\subsubsection{Data Handling}

The BasicMetadataDispatch LFB has only one singleton input known as "PktsIn". Every input packet should be associated with a metadata that will be used by the LFB to do the dispatch. This LFB contains a metadata ID and a dispatch table named MetadataDispatchTable, all configured by the CE. The metadata ID specifies which metadata is to be used for dispatching packets. The MetadataDispatchTable contains entries of a metadata value and an OutputIndex, specifying that the packet with the metadata value must go out from the LFB group output port instance with the OutputIndex.

Two output LFB ports are defined. 
The first output is a group output port known as "Pktsout". A packet with its associated metadata having found an output Index by successfully looking up the dispatch table will be output to the group port instance with the corresponding index.

The second output is a singleton output port known as "Exceptionout", which will output packets for which the data processing failed, along with an additional ExceptionID metadata to indicate what caused the exception. Currently defined exception types only include one case:

- There is no matching when looking up the metadata dispatch table.

As an example, if the CE decides to dispatch packets according to a physical port ID (PHYPortID), the CE may set the ID of PHYPortID metadata to the LFB first. Moreover, the CE also sets the PHYPortID actual values (the metadata values) and assigned OutputIndex for the values to the dispatch table in the LFB. When a packet arrives, a PHYPortID metadata is found associated with the packet, and the metadata value is further used as a key to look up the dispatch table to find out an output port instance for the packet.

Currently, the BasicMetadataDispatch LFB only allows the metadata value of the dispatch table entry to be a 32-bit integer. A metadata with other value types is not supported in this version. A more complex metadata dispatch LFB may be defined in future versions of the library. In that LFB, multiple tuples of metadata with more value types supported may be used to dispatch packets.

\subsubsection{Components}

This LFB has two components. One component is MetadataID and the other is MetadataDispatchTable. Each row entry of the dispatch table is a struct containing the metadata value and the OutputIndex. Note that currently, the metadata value is only allowed to be a 32-bit integer. The metadata value is also defined as a content key for the table. The concept of content key is a searching key for tables, which is defined in the ForCES FE model [RFC5812]. With the content key, the CE can manipulate the table by means of a specific metadata value rather than by the table index only. See the ForCES FE model [RFC5812] and also the ForCES protocol [RFC5810] for more details on the definition and use of a content key.

\subsubsection{Capabilities}

This LFB does not have a list of capabilities. 


\subsubsection{Events}

This LFB does not have any events specified.

\subsubsection{GenericScheduler}

This is a preliminary generic scheduler LFB for abstracting a simple scheduling process.

\subsubsection{Data Handling}

There exist various kinds of scheduling strategies with various implementations. As a base LFB library, this document only defines a preliminary generic scheduler LFB for abstracting a simple scheduling process. Users may use this LFB as a basic LFB to further construct more complex scheduler LFBs by means of "inheritance", as described in [RFC5812].

Packets of any arbitrary frame type are received via a group input known as "PktsIn" with no additional metadata expected. This group input is capable of multiple input port instances. Each port instance may be connected to a different upstream LFB output. Inside the LFB, it is abstracted that each input port instance is connected to a queue, and the queue is marked with a queue ID whose value is exactly the same as the index of corresponding group input port instance. Scheduling disciplines are applied to all queues and also all packets in the queues. The group input port property PortGrouplimits in ObjectLFB, as defined by the ForCES FE model [RFC5810], provides means for the CE to query the capability of total queue numbers the scheduler supports. The CE can then decide how many queues it may use for a scheduling application.

Scheduled packets are output from a singleton output port of the LFB knows as "PktsOut" with no corresponding metadata.

More complex scheduler LFBs may be defined with more complex scheduling disciplines by succeeding this LFB. For instance, a priority scheduler LFB may be defined by inheriting this LFB and defining a component to indicate priorities for all input queues.

\subsubsection{Components}

The SchedulingDiscipline component is for the CE to specify a scheduling discipline to the LFB. Currently defined scheduling disciplines only include Round Robin (RR) strategy. The default scheduling discipline is thus RR. 
The Queuestats component is defined to allow the CE to query every queue status of the scheduler. It is an array component, and each row of the array is a struct containing a queue ID. Currently defined queue status includes the queue depth in packets and the queue depth in bytes. Using the queue ID as the index, the CE can query every queue for its used length in unit of packets or bytes. Note that the Queuestats component is defined as optional to implementers.

\subsubsection{Capabilities}

The following capability is currently defined for the GenericScheduler.

- The queue length limit providing the storage ability for every queue.

\subsubsection{Events}

This LFB does not have any events specified.

6. XML for LFB Library

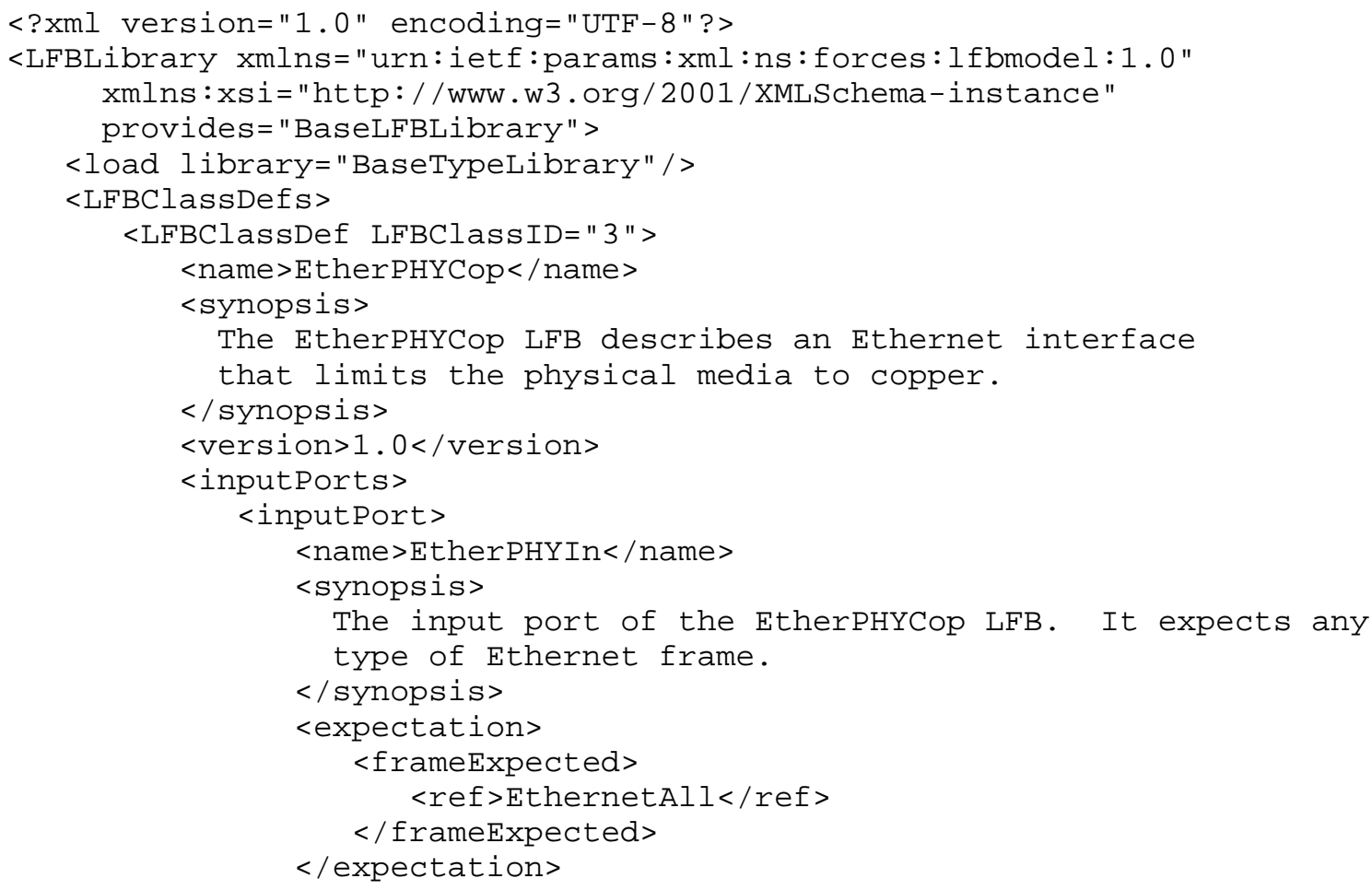




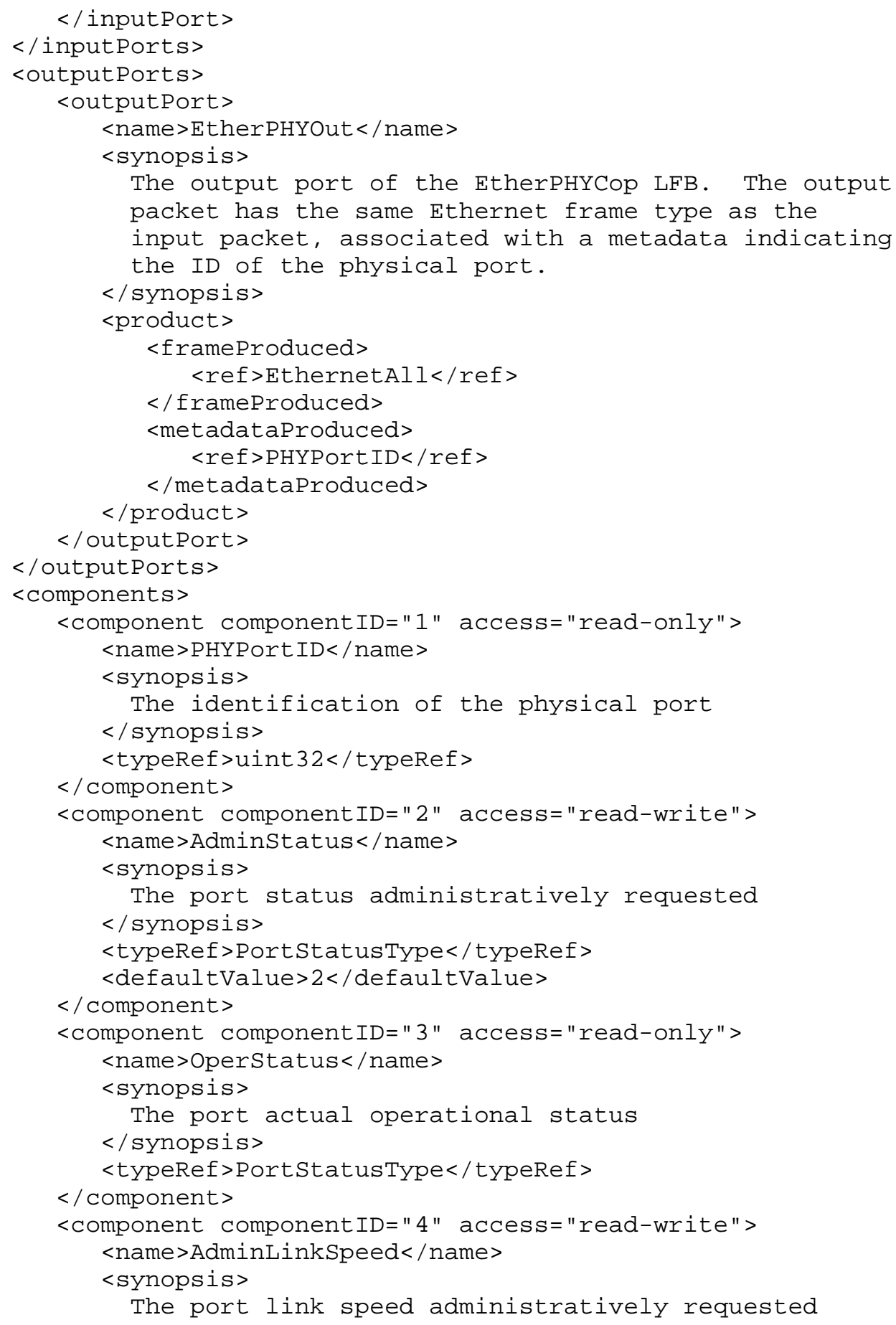




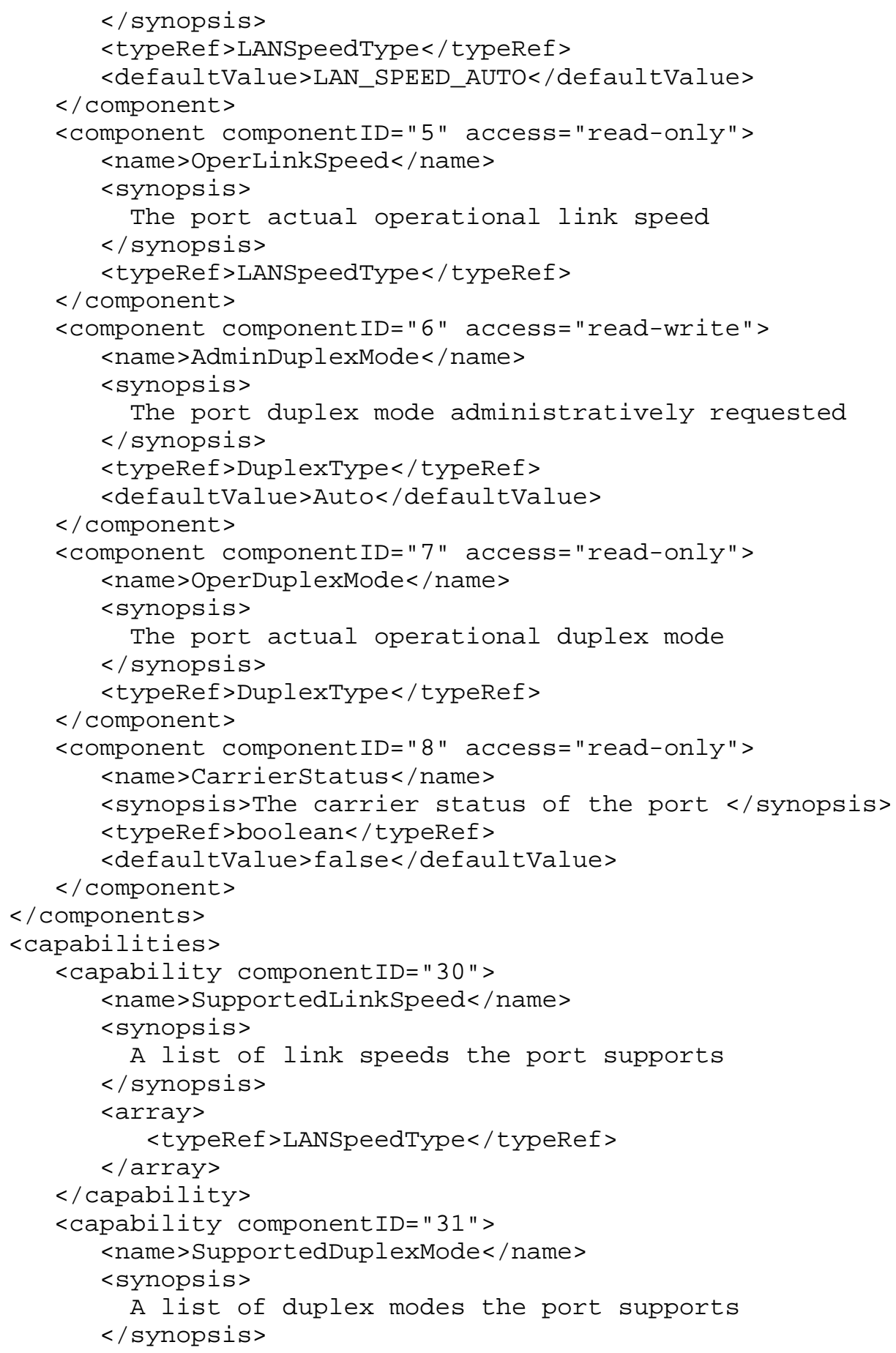




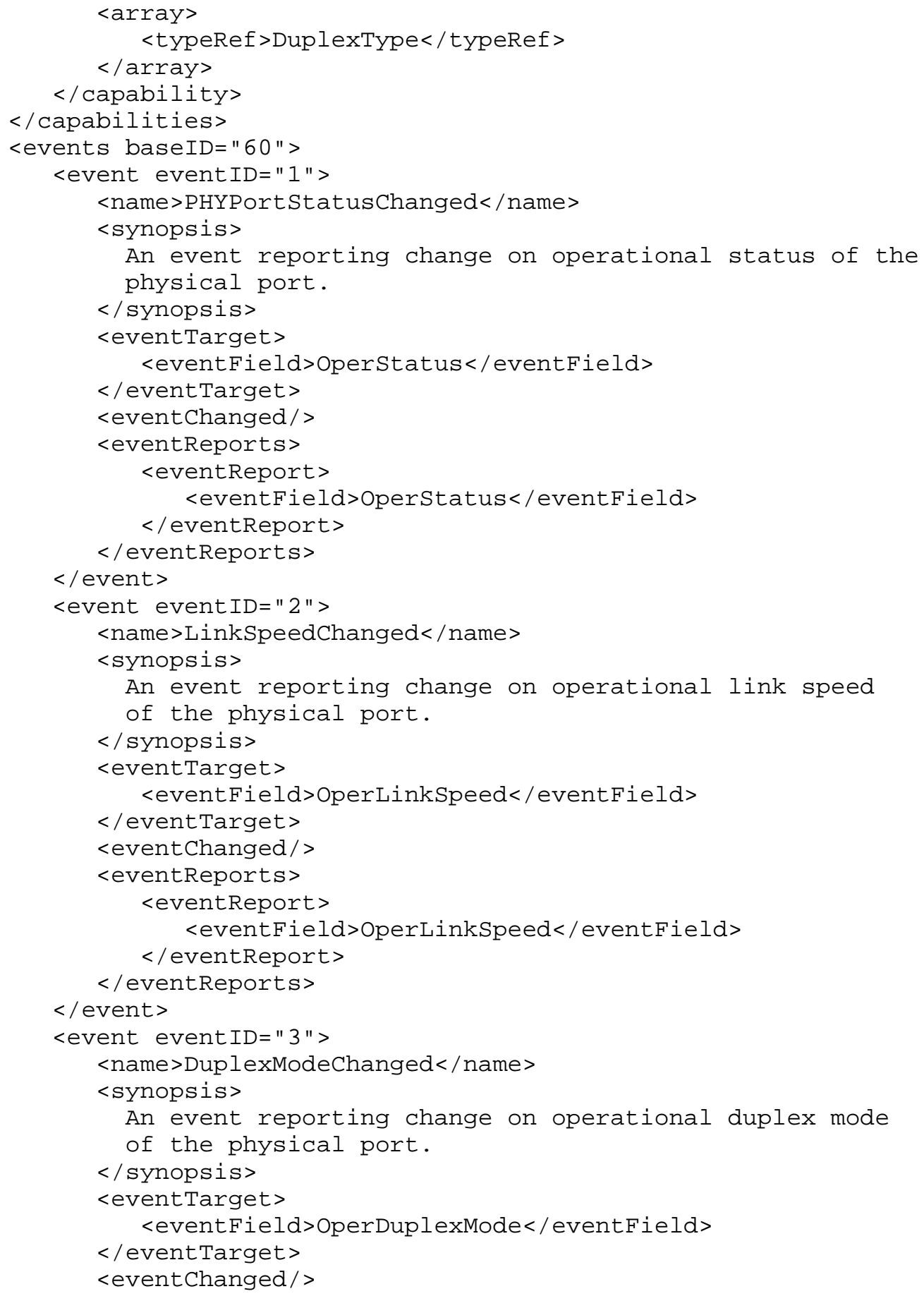

Wang, et al. 


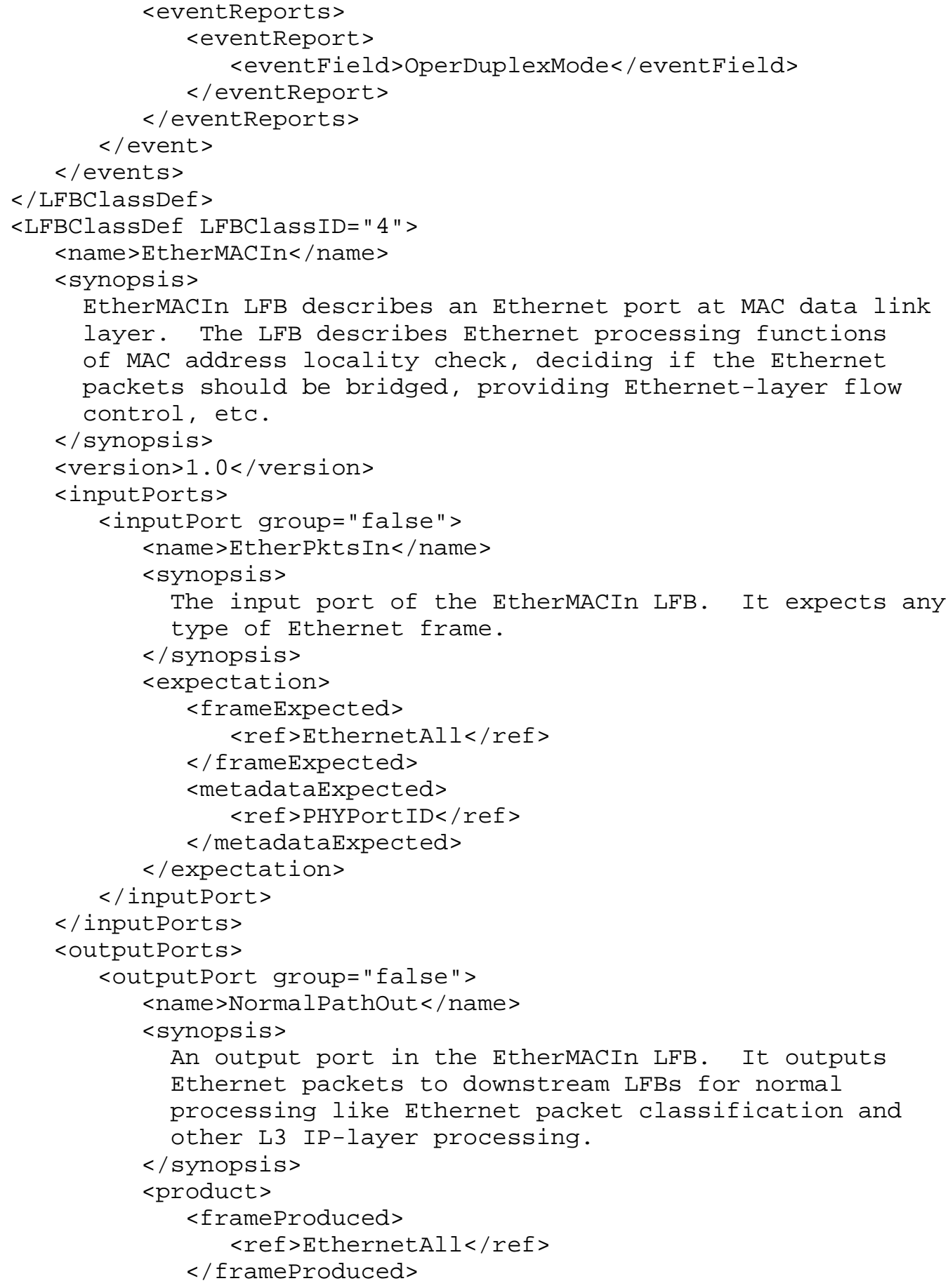

Wang, et al. 


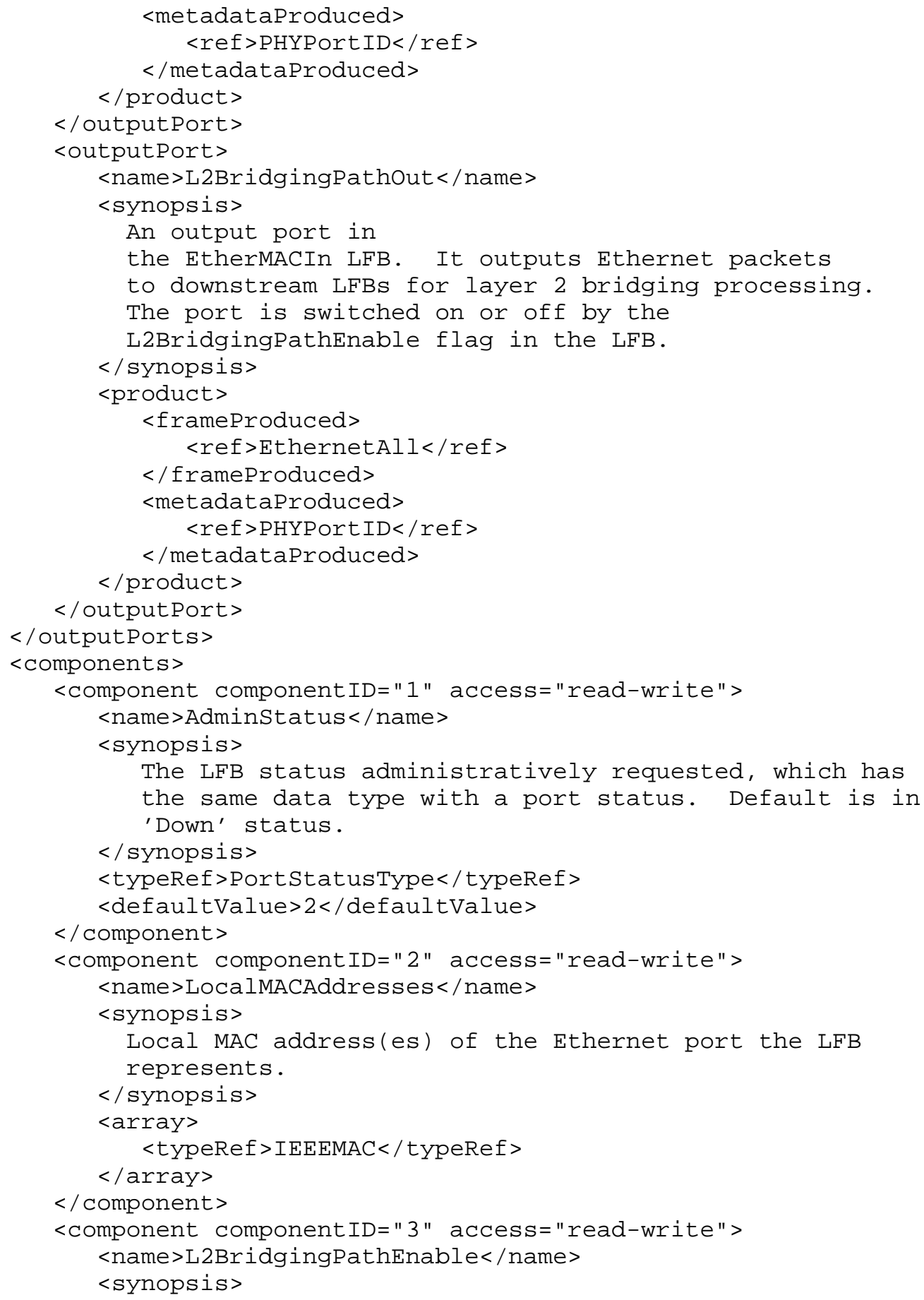

Wang, et al. 


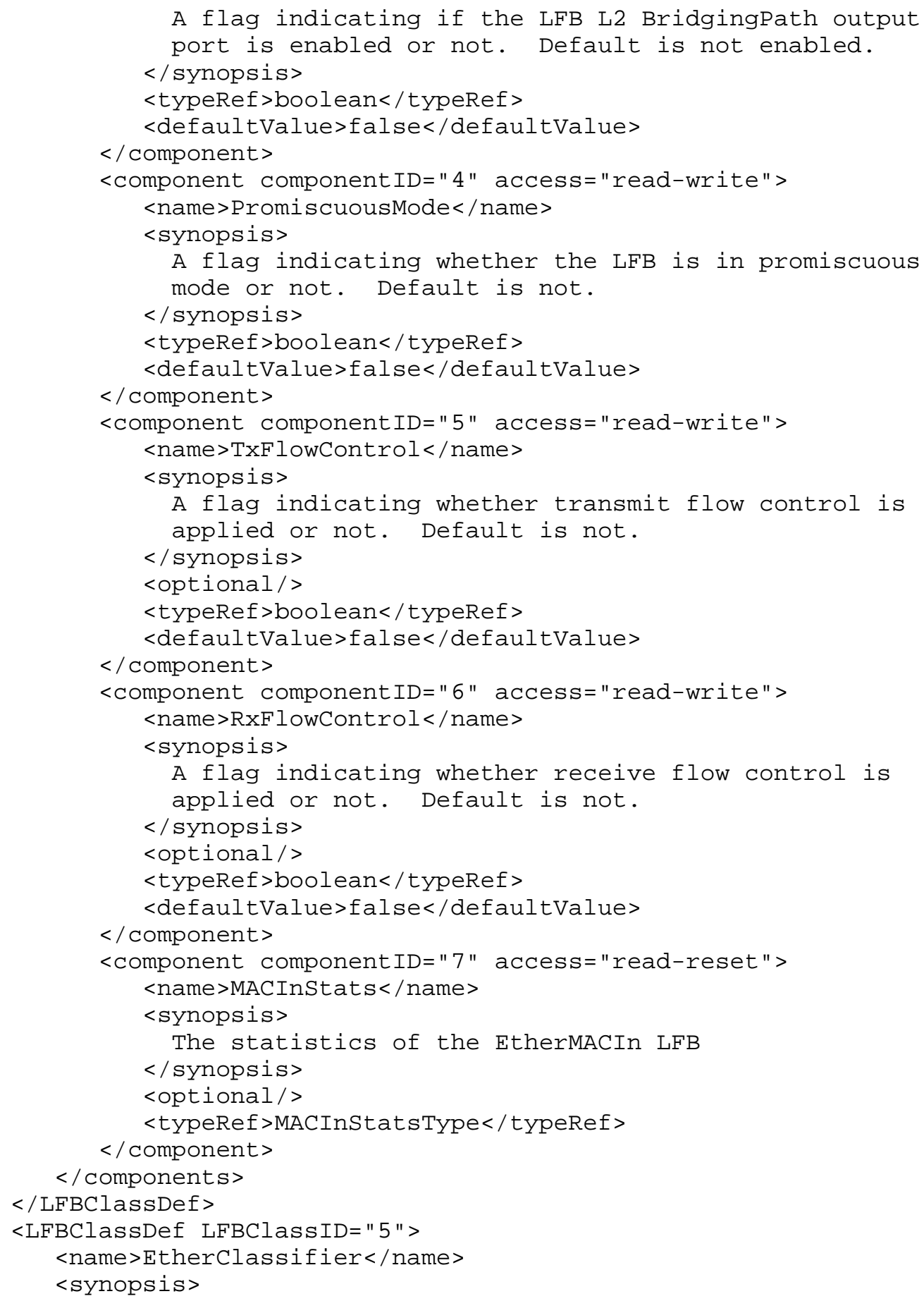




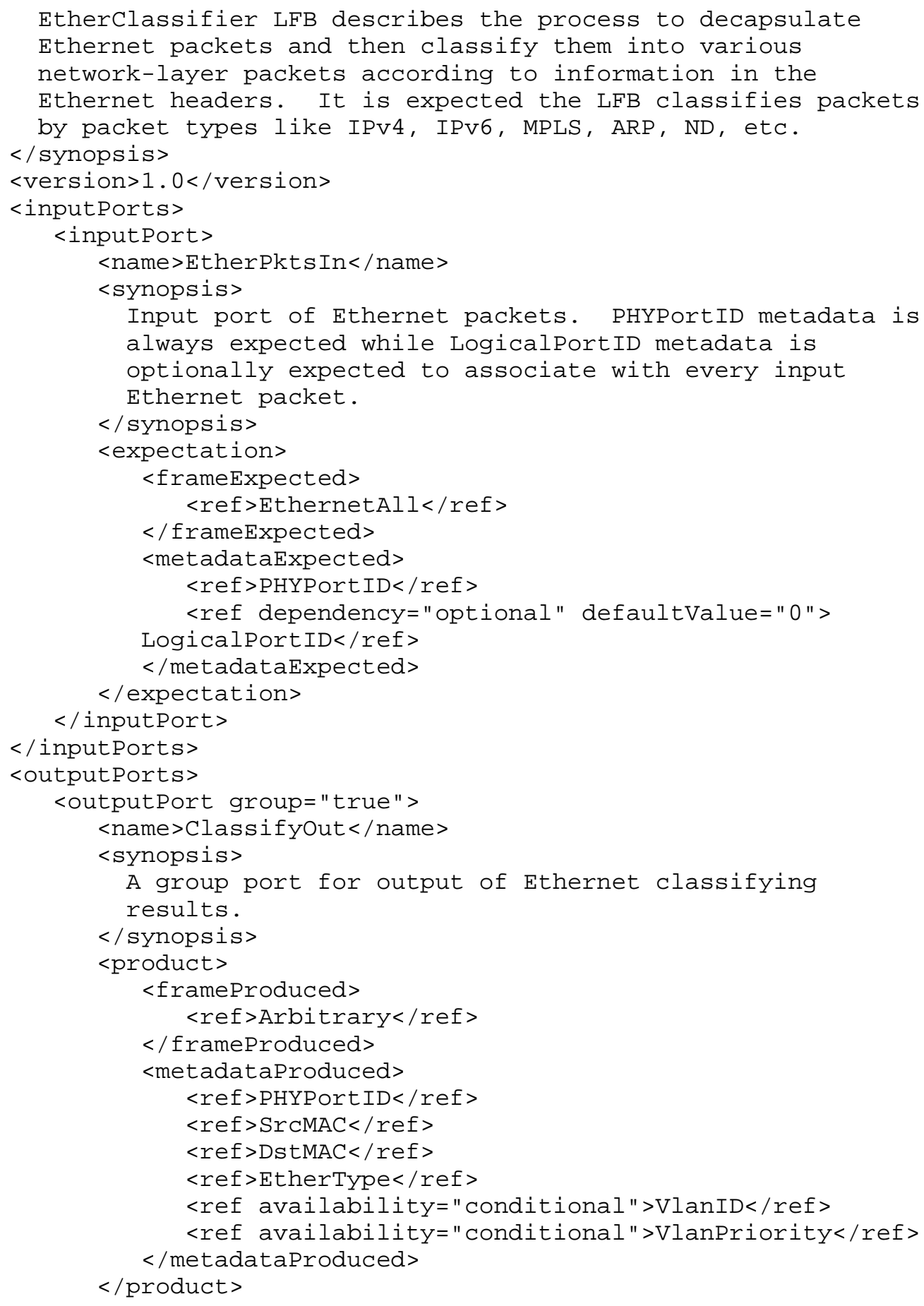

Wang, et al. 


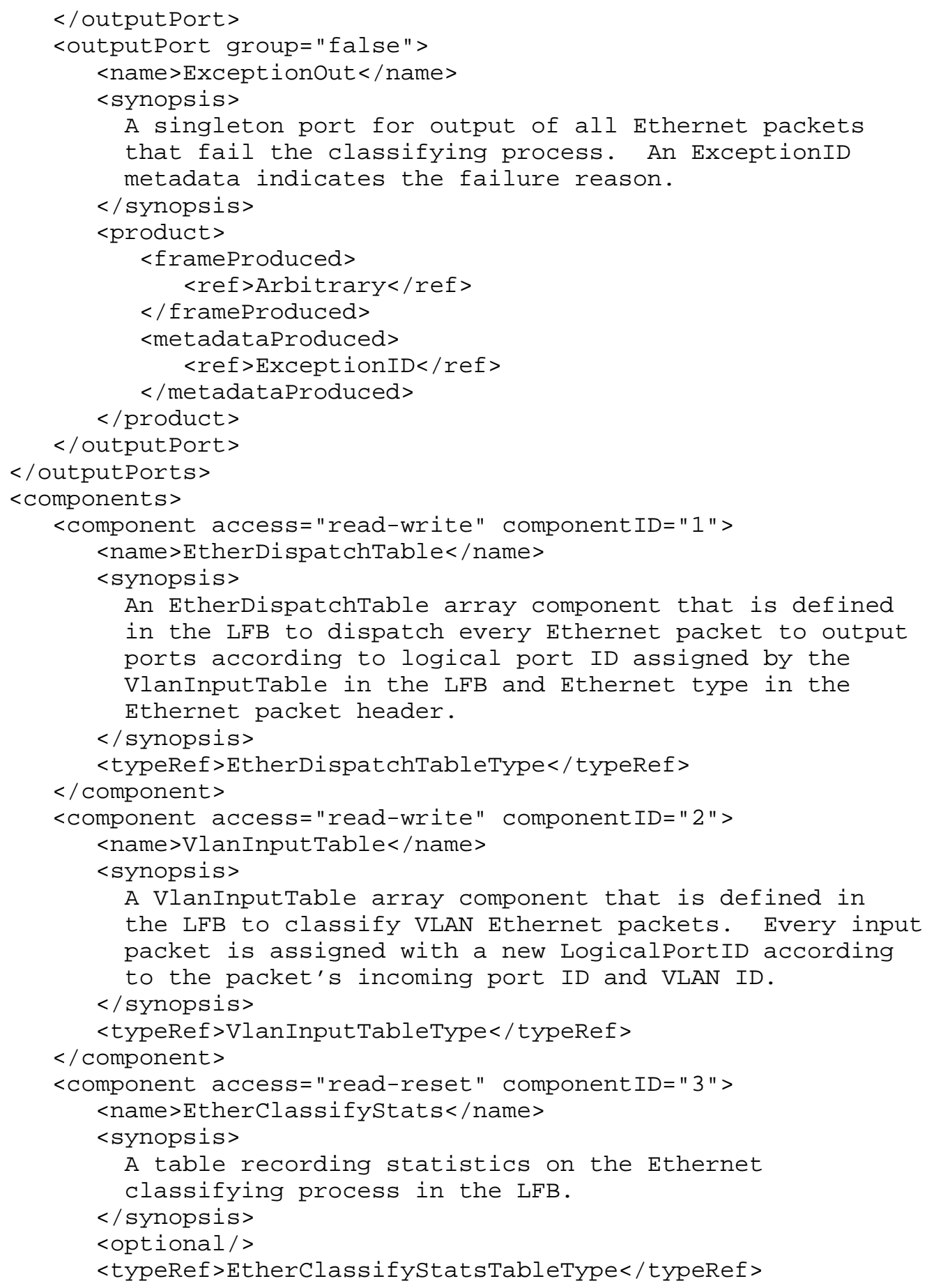

Wang, et al. 


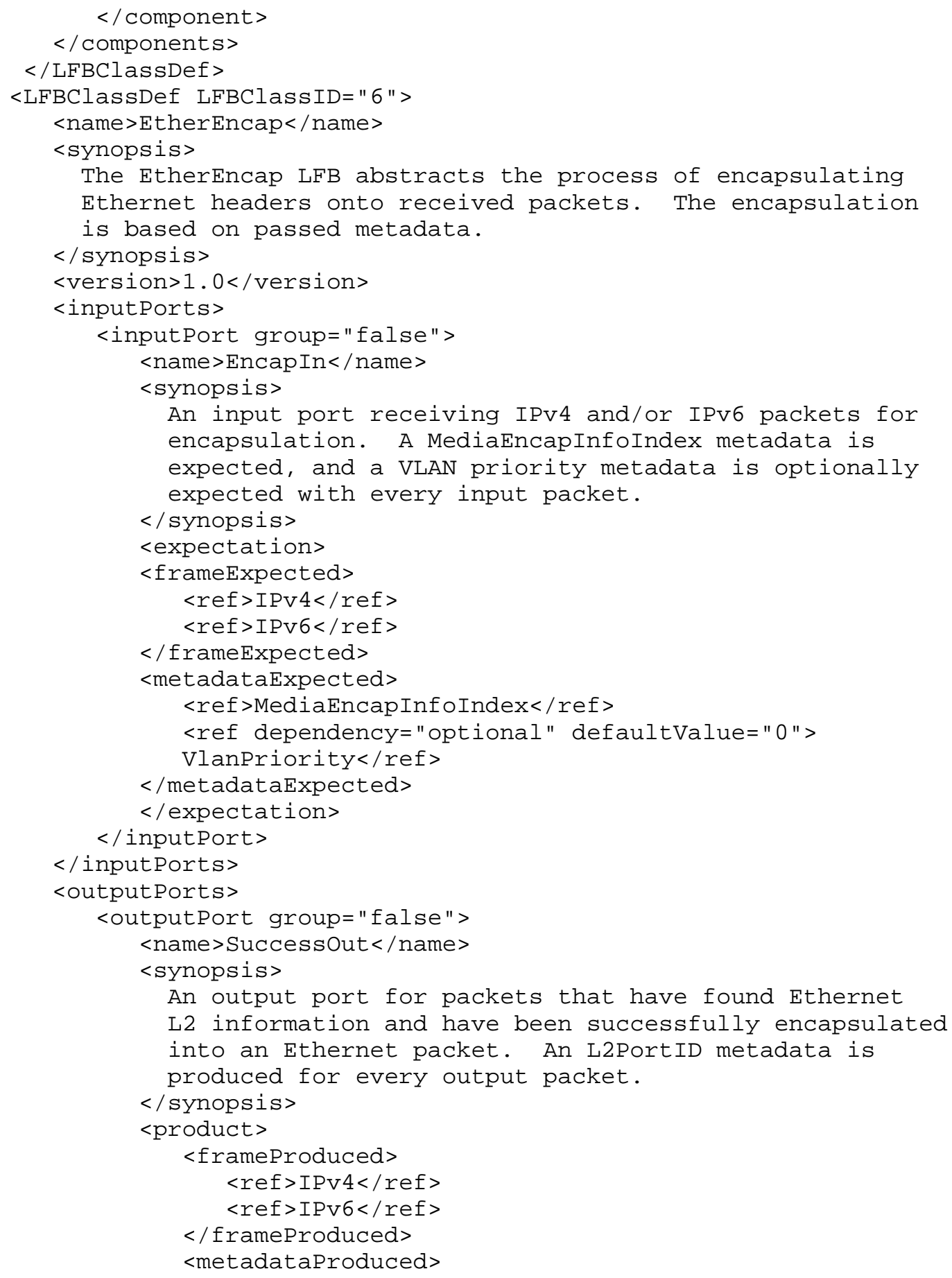




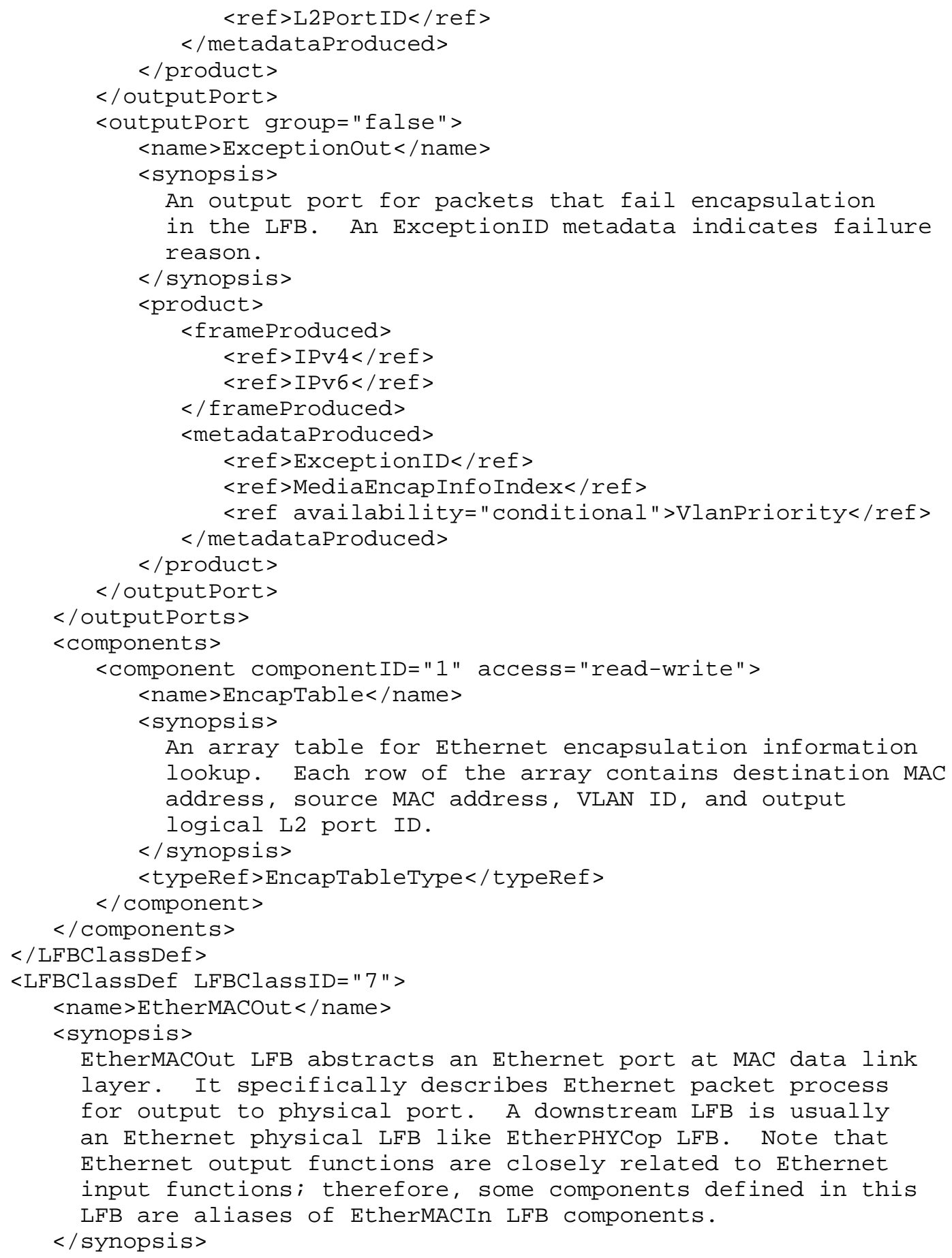




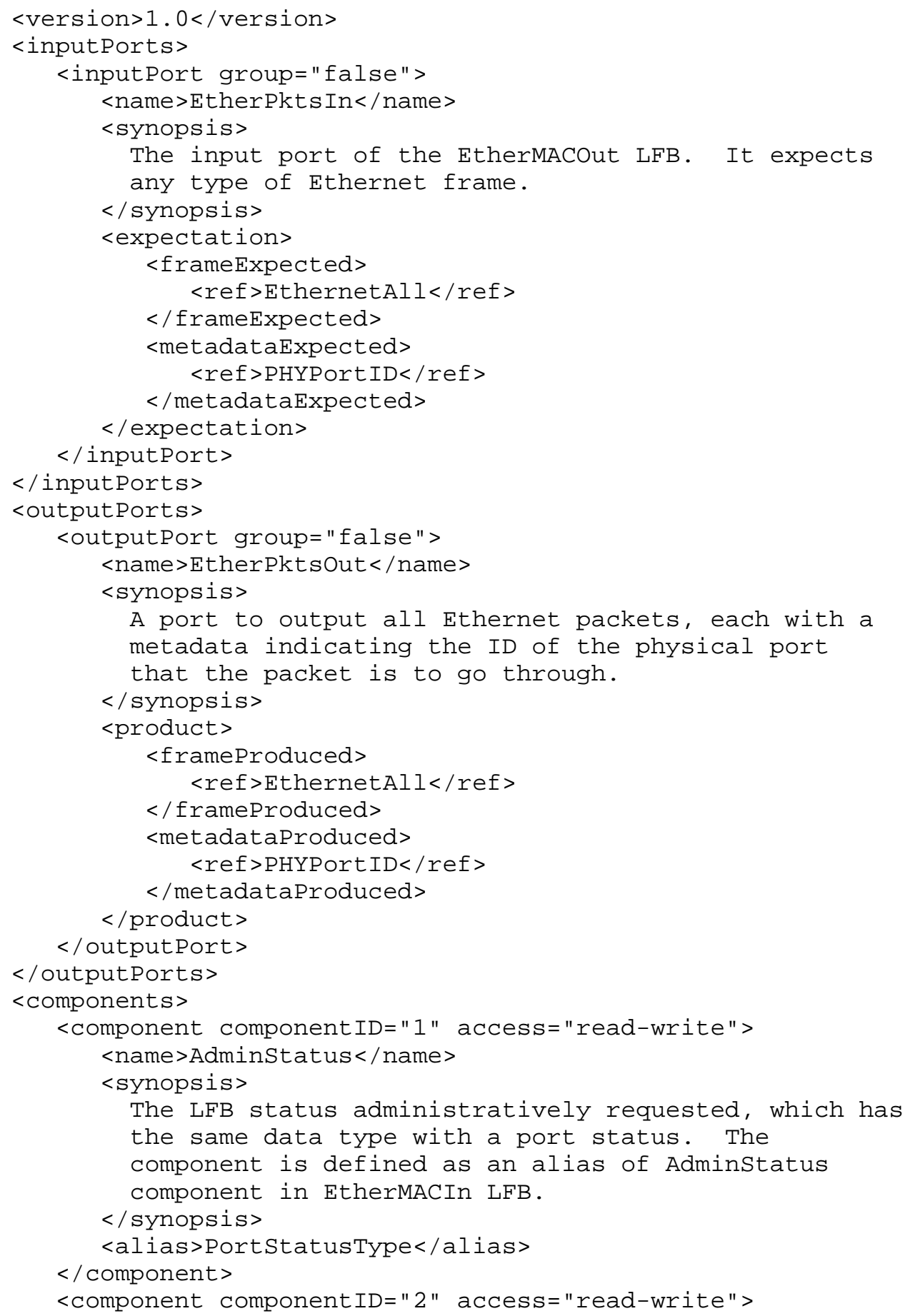




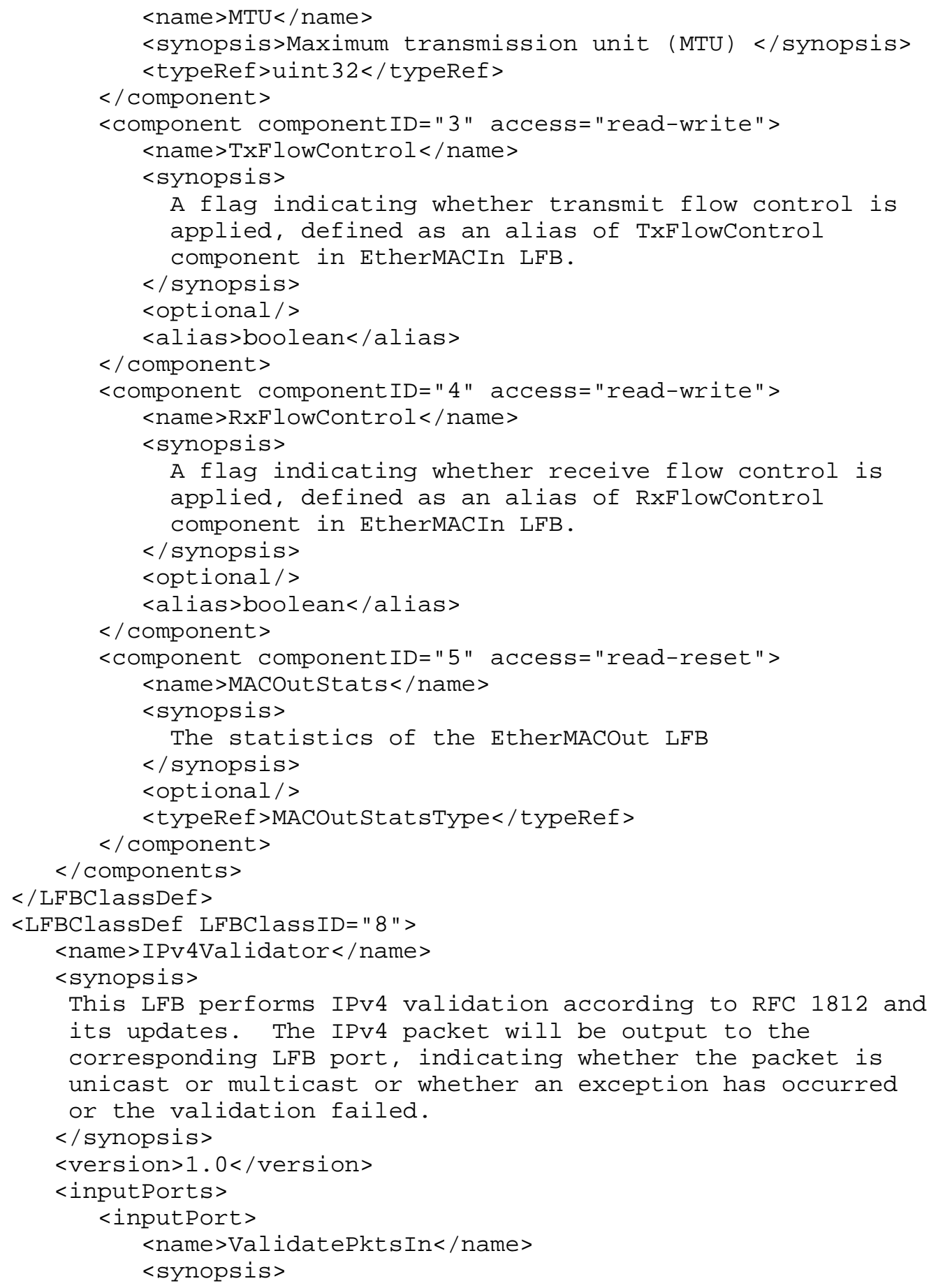




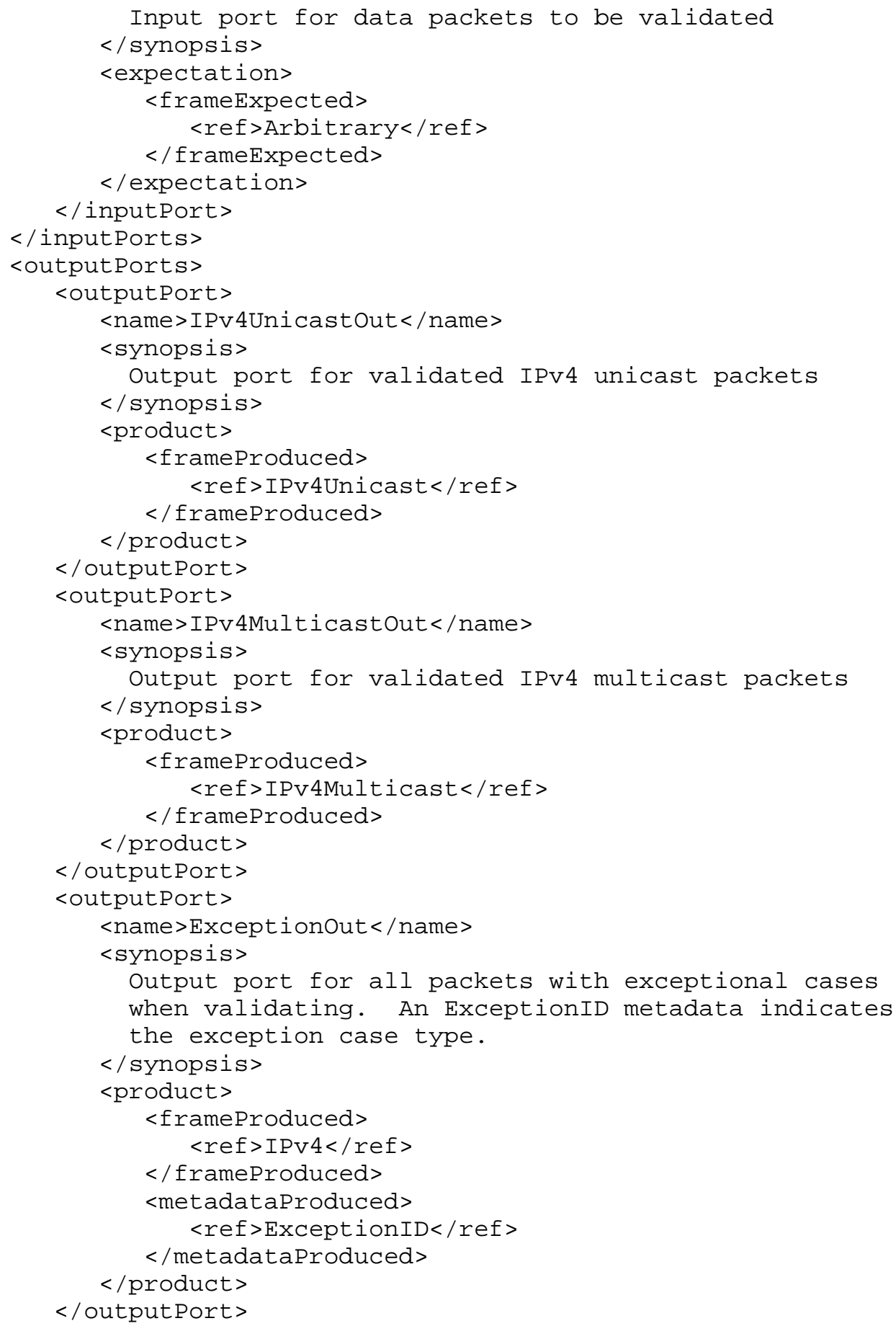

Wang, et al. 


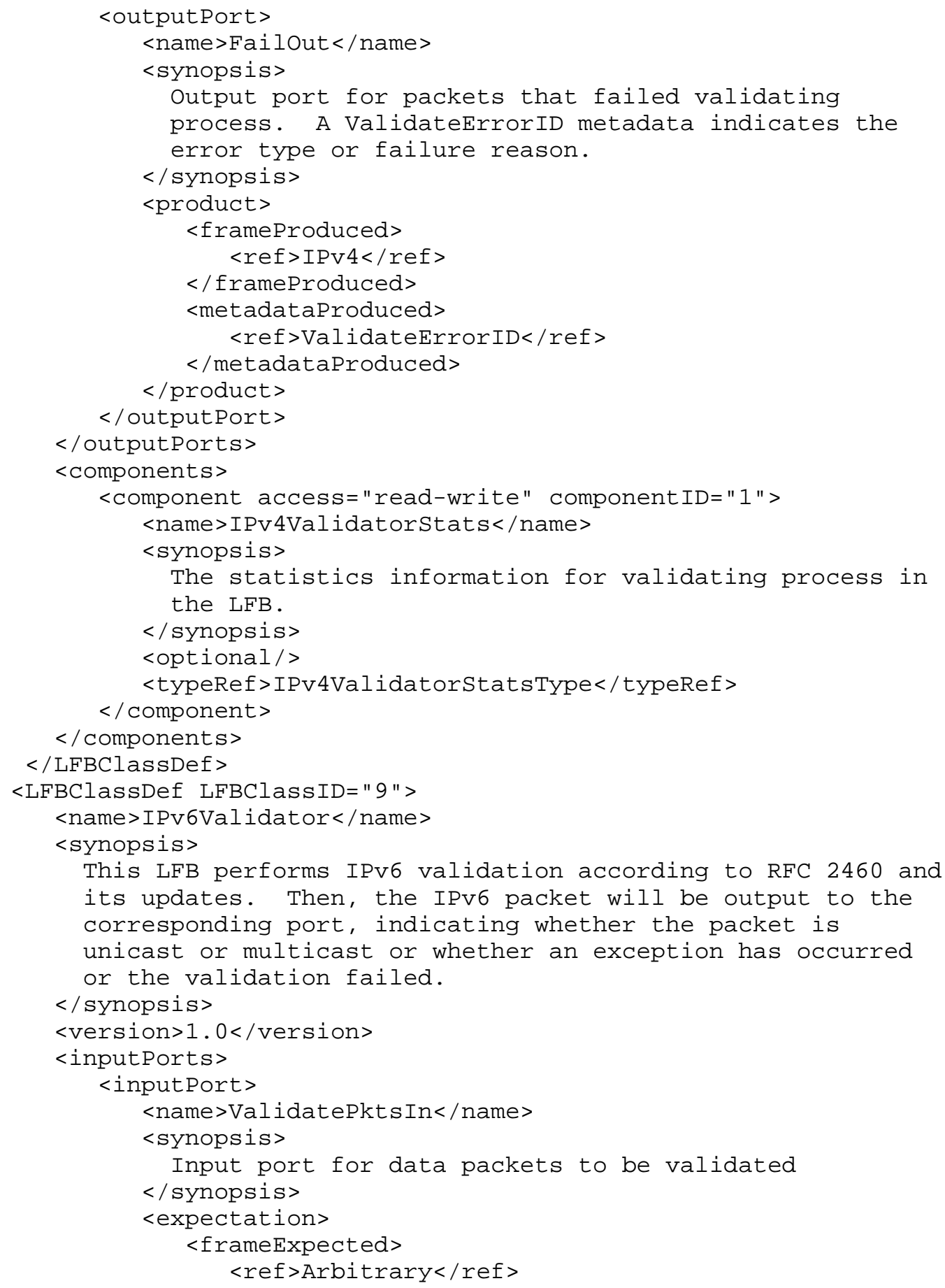




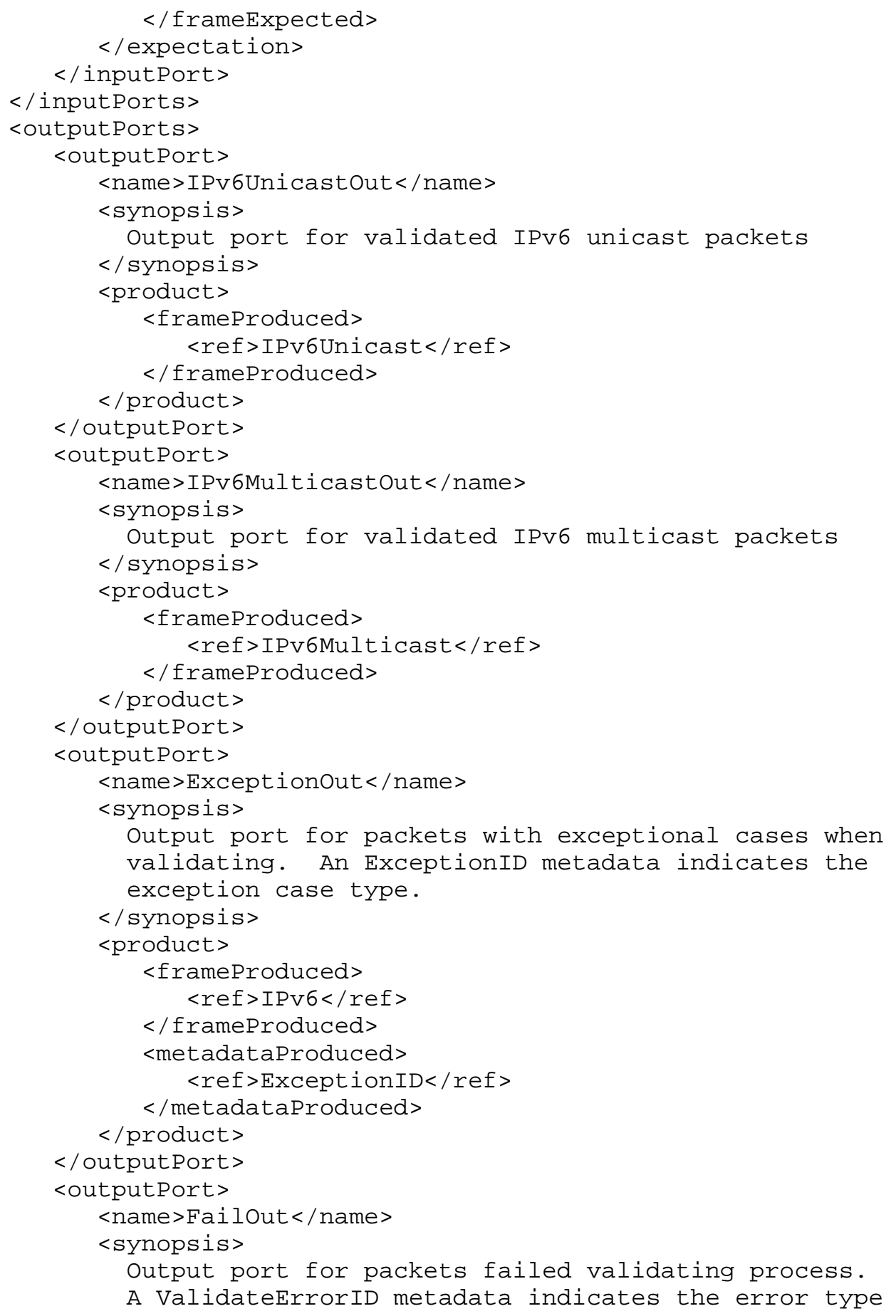




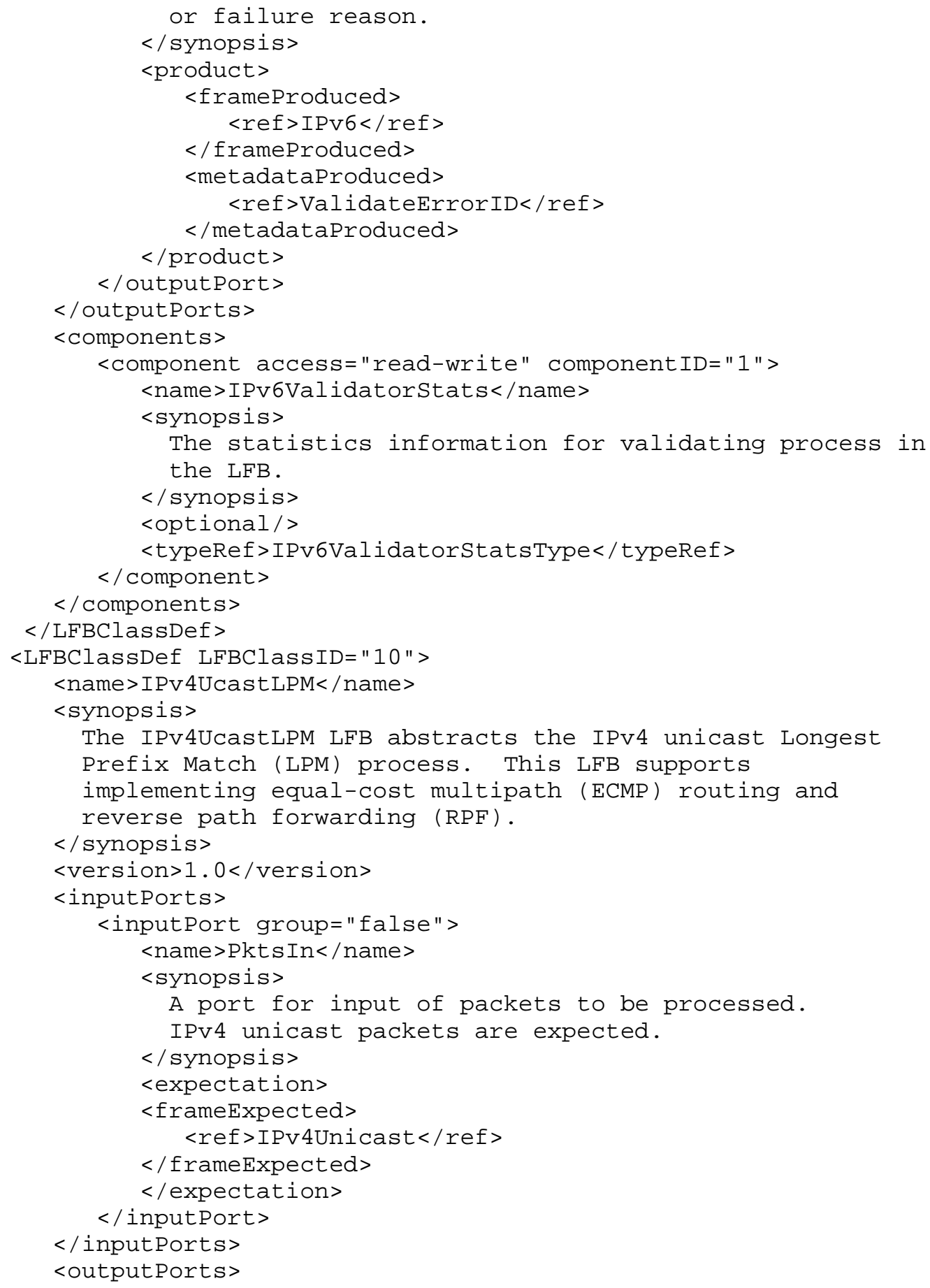




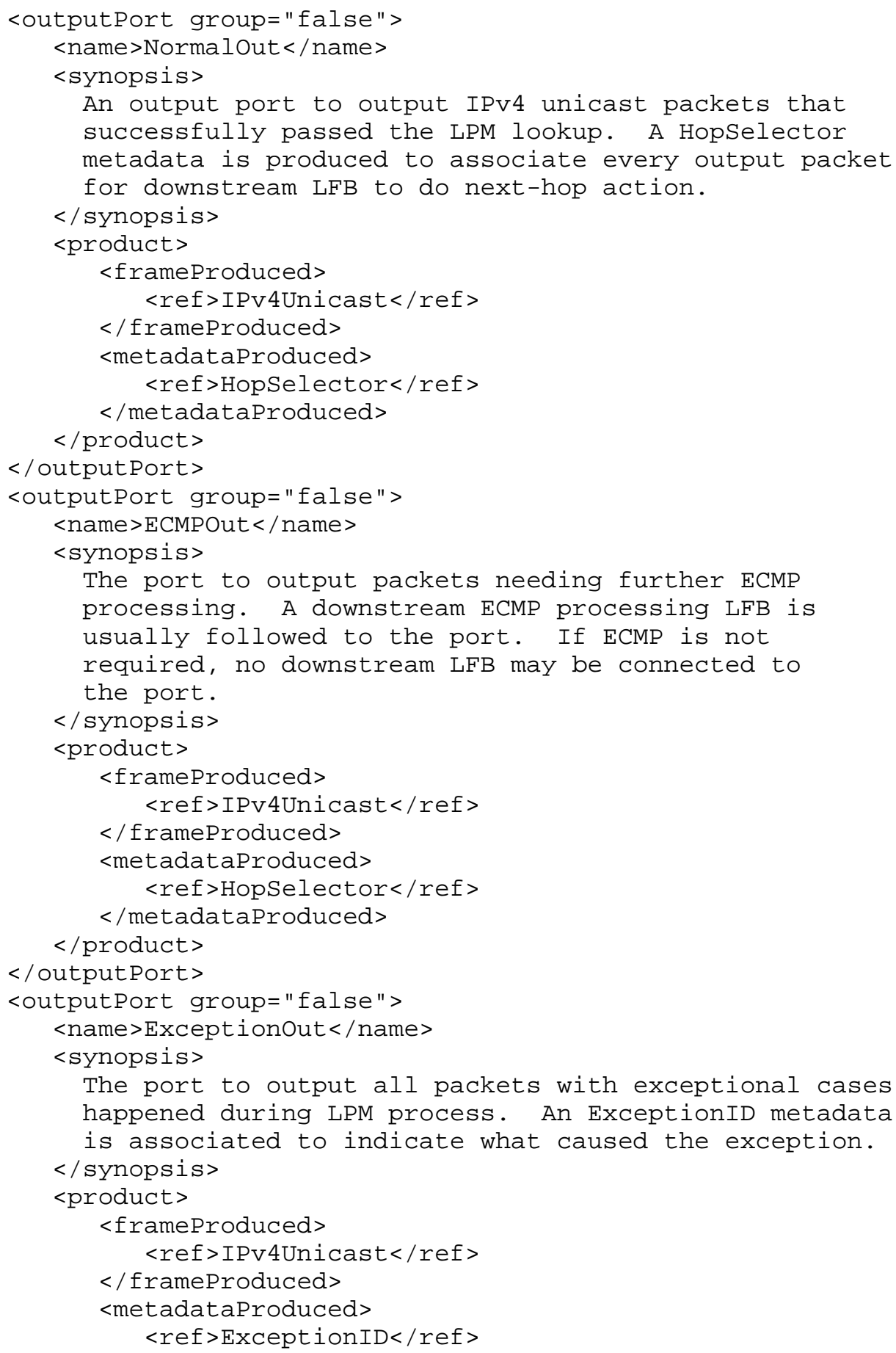

Wang, et al. 


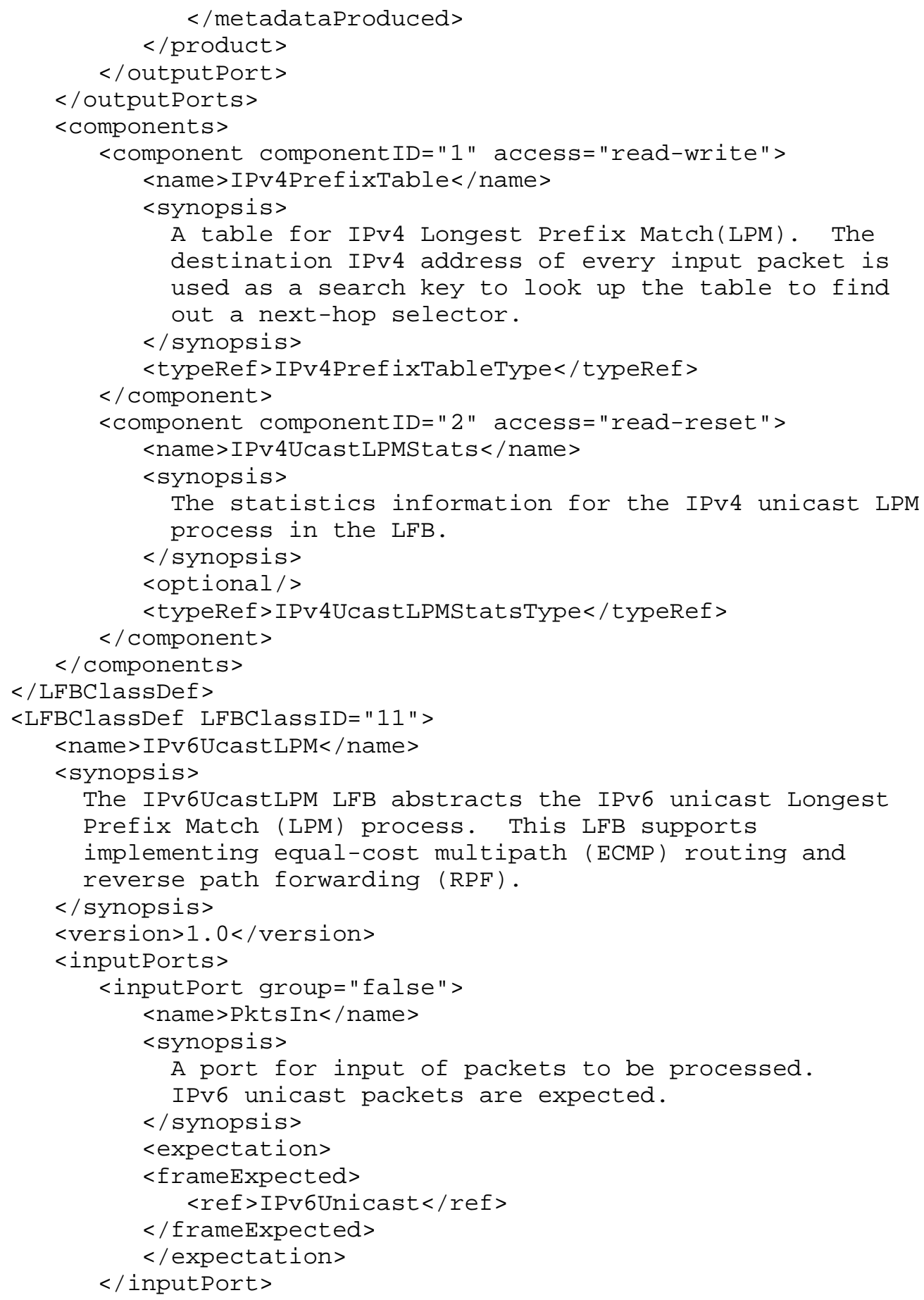




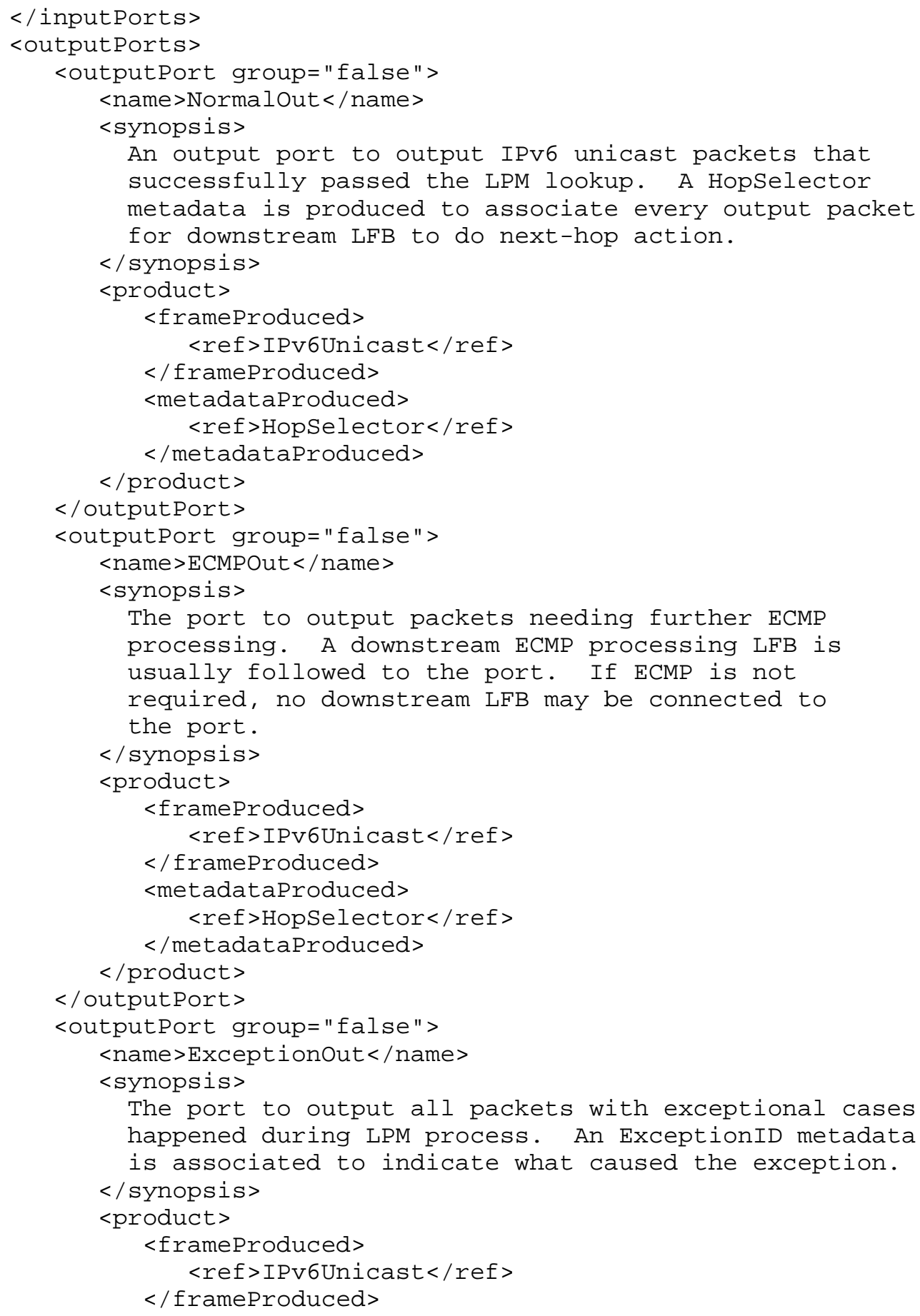

Wang, et al. 


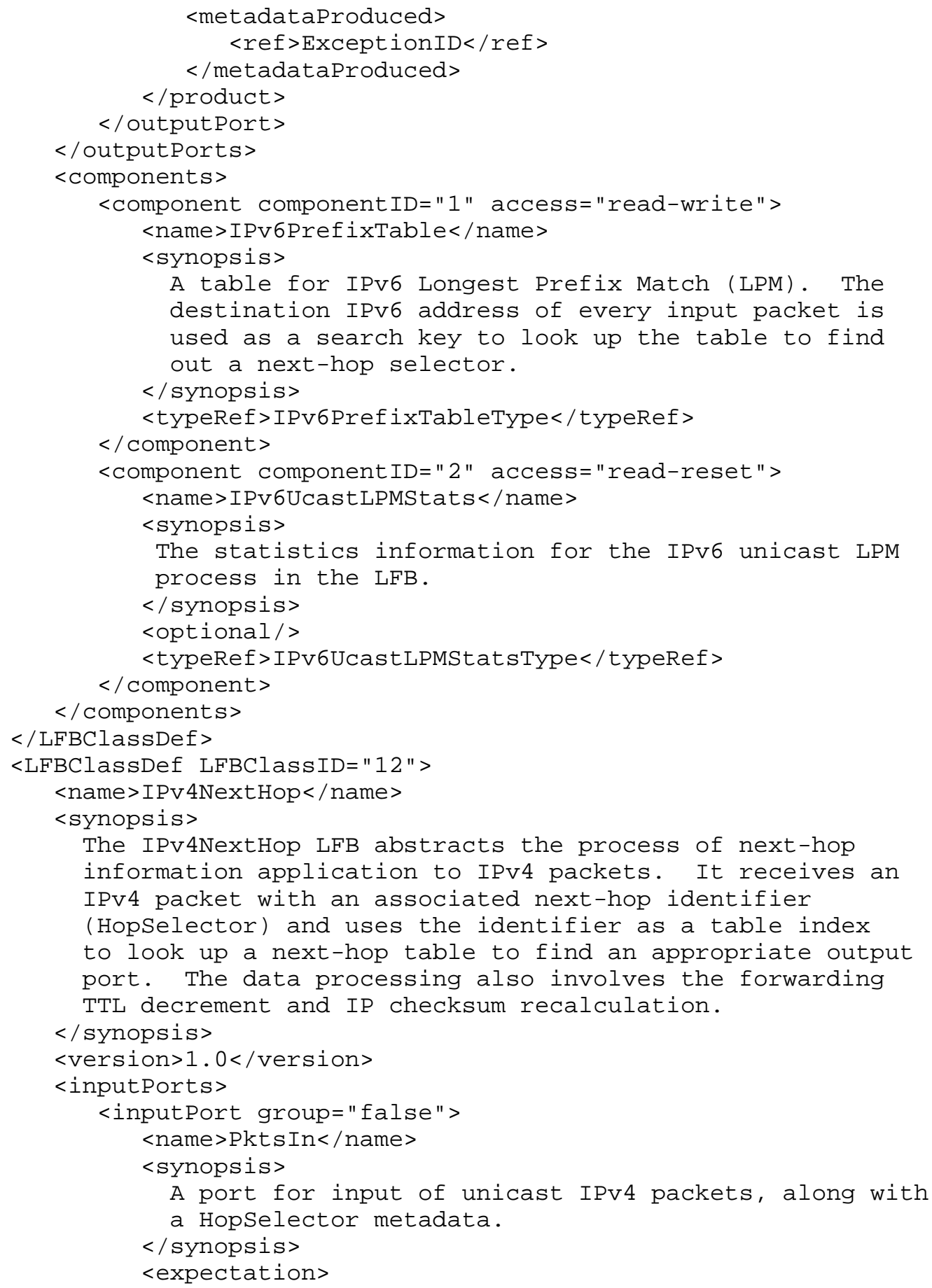




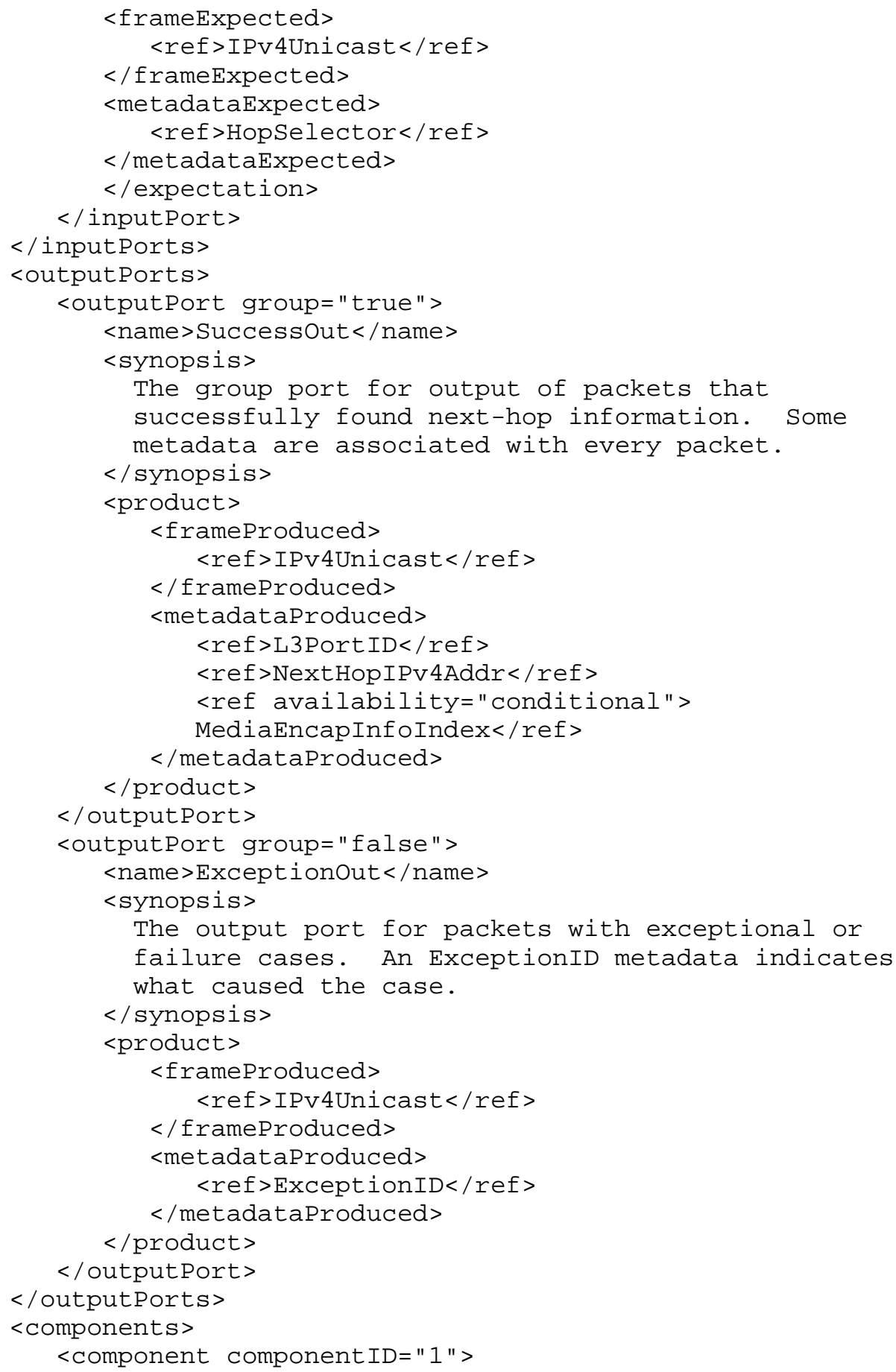




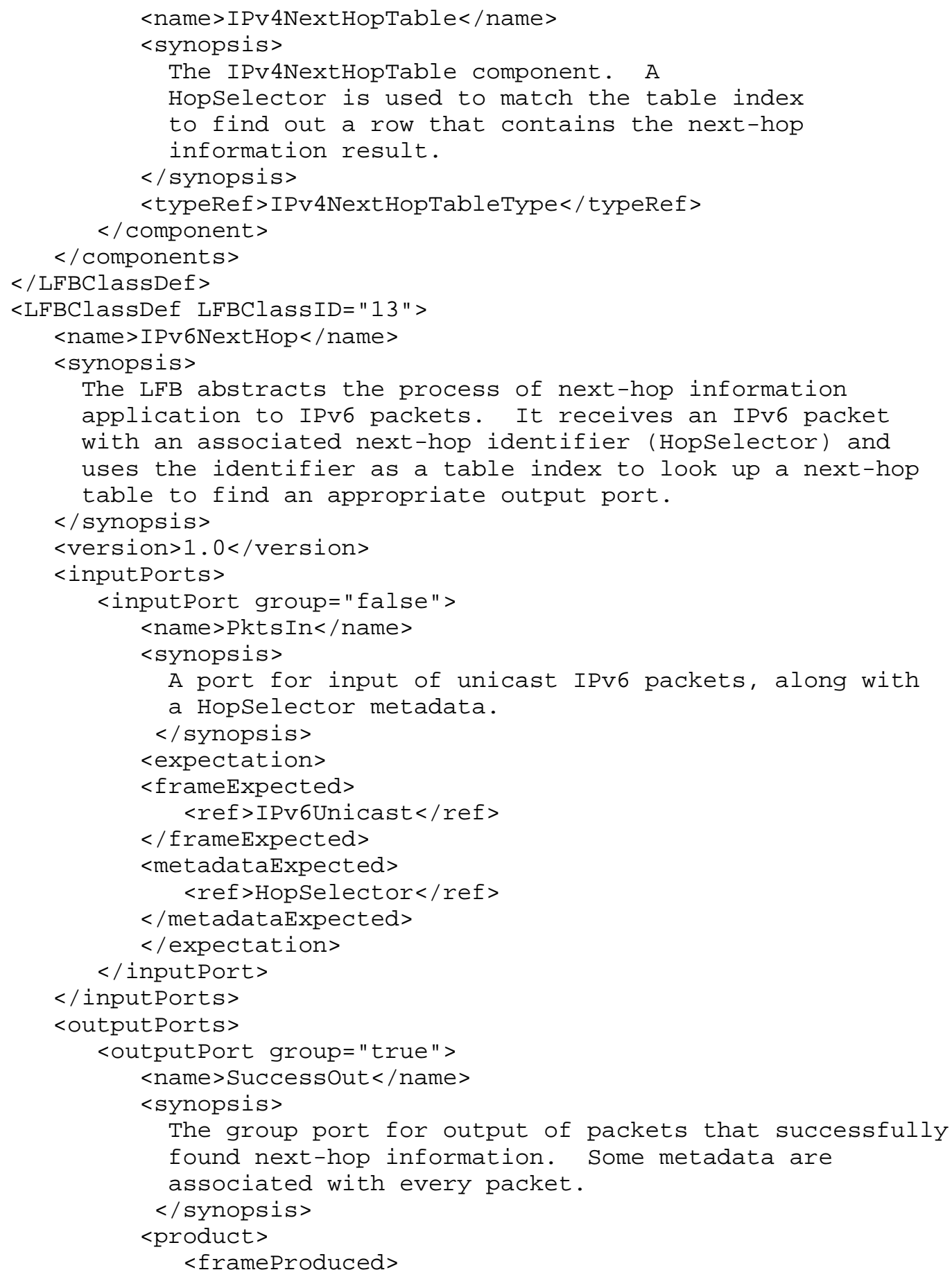




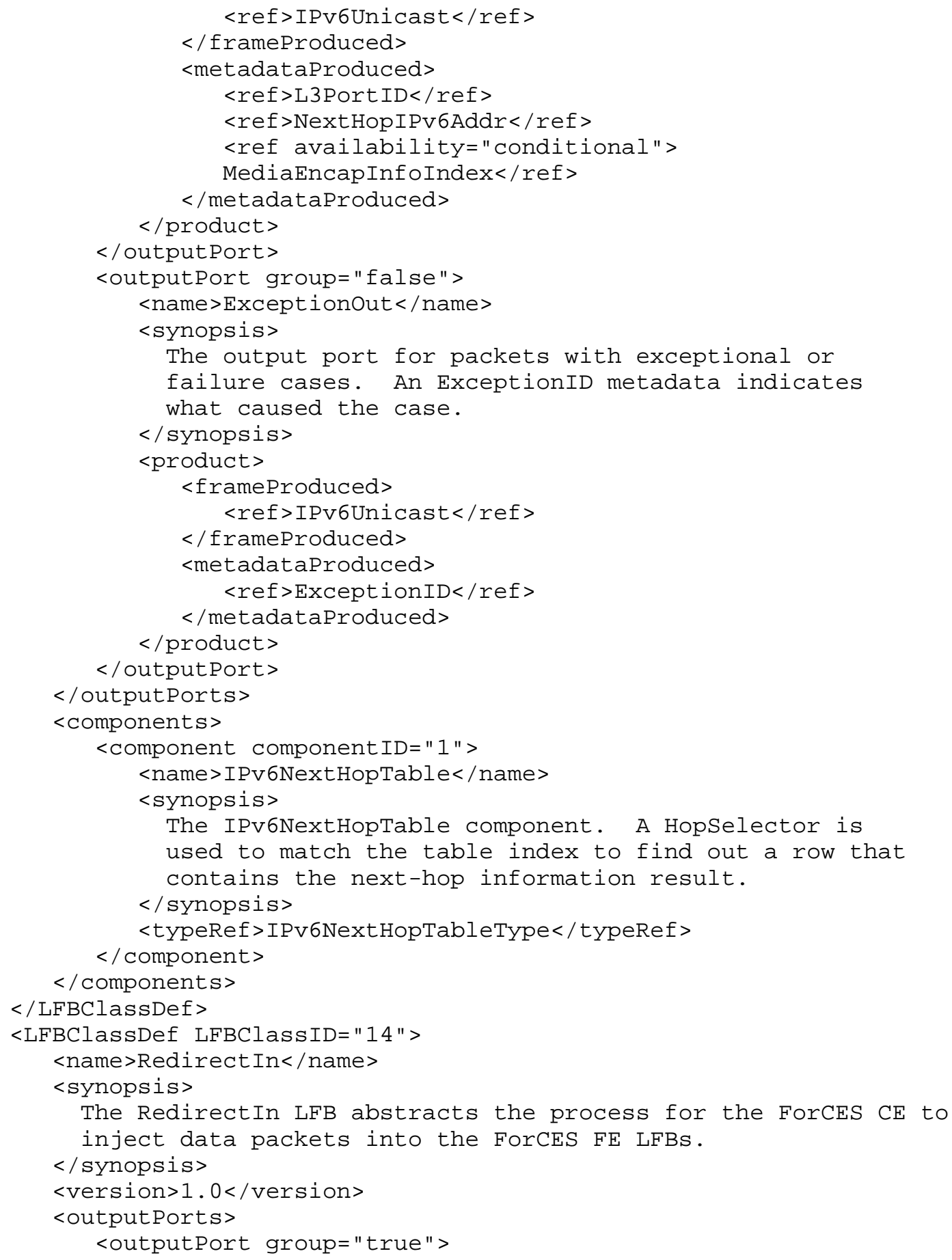




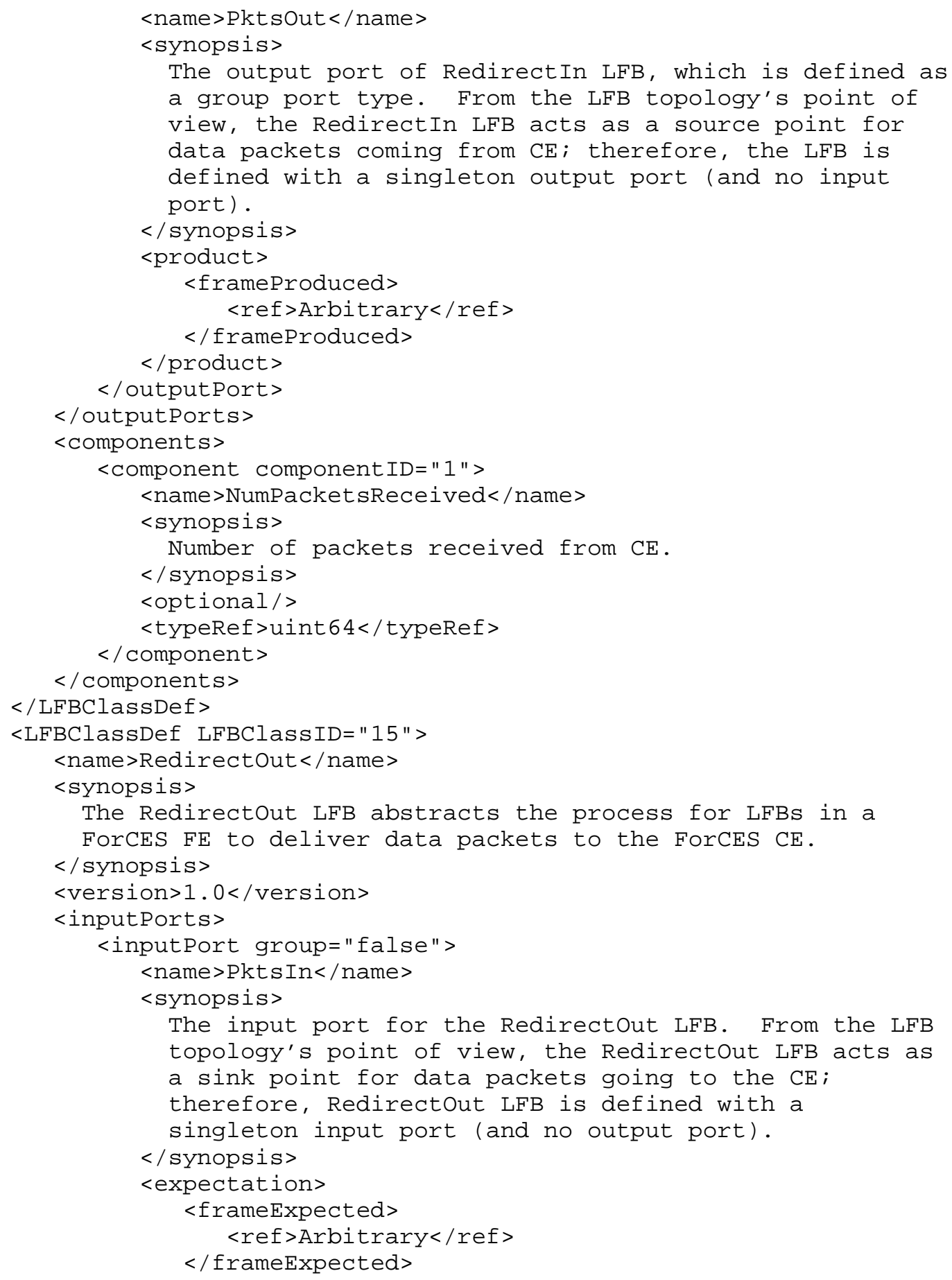

Wang, et al. 


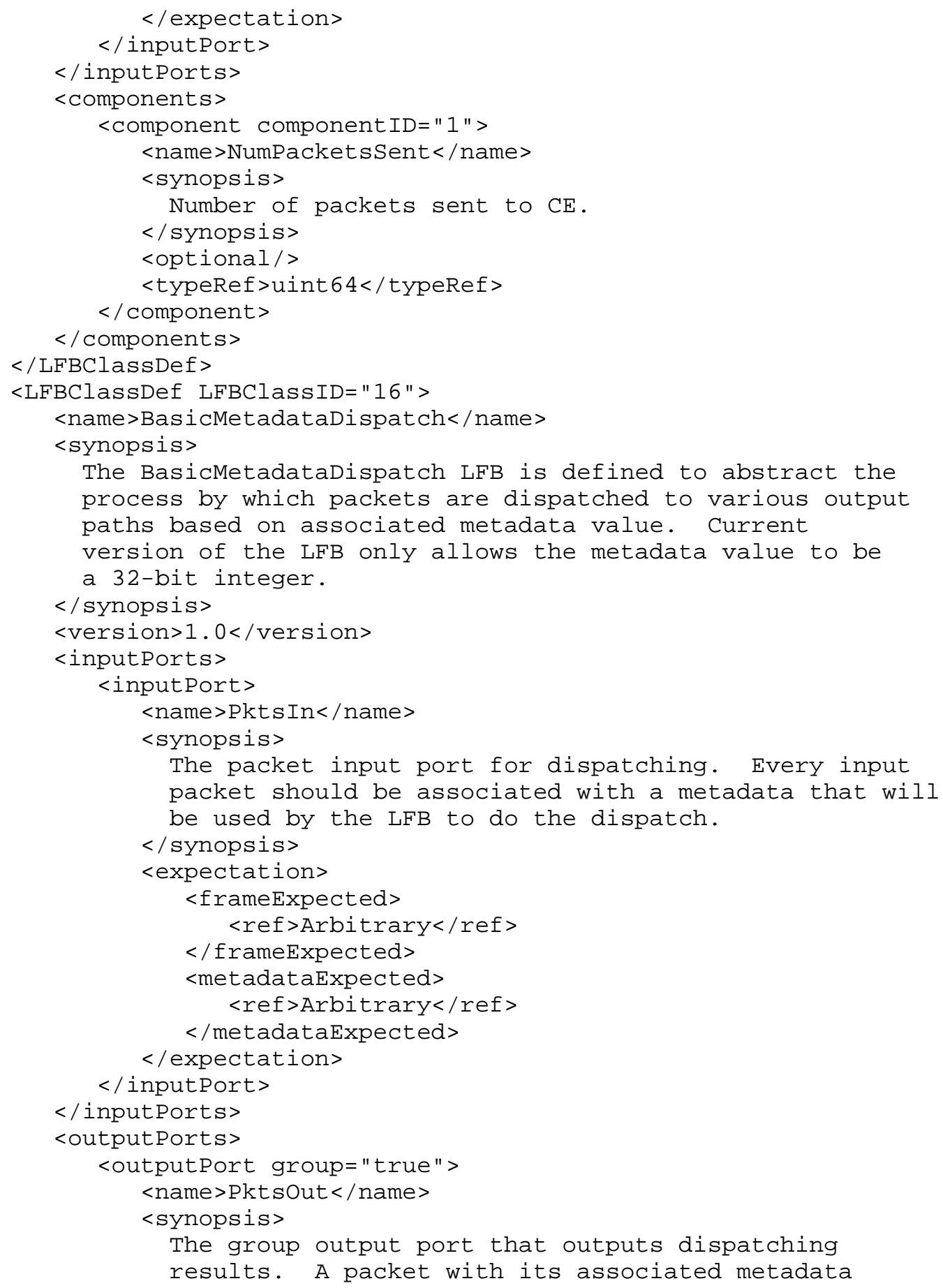




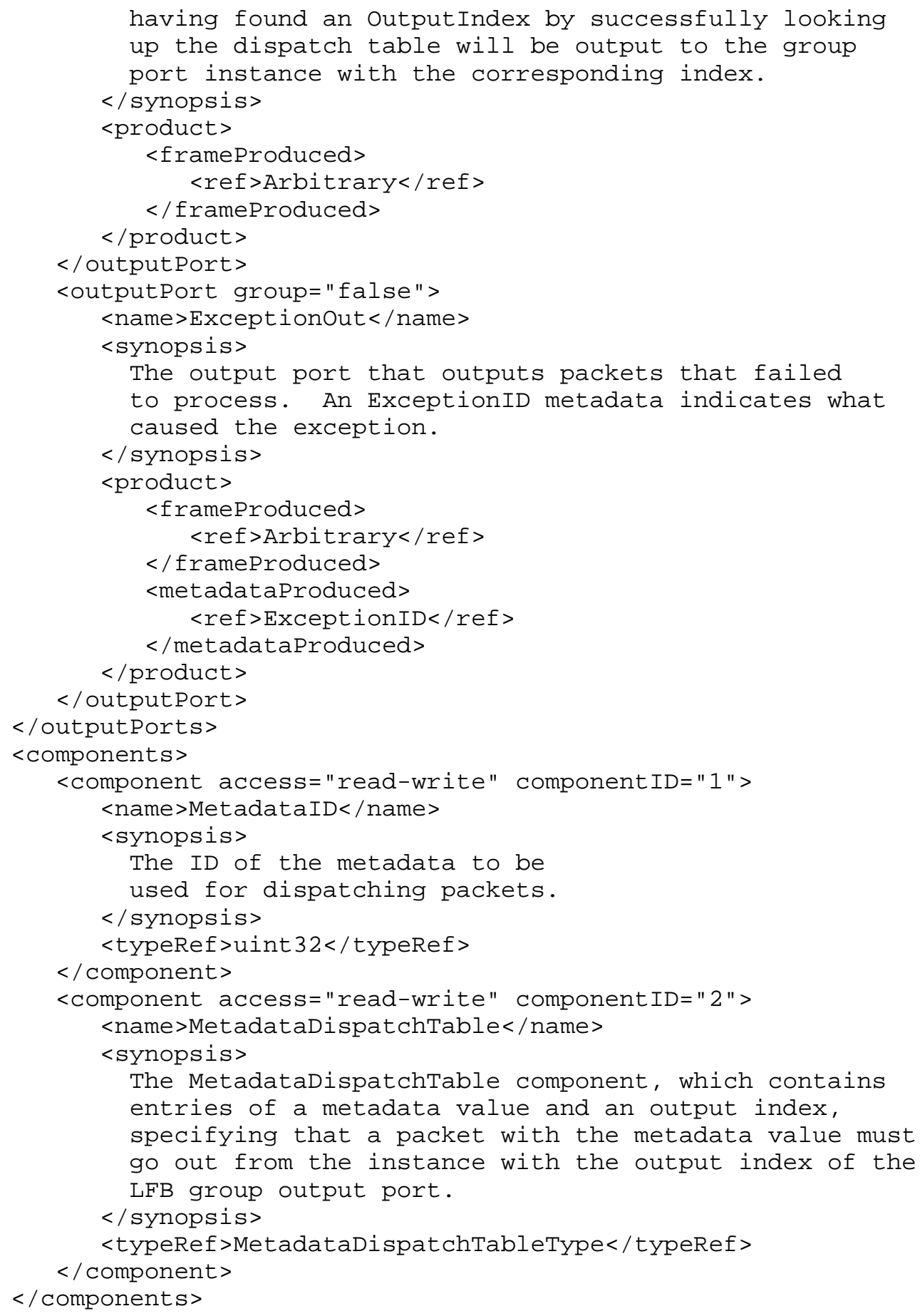




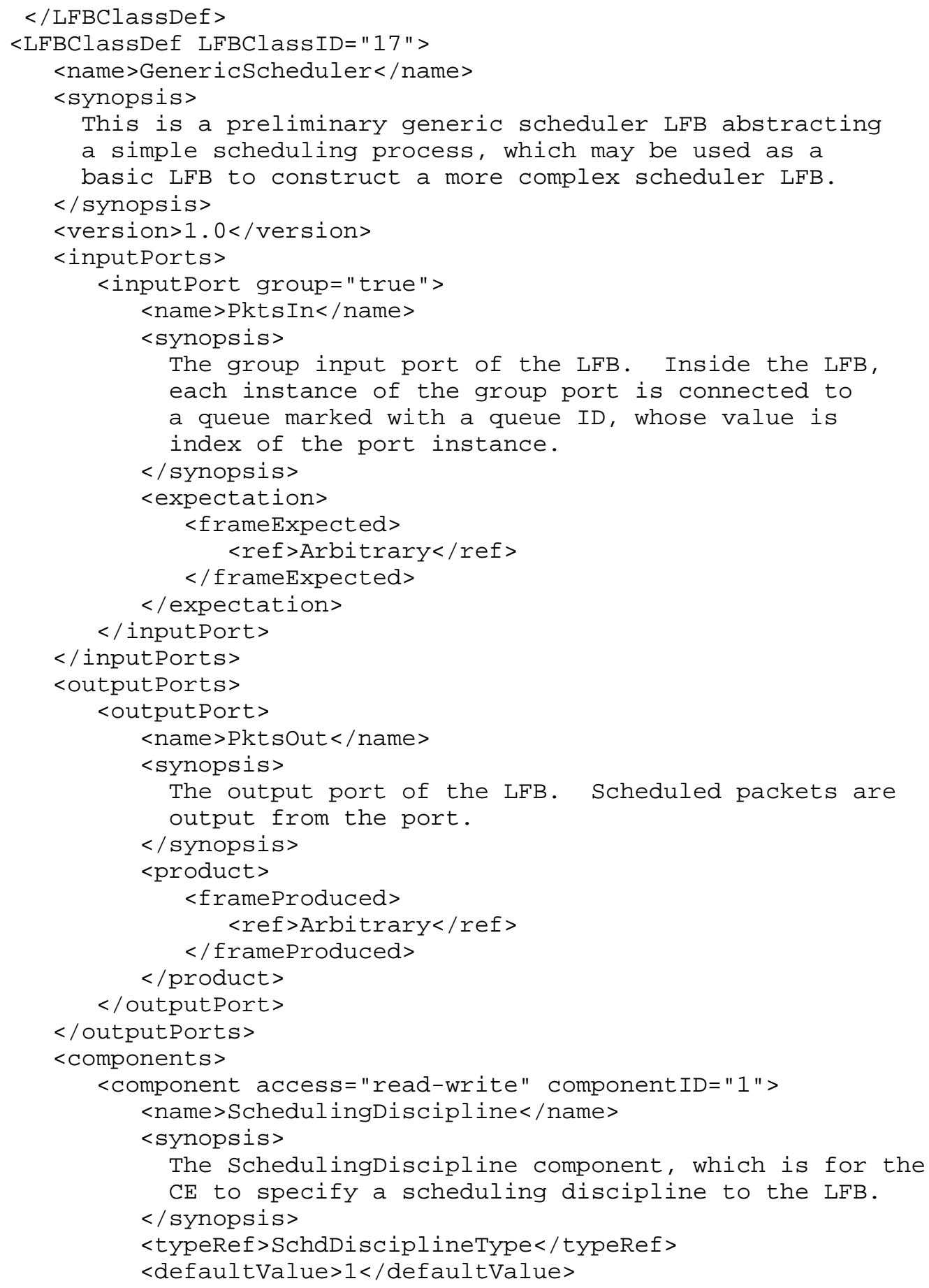

Wang, et al. 


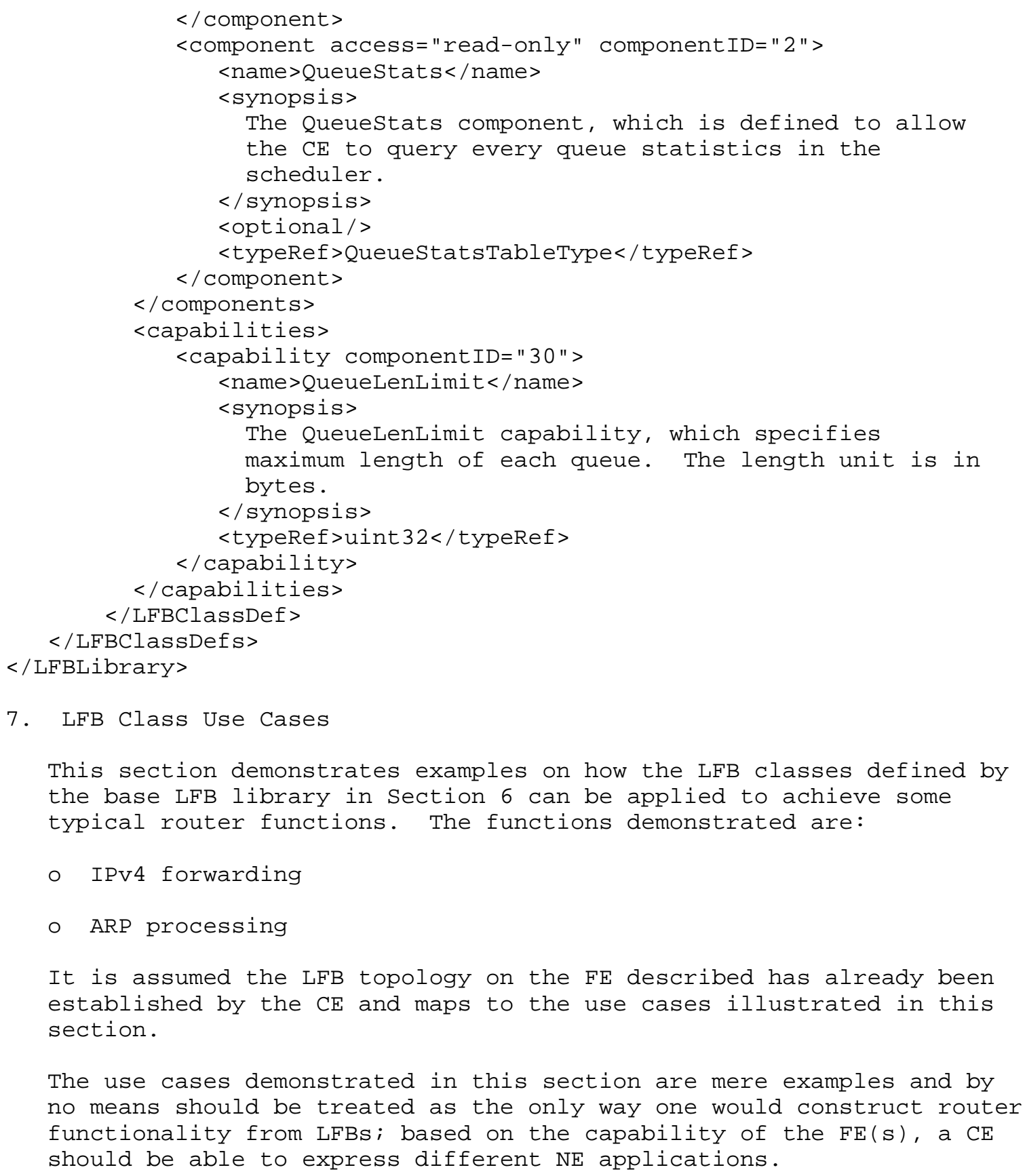

7. LFB Class Use Cases

This section demonstrates examples on how the LFB classes defined by the base LFB library in Section 6 can be applied to achieve some typical router functions. The functions demonstrated are:

- IPv4 forwarding

- ARP processing

It is assumed the LFB topology on the FE described has already been established by the $\mathrm{CE}$ and maps to the use cases illustrated in this section.

The use cases demonstrated in this section are mere examples and by no means should be treated as the only way one would construct router functionality from LFBs; based on the capability of the FE(s), a CE should be able to express different NE applications. 


\subsection{IPv4 Forwarding}

Figure 2 shows the typical LFB processing path for an IPv4 unicast forwarding case with Ethernet media interfaces by use of the base LFB classes. Note that in the figure, to focus on the IP forwarding function, some inputs or outputs of LFBs that are not related to the IPv4 forwarding function are not shown. For example, an EtherClassifier LFB normally has two output ports: a "ClassifyOut" group output port and an "ExceptionOut" singleton output port, with the group port containing various port instances according to various classified packet types (Section 5.1.3). In this figure, only the IPV4 and IPV6 packet output port instances are shown for displaying the mere IPv4 forwarding processing function. 


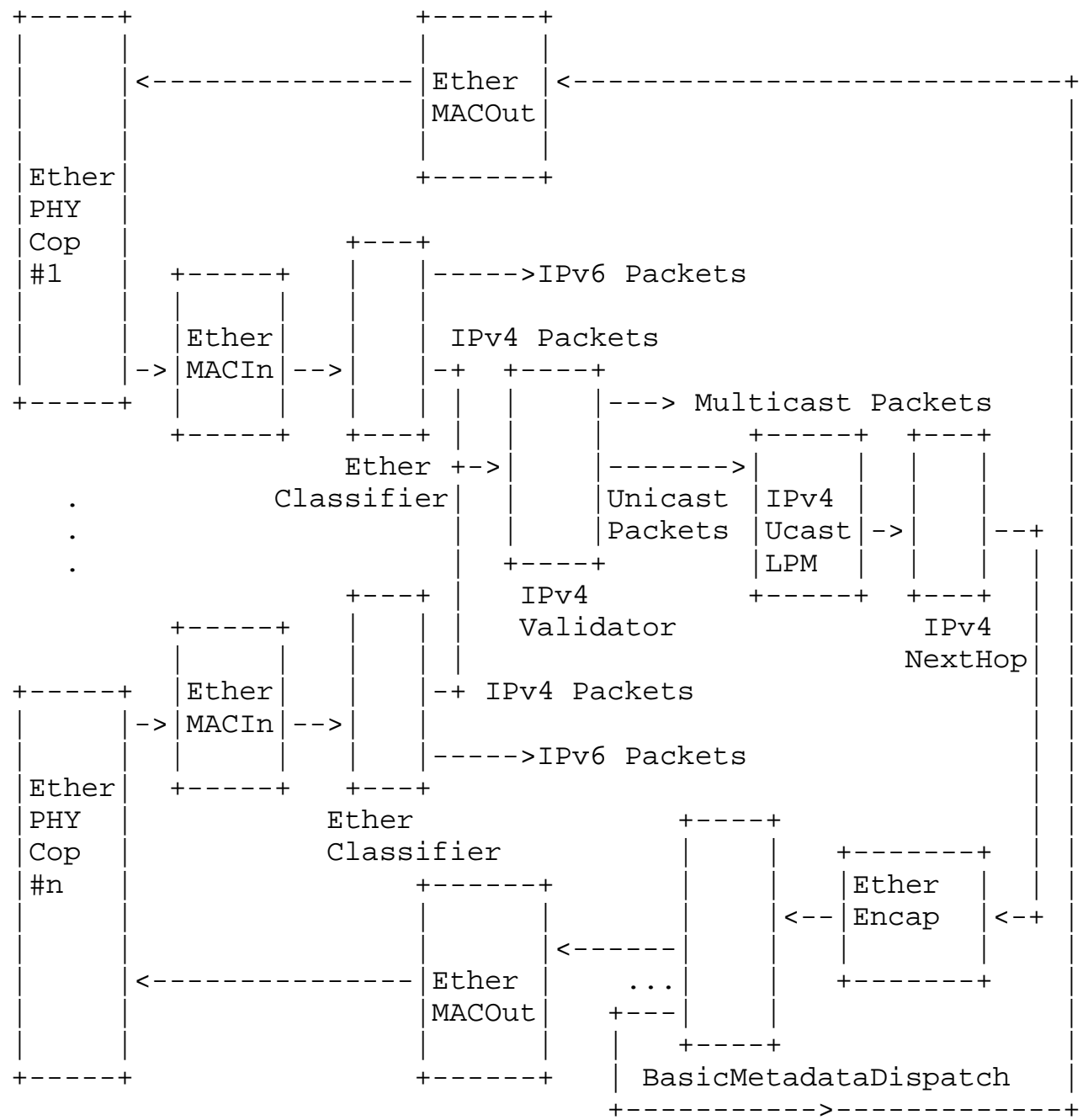

Figure 2: LFB Use Case for IPv4 Forwarding

In the LFB use case, a number of EtherPHYCop LFB (Section 5.1.1) instances are used to describe physical-layer functions of the ports. PHYPortID metadata is generated by the EtherPHYCop LFB and is used by all the subsequent downstream LFBS. An EtherMACIn LFB (Section 5.1.2), which describes the MAC-layer processing, follows every EtherPHYCop LFB. The EtherMACIn LFB may do a locality check of MAC addresses if the CE configures the appropriate EtherMACIn LFB component. 
Ethernet packets out of the EtherMACIn LFB are sent to an EtherClassifier LFB (Section 5.1.3) to be decapsulated and classified into network-layer types like IPV4, IPv6, ARP, etc. In the example use case, every physical Ethernet interface is associated with one Classifier instance; although not illustrated, it is also feasible that all physical interfaces are associated with only one Ethernet Classifier instance.

EtherClassifier uses the PHYPortID metadata, the Ethernet type of the input packet, and VlanID (if present in the input Ethernet packets) to decide the packet network-layer type and the LFB output port to the downstream LFB. The EtherClassifier LFB also assigns a new logical port ID metadata to the packet for later use. The Etherclassifier may also generate some new metadata for every packet, like EtherType, SrCMAC, DstMAC, LogicPortID, etc., for consumption by downstream LFBs.

If a packet is classified as an IPv4 packet, it is sent downstream to an IPv4Validator LFB (Section 5.2.1) to validate the IPv4 packet. In the validator LFB, IPV4 packets are validated and are additionally classified into either IPv4 unicast packets or multicast packets. IPv4 unicast packets are sent to downstream to the IPv4UcastLPM LFB (Section 5.3.1).

The IPV4UcastLPM LFB is where the longest prefix match decision is made, and a next-hop selection is selected. The next-hop ID metadata is generated by the IPV4UcastLPM LFB to be consumed downstream by the IPv4NextHop LFB (Section 5.3.2).

The IPv4NextHop LFB uses the next-hop ID metadata to derive where the packet is to go next and the media encapsulation type for the port, etc. The IPv4NextHop LFB generates the L3PortID metadata used to identify a next-hop output physical/logical port. In the example use case, the next-hop output port is an Ethernet type; as a result, the packet and its L3 port ID metadata are sent downstream to an EtherEncap LFB (Section 5.1.4).

The EtherEncap LFB encapsulates the incoming packet into an Ethernet frame. A BasicMetadataDispatch LFB (Section 5.5.1) follows the EtherEncap LFB. The BasicMetadataDispatch LFB is where packets are finally dispatched to different output physical/logical ports based on the L3Portid metadata sent to the LFB. 


\subsection{ARP Processing}

Figure 3 shows the processing path for the Address Resolution Protocol (ARP) in the case the CE implements the ARP processing function. By no means is this the only way ARP processing could be achieved; as an example, ARP processing could happen at the FE, but that discussion is out of the scope of this use case.

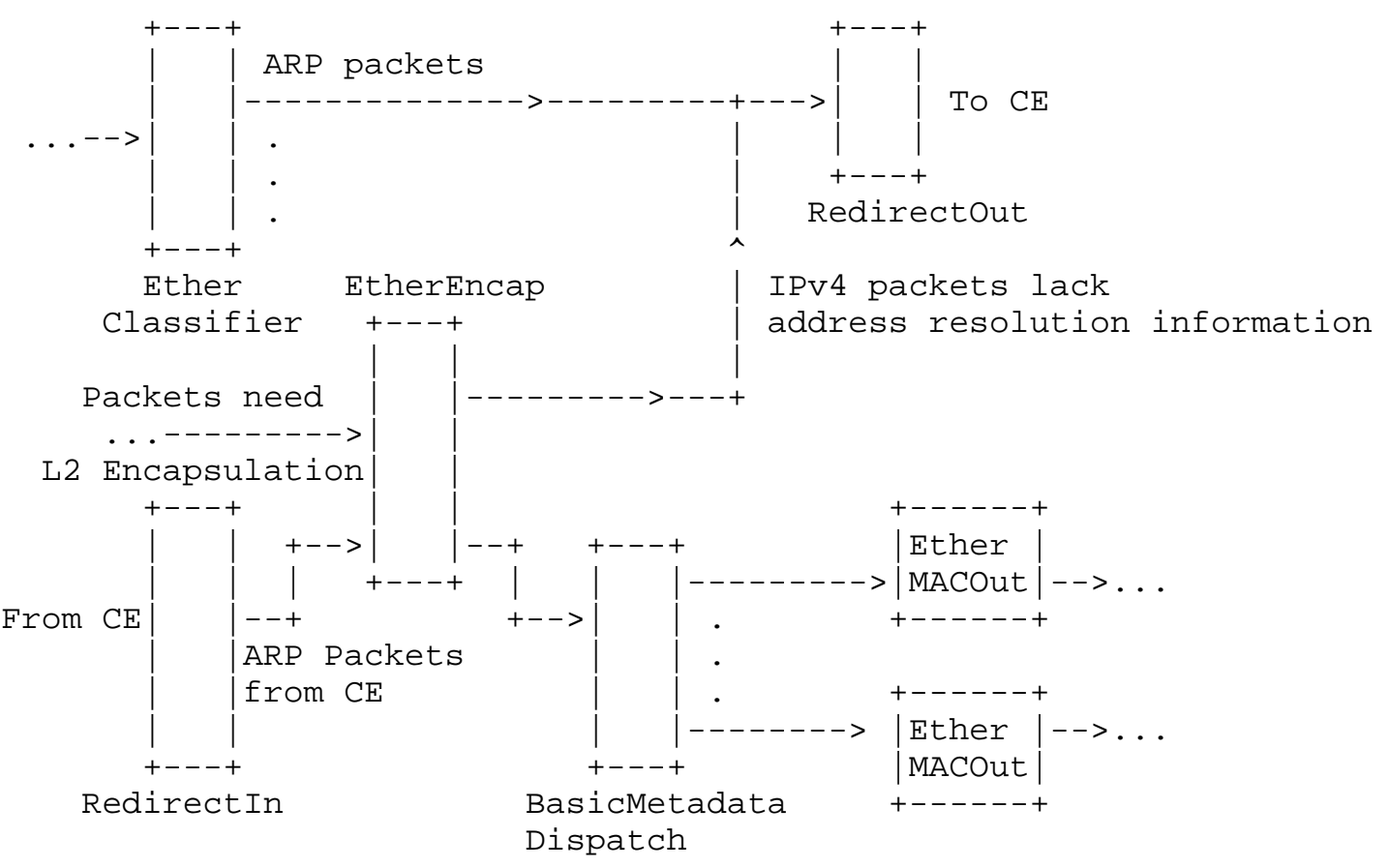

Figure 3: LFB Use Case for ARP

There are two ways ARP processing could be triggered in the CE as illustrated in Figure 3:

- ARP packets arriving from outside of the NE.

- IPV4 packets failing to resolve within the FE.

ARP packets from network interfaces are filtered out by EtherClassifier LFB. The classified ARP packets and associated metadata are then sent downstream to the Redirectout LFB (Section 5.4.2) to be transported to $\mathrm{CE}$. 
The EtherEncap LFB, as described in Section 5.1.4, receives packets that need Ethernet L2 encapsulating. When the EtherEncap LFB fails to find the necessary L2 Ethernet information with which to encapsulate the packet, it outputs the packet to its Exceptionout LFB port. Downstream to EtherEncap LFB's Exceptionout LFB port is the RedirectOut LFB, which transports the packet to the CE (see Section 5.1.4 on EtherEncap LFB for details).

To achieve its goal, the $\mathrm{CE}$ needs to generate $\mathrm{ARP}$ request and response packets and send them to external (to the NE) networks. ARP request and response packets from the $\mathrm{CE}$ are redirected to an $\mathrm{FE}$ via a RedirectIn LFB (Section 5.4.1).

As was the case with forwarded IPv4 packets, outgoing ARP packets are also encapsulated to Ethernet format by the EtherEncap LFB, and then dispatched to different interfaces via a BasicMetadataDispatch LFB. The BasicMetadataDispatch LFB dispatches the packets according to the L3PortID metadata included in every ARP packet sent from CE.

8. IANA Considerations

IANA has created a registry of ForCES LFB class names and the corresponding ForCES LFB Class identifiers, with the location of the definition of the ForCES LFB class, in accordance with the rules to use the namespace.

This document registers the unique class names and numeric class identifiers for the LFBs listed in section 8.1. Besides, this document defines the following namespaces:

- Metadata ID, defined in Sections 4.3 and 4.4

- Exception ID, defined in Section 4.4

- Validate Error ID, defined in Section 4.4 


\subsection{LFB Class Names and LFB Class Identifiers}

LFB classes defined by this document belong to LFBs defined by Standards Track RFCs. According to IANA, the registration procedure is Standards Action for the range 0 to 65535 and First Come First Served with any publicly available specification for over 65535 .

The assignment of LFB class names and LFB class identifiers is as in the following table.

\begin{tabular}{|c|c|c|c|}
\hline $\begin{array}{l}\text { LFB Class } \\
\text { Identifier }\end{array}$ & LFB Class Name & Description & Reference \\
\hline 3 & EtherPHYCop & $\begin{array}{l}\text { Define an Ethernet port } \\
\text { abstracted at physical } \\
\text { layer. }\end{array}$ & $\begin{array}{c}\text { RFC 6956, } \\
\text { Section 5.1.1 }\end{array}$ \\
\hline 4 & EtherMACIn & $\begin{array}{l}\text { Define an Ethernet } \\
\text { input port at MAC data } \\
\text { link layer. }\end{array}$ & $\begin{array}{c}\text { RFC 6956, } \\
\text { Section 5.1.2 }\end{array}$ \\
\hline 5 & EtherClassifier & $\begin{array}{l}\text { Define the process to } \\
\text { decapsulate Ethernet } \\
\text { packets and classify } \\
\text { the packets. }\end{array}$ & $\begin{array}{c}\text { RFC 6956, } \\
\text { Section 5.1.3 }\end{array}$ \\
\hline 6 & EtherEncap & $\begin{array}{l}\text { Define the process to } \\
\text { encapsulate IP packets } \\
\text { to Ethernet packets. }\end{array}$ & $\begin{array}{c}\text { RFC 6956, } \\
\text { Section 5.1.4 }\end{array}$ \\
\hline 7 & EtherMACOut & $\begin{array}{l}\text { Define an Ethernet } \\
\text { output port at MAC } \\
\text { data link layer. }\end{array}$ & $\begin{array}{c}\text { RFC } 6956 \\
\text { Section } 5.1 .5\end{array}$ \\
\hline 8 & IPv4Validator & $\begin{array}{l}\text { Perform IPv4 packets } \\
\text { validation. }\end{array}$ & $\begin{array}{c}\text { RFC 6956, } \\
\text { Section 5.2.1 }\end{array}$ \\
\hline 9 & IPv6Validator & $\begin{array}{l}\text { Perform IPv6 packets } \\
\text { validation. }\end{array}$ & $\begin{array}{c}\text { RFC 6956, } \\
\text { Section 5.2.2 }\end{array}$ \\
\hline 10 & IPv4UcastLPM & $\begin{array}{l}\text { Perform IPv4 Longest } \\
\text { Prefix Match Lookup. }\end{array}$ & $\begin{array}{c}\text { RFC 6956, } \\
\text { Section 5.3.1 }\end{array}$ \\
\hline 11 & IPv6UcastLPM & $\begin{array}{l}\text { Perform IPv6 Longest } \\
\text { Prefix Match Lookup. }\end{array}$ & $\begin{array}{c}\text { RFC 6956, } \\
\text { Section } 5.3 .3\end{array}$ \\
\hline
\end{tabular}




\begin{tabular}{|c|c|c|c|}
\hline 12 & IPv4Next Hop & $\begin{array}{l}\text { Define the process of } \\
\text { selecting IPv4 next-hop } \\
\text { action. }\end{array}$ & $\begin{array}{c}\text { RFC 6956, } \\
\text { Section } 5.3 .2\end{array}$ \\
\hline 13 & IPv6Next Hop & $\begin{array}{l}\text { Define the process of } \\
\text { selecting IPv6 next-hop } \\
\text { action. }\end{array}$ & $\begin{array}{c}\text { RFC 6956, } \\
\text { Section } 5.3 .4\end{array}$ \\
\hline 14 & RedirectIn & $\begin{array}{l}\text { Define the process for } \\
\text { CE to inject data } \\
\text { packets into FE LFB } \\
\text { topology. }\end{array}$ & $\begin{array}{c}\text { RFC 6956, } \\
\text { Section 5.4.1 }\end{array}$ \\
\hline 15 & Redirectout & $\begin{array}{l}\text { Define the process for } \\
\text { LFBs in } \mathrm{FE} \text { to deliver } \\
\text { data packets to } \mathrm{CE} \text {. }\end{array}$ & $\begin{array}{c}\text { RFC 6956, } \\
\text { Section } 5.4 .2\end{array}$ \\
\hline 16 & $\begin{array}{c}\text { BasicMetadata } \\
\text { Dispatch }\end{array}$ & $\begin{array}{l}\text { Dispatch input packets } \\
\text { to a group output } \\
\text { according to a metadata }\end{array}$ & $\begin{array}{c}\text { RFC 6956, } \\
\text { Section 5.5.1 }\end{array}$ \\
\hline 17 & GenericScheduler & $\begin{array}{l}\text { Define a preliminary } \\
\text { generic scheduling } \\
\text { process. }\end{array}$ & $\begin{array}{c}\text { RFC } 6956, \\
\text { Section } 5.5 .2\end{array}$ \\
\hline
\end{tabular}

Table 1 


\subsection{Metadata ID}

The Metadata ID namespace is 32 bits long. Below are the guidelines for managing the namespace.

Metadata IDs in the range of 0x00000001-0x7FFFFFFF are specification Required [RFC5226]. A metadata ID using this range MUST be documented in an RFC or other permanent and readily available reference.

Values assigned by this specification:

\begin{tabular}{|c|c|c|c|c|c|}
\hline Value & Name & \multicolumn{4}{|c|}{ Definition } \\
\hline $0 \times 00000000$ & Reserved & $\mathrm{RFC}$ & 6956 & & \\
\hline $0 \times 00000001$ & PHYPort ID & $\mathrm{RFC}$ & 6956, & Section & 4.4 \\
\hline $0 \times 00000002$ & SrcMAC & $\mathrm{RFC}$ & 6956, & Section & 4.4 \\
\hline $0 \times 00000003$ & DstMAC & $\mathrm{RFC}$ & 6956, & Section & 4.4 \\
\hline $0 \times 00000004$ & LogicalportID & $\mathrm{RFC}$ & 6956, & Section & 4.4 \\
\hline $0 \times 00000005$ & EtherType & $\mathrm{RFC}$ & 6956 & Section & 4.4 \\
\hline $0 \times 00000006$ & VlanID & $\mathrm{RFC}$ & 6956, & Section & 4.4 \\
\hline $0 \times 00000007$ & VlanPriority & $\mathrm{RFC}$ & 6956, & Section & 4.4 \\
\hline $0 \times 00000008$ & Next HopIPv4Addr & $\mathrm{RFC}$ & 6956, & Section & 4.4 \\
\hline $0 \times 00000009$ & NextHopIPv6Addr & $\mathrm{RFC}$ & 6956, & Section & 4.4 \\
\hline $0 \times 0000000 \mathrm{~A}$ & Hopselector & $\mathrm{RFC}$ & 6956, & Section & 4.4 \\
\hline $0 \times 0000000 B$ & ExceptionID & $\mathrm{RFC}$ & 6956, & Section & 4.4 \\
\hline $0 \times 0000000 \mathrm{C}$ & ValidateErrorID & $\mathrm{RFC}$ & 6956, & Section & 4.4 \\
\hline $0 \times 0000000 \mathrm{D}$ & L3PortID & $\mathrm{RFC}$ & 6956, & Section & 4.4 \\
\hline $0 \times 0000000 \mathrm{E}$ & RedirectIndex & $\mathrm{RFC}$ & 6956, & Section & 4.4 \\
\hline $0 \times 0000000 \mathrm{~F}$ & MediaEncapInfoIndex & $\mathrm{RFC}$ & 6956, & Section & 4.4 \\
\hline $0 \times 80000000-$ & Reserved for & $\mathrm{RFC}$ & 6956 & & \\
\hline OXFFFFFFFF & Private Use & & & & \\
\hline
\end{tabular}

Table 2 


\subsection{Exception ID}

The Exception ID namespace is 32 bits long. Below are the guidelines for managing the namespace.

Exception IDs in the range of 0x00000000-0x7FFFFFFF are specification Required [RFC5226]. An exception ID using this range MUST be documented in an $\mathrm{RFC}$ or other permanent and readily available reference.

Values assigned by this specification:

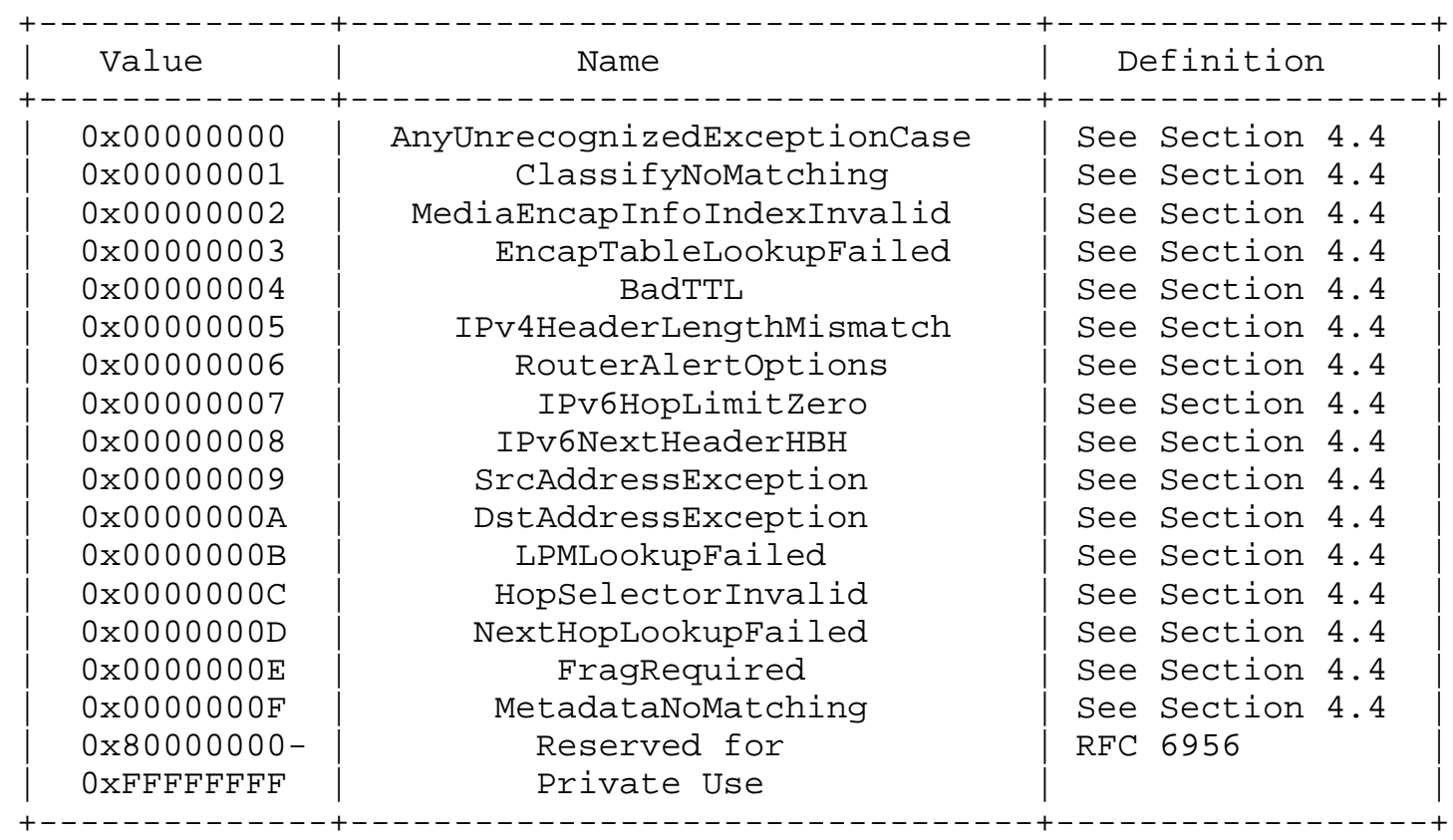

Table 3 


\subsection{Validate Error ID}

The Validate Error ID namespace is 32 bits long. Below are the guidelines for managing the namespace.

Validate Error IDs in the range of $0 \times 00000000-0 \times 7 F F F F F F F$ are Specification Required [RFC5226]. A Validate Error ID using this range MUST be documented in an RFC or other permanent and readily available reference.

Values assigned by this specification:

\begin{tabular}{|c|c|c|}
\hline Value & Name & Definition \\
\hline $0 \times 00000000$ & AnyUnrecognizedValidateErrorCase & See Section 4.4 \\
\hline $0 \times 00000001$ & InvalidIPv4PacketSize & See Section 4.4 \\
\hline $0 \times 00000002$ & Not IPv4Packet & See Section 4.4 \\
\hline $0 \times 00000003$ & InvalidIPv4HeaderLengthSize & See Section 4.4 \\
\hline $0 \times 00000004$ & InvalidIPv4LengthFieldSize & See Section 4.4 \\
\hline $0 \times 00000005$ & InvalidIPv4Checksum & See Section 4.4 \\
\hline $0 \times 00000006$ & InvalidIPv4SrcAddr & See Section 4.4 \\
\hline $0 \times 00000007$ & InvalidIPv4DstAddr & See Section 4.4 \\
\hline $0 \times 00000008$ & InvalidIPv6PacketSize & See Section 4.4 \\
\hline $0 \times 00000009$ & NotIPv6Packet & See Section 4.4 \\
\hline $0 \times 0000000 \mathrm{~A}$ & InvalidIPv6SrcAddr & See Section 4.4 \\
\hline $0 \times 0000000 B$ & InvalidIPv6DstAddr & See Section 4.4 \\
\hline $0 \times 80000000-$ & Reserved for & RFC 6956 \\
\hline $0 \times F F F F F F F$ & Private Use & \\
\hline
\end{tabular}

Table 4 


\section{Security Considerations}

The ForCES framework document [RFC3746] provides a description of the security needs for the overall ForCES architecture. For example, the ForCES protocol entities must be authenticated per the ForCES requirements before they can access the information elements described in this document via ForCES. The ForCES protocol document [RFC5810] includes a comprehensive set of security mechanisms that implementations are required to support to meet these needs. SCTPbased Transport Mapping Layer (TML) for the ForCES protocol [RFC5811] specifies security mechanisms for transport mapping for the ForCES protocol. The LFBs defined in this document are similar to other LFBs modeled by the FE model [RFC5812]. In particular, they have the same security properties. Thus, the security mechanisms and considerations from the ForCES protocol document [RFC5810] apply to this document.

10. References

10.1. Normative References

[RFC2119] Bradner, S., "Key words for use in RFCs to Indicate Requirement Levels", BCP 14, RFC 2119, March 1997.

[RFC5810] Doria, A., Hadi Salim, J., Haas, R., Khosravi, H., Wang, W., Dong, L., Gopal, R., and J. Halpern, "Forwarding and Control Element Separation (ForCES) Protocol specification", RFC 5810, March 2010.

[RFC5811] Hadi Salim, J. and K. Ogawa, "SCTP-Based Transport Mapping Layer (TML) for the Forwarding and Control Element Separation (ForCES) Protocol", RFC 5811, March 2010.

[RFC5812] Halpern, J. and J. Hadi Salim, "Forwarding and Control Element Separation (ForCES) Forwarding Element Model", RFC 5812, March 2010.

\subsection{Informative References}

[IEEE. 802-1Q] IEEE, "IEEE Standard for Local and metropolitan area networks -- Media Access Control (MAC) Bridges and Virtual Bridged Local Area Networks", IEEE Standard $802.1 Q, 2011$.

[RFC1122] Braden, R., "Requirements for Internet Hosts Communication Layers", STD 3, RFC 1122, October 1989. 
$[\mathrm{RFC} 1812]$

[RFC2460]

$[\mathrm{RFC} 2578]$

$[\mathrm{RFC} 3746]$

$[$ RFC5226]
Baker, F., "Requirements for IP Version 4 Routers", RFC 1812, June 1995.

Deering, S. and R. Hinden, "Internet Protocol, Version 6 (IPv6) Specification", RFC 2460, December 1998.

McCloghrie, K., Ed., Perkins, D., Ed., and J. Schoenwaelder, Ed., "Structure of Management Information Version 2 (SMIv2)", STD 58, RFC 2578, April 1999.

Yang, L., Dantu, R., Anderson, T., and R. Gopal, "Forwarding and Control Element Separation (ForCES) Framework", RFC 3746, April 2004.

Narten, T. and H. Alvestrand, "Guidelines for Writing an IANA Considerations section in RFCs", BCP 26, RFC 5226, May 2008. 
Appendix A. Acknowledgements

The authors would like to acknowledge the following people, whose input was particularly helpful during development of this document:

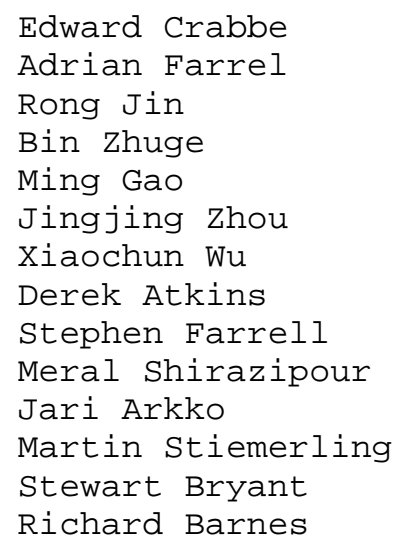

Appendix B. Contributors

The authors would like to thank Jamal Hadi Salim, Ligang Dong, and Fenggen Jia, all of whom made major contributions to the development of this document. Ligang Dong and Fenggen Jia were also two of the authors of earlier documents from which this document evolved.

Jamal Hadi Salim

Mojatatu Networks

Ottawa, Ontario

Canada

EMail: hadi@mojatatu.com

Ligang Dong

Zhejiang Gongshang University

18 Xuezheng Str., Xiasha University Town

Hangzhou 310018

P.R. China

EMail: donglg@zjsu.edu.cn

Fenggen Jia

National Digital Switching Center (NDSC)

Jianxue Road

Zhengzhou 452000

P.R. China

EMail: jfg@mail.ndsc.com.cn 


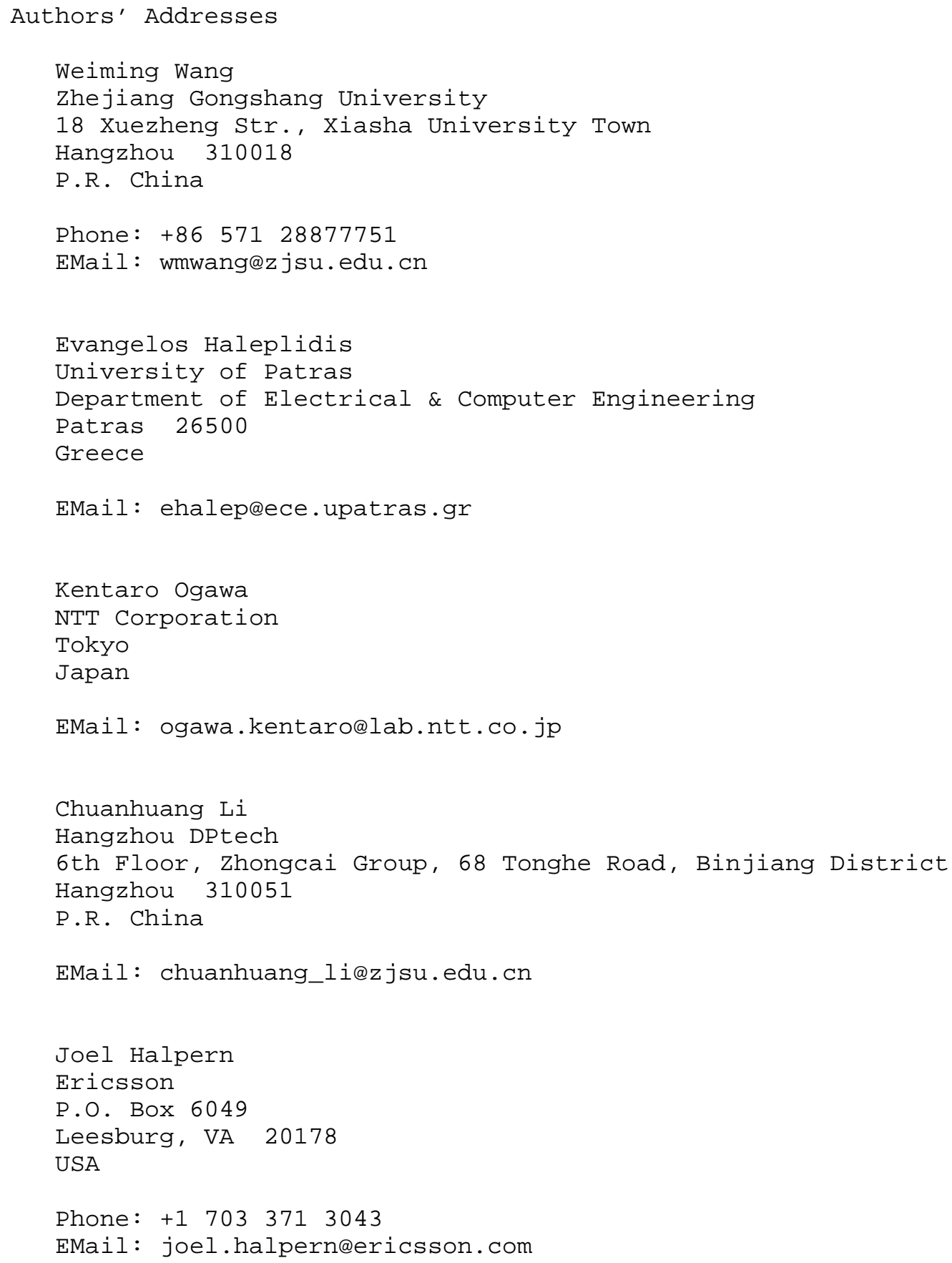

